\title{
Spitzer Follow-up of Extremely Cold Brown Dwarfs Discovered by the Backyard Worlds: Planet 9 Citizen Science Project
}

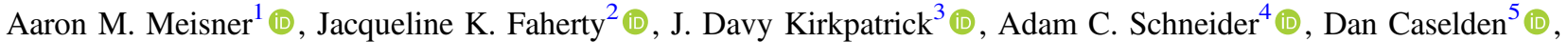

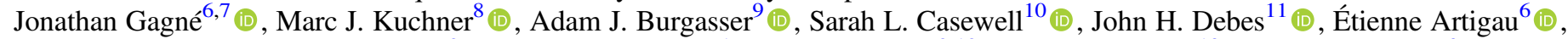 \\ Daniella C. Bardalez Gagliuffi ${ }^{2}$ (1) , Sarah E. Logsdon ${ }^{1}$ (10), Rocio Kiman ${ }^{2,12}$ (1), Katelyn Allers ${ }^{13}$ (1) , Chih-chun Hsu $^{9}$ (1), \\ John P. Wisniewski ${ }^{14}$ (D) Michaela B. Allen ${ }^{8}$, Paul Beaulieu ${ }^{15}$, Guillaume Colin ${ }^{15}$ (B), Hugo A. Durantini Luca ${ }^{16}$ (i), \\ Sam Goodman ${ }^{15}$ (D) , Léopold Gramaize ${ }^{15}$ (10), Leslie K. Hamlet ${ }^{15}$ (), Ken Hinckley ${ }^{15}$ (1) , Frank Kiwy ${ }^{15}$ (D), David W. Martin ${ }^{15}$, \\ William Pendrill ${ }^{15}$, Austin Rothermich ${ }^{17}$ (D), Arttu Sainio ${ }^{15}$ (1), Jörg Schümann ${ }^{15}$ (i), Nikolaj Stevnbak Andersen ${ }^{15}$, \\ Christopher Tanner ${ }^{15}$ (i), Vinod Thakur ${ }^{15}$, Melina Thévenot ${ }^{15}$ (i) , Jim Walla ${ }^{15}$, Zbigniew Wędracki ${ }^{15}$, Christian Aganze $^{9}$, \\ Roman Gerasimov', and Christopher Theissen ${ }^{9,18}$ \\ The Backyard Worlds: Planet 9 Collaboration \\ ${ }^{1}$ NSF’s National Optical-Infrared Astronomy Research Laboratory, 950 N. Cherry Ave., Tucson, AZ 85719, USA; ameisner@noao.edu \\ ${ }^{2}$ Department of Astrophysics, American Museum of Natural History, Central Park West at 79th St., New York, NY 10024, USA \\ ${ }^{3}$ IPAC, Mail Code 100-22, California Institute of Technology, 1200 E. California Blvd., Pasadena, CA 91125, USA \\ ${ }_{5}^{4}$ School of Earth and Space Exploration, Arizona State University, Tempe, AZ 85282, USA \\ ${ }^{5}$ Gigamon Applied Threat Research, 619 Western Ave., Suite 200, Seattle, WA 98104, USA \\ ${ }^{6}$ Institute for Research on Exoplanets, Université de Montréal, 2900 Blvd. Édouard-Montpetit Montréal, QC H3T 1J4, Canada \\ ${ }^{7}$ Planétarium Rio Tinto Alcan, Espace pour la Vie, 4801 av. Pierre-de Coubertin, Montréal, QC, Canada \\ ${ }^{8}$ NASA Goddard Space Flight Center, Exoplanets and Stellar Astrophysics Laboratory, Code 667, Greenbelt, MD 20771, USA \\ ${ }^{9}$ Center for Astrophysics and Space Science, University of California San Diego, La Jolla, CA 92093, USA \\ ${ }^{10}$ Department of Physics and Astronomy, University of Leicester, University Road, Leicester LE1 7RH, UK \\ ${ }^{11}$ ESA for AURA, Space Telescope Science Institute, 3700 San Martin Dr., Baltimore, MD 21218, USA \\ ${ }^{12}$ Department of Physics, Graduate Center, City University of New York, 365 5th Ave., New York, NY 10016, USA \\ ${ }^{13}$ Physics and Astronomy Department, Bucknell University, 701 Moore Ave., Lewisburg, PA 17837, USA \\ ${ }^{14}$ Homer L. Dodge Department of Physics and Astronomy, University of Oklahoma, 440 W. Brooks St., Norman, OK 73019, USA \\ ${ }^{15}$ Backyard Worlds: Planet 9 \\ ${ }_{17}^{16}$ IATE-OAC, Universidad Nacional de Córdoba-CONICET, Laprida 854, X5000 BGR, Córdoba, Argentina \\ ${ }^{17}$ Physics Department, University Of Central Florida, 4000 Central Florida Blvd., Orlando, FL 32816, USA \\ Received 2020 May 22; revised 2020 June 29; accepted 2020 June 30; published 2020 August 20
}

\begin{abstract}
We present Spitzer follow-up imaging of 95 candidate extremely cold brown dwarfs discovered by the Backyard Worlds: Planet 9 citizen science project, which uses visually perceived motion in multiepoch Wide-field Infrared Survey Explorer (WISE) images to identify previously unrecognized substellar neighbors to the Sun. We measure Spitzer [3.6]-[4.5] color to phototype our brown dwarf candidates, with an emphasis on pinpointing the coldest and closest Y dwarfs within our sample. The combination of WISE and Spitzer astrometry provides quantitative confirmation of the transverse motion of 75 of our discoveries. Nine of our motion-confirmed objects have best-fit linear motions larger than $1^{\prime \prime} \mathrm{yr}^{-1}$; our fastest-moving discovery is WISEA J155349.96+693355.2 $\left(\mu \approx 2\right.$ !! $\left.15 \mathrm{yr}^{-1}\right)$, a possible T-type subdwarf. We also report a newly discovered wide-separation ( $\sim 400 \mathrm{au}) \mathrm{T} 8$ comoving companion to the white dwarf LSPM J0055+5948 (the fourth such system to be found), plus a candidate late T companion to the white dwarf LSR J0002+6357 at 5.5 projected separation ( $~ 8700$ au if associated). Among our motion-confirmed targets, five have Spitzer colors most consistent with spectral type Y. Four of these five have exceptionally red Spitzer colors suggesting types of $\mathrm{Y} 1$ or later, adding considerably to the small sample of known objects in this especially valuable low-temperature regime. Our Y dwarf candidates begin bridging the gap between the bulk of the Y dwarf population and the coldest known brown dwarf.
\end{abstract}

Unified Astronomy Thesaurus concepts: Y dwarfs (1827); Brown dwarfs (185); T dwarfs (1679); Visual binary stars (1777); Proper motions (1295); Solar neighborhood (1509); Infrared astronomy (786)

Supporting material: machine-readable tables

\section{Introduction}

A complete census of the solar neighborhood provides the best way to identify and study the Galactic substellar population. The intrinsic faintness of the lowest-temperature brown dwarfs means that we can only hope to directly image those that are nearby to the Sun. For any given substellar type, the examples most amenable to detailed follow-up observations will be those that are

\footnotetext{
${ }^{18}$ NASA Sagan Fellow.
}

closest. In particular, as the James Webb Space Telescope (JWST; Gardner et al. 2006) nears launch, it is critical to identify the highest-priority brown dwarf targets for spectroscopic characterization, especially pushing into the low-mass/temperature regime of isolated exoplanet analogs.

By virtue of its unprecedented full-sky sensitivity at 3-5 $\mu \mathrm{m}$, the Wide-field Infrared Survey Explorer (WISE; Wright et al. 2010) has dramatically enhanced our ability to pinpoint the coldest brown dwarfs and reshaped our view of the solar neighborhood (e.g., 
Cushing et al. 2011; Kirkpatrick et al. 2011; Luhman 2013, $2014 \mathrm{~b})$. The WISE W1 $(3.4 \mu \mathrm{m})$ and W2 $(4.6 \mu \mathrm{m})$ bandpasses were specifically engineered to enable the selection of ultracold brown dwarfs via their red W1-W2 colors (Mainzer et al. 2011b). Including recent data from the NEOWISE mission (Mainzer et al. 2011a, 2014), WISE has now surveyed the entire sky 14 times spanning almost a decade, making identification of nearby brown dwarfs based on their large apparent motions an increasingly important tool for their discovery (e.g., Kirkpatrick et al. 2014, 2016; Luhman 2014a; Scholz 2014; Schneider et al. 2016; Kuchner et al. 2017; Marocco et al. 2019; Meisner et al. 2020).

However, the vast WISE/NEOWISE data set has yet to be exhaustively searched for cold and close brown dwarfs (e.g., Kirkpatrick et al. 2019). WISE has detected more than 2 billion unique astronomical sources (Schlafly et al. 2019), and its full imaging archive contains over 30 trillion pixels. Despite modern computing resources, human vetting still plays an integral role in the discovery of moving objects among a sea of interlopers and artifacts (e.g., Kirkpatrick et al. 2014, 2016; Schneider et al. 2016).

To overcome the bottleneck of visual inspection encountered by WISE-based moving object searches, we initiated the Backyard Worlds: Planet 9 citizen science project (http:// backyardworlds.org; Kuchner et al. 2017, hereafter Backyard Worlds). Backyard Worlds crowdsources the visual vetting workload among thousands of volunteers who participate via the internet, viewing animated blinks of WISE images spanning the full WISE+NEOWISE time baseline. In this work we present a sample of 96 candidate extremely cold brown dwarfs discovered by Backyard Worlds volunteers. For this sample, which generally lacks high-significance detections at W1, we have obtained critical Spitzer/IRAC (Werner et al. 2004; Fazio et al. 2004) photometry at $3.6 \mu \mathrm{m}$ (ch1) and $4.5 \mu \mathrm{m}$ (ch2). Secure ch1 detections enable estimates of key parameters, including spectral type, effective temperature, and distance.

In Section 2 we briefly summarize the WISE and NEOWISE missions. In Section 3 we provide background information about the Backyard Worlds project. In Section 4 we explain our selection of the Spitzer follow-up targets that compose our sample (Section 5). In Section 6 we discuss our Spitzer observing strategy. In Section 7 we present our Spitzer photometry. In Section 8 we present follow-up spectroscopic confirmations. In Section 9 we perform WISE and Spitzer astrometry in order to assess significance of motion. In Section 10 we present follow-up and archival near-infrared (NIR) photometry for our sample. In Section 11 we synthesize our photometry, spectroscopy, and astrometry results, commenting on various individual objects of particular interest. We conclude in Section 12.

\section{WISE/NEOWISE Overview}

WISE is a $0.4 \mathrm{~m}$ aperture space telescope in low Earth orbit, launched in late 2009. WISE mapped the entire sky once in all four of its broad mid-infrared channels (W1 $=3.4 \mu \mathrm{m}$, $\mathrm{W} 2=4.6 \mu \mathrm{m}, \mathrm{W} 3=12 \mu \mathrm{m}$, and $\mathrm{W} 4=22 \mu \mathrm{m})$ during the first half of 2010 (Cutri et al. 2012). In the latter part of 2010 the two longest wavelength bands became unusable owing to exhaustion of onboard cryogen. However, WISE continued to operate in W1 and W2 through early 2011 thanks to the NEOWISE mission extension (Mainzer et al. 2011a). WISE was put into hibernation from 2011 February until 2013 December, at which point it recommenced surveying in $\mathrm{W} 1$ and $\mathrm{W} 2$ as part of the NEOWISE-Reactivation mission (NEOWISE-R; Mainzer et al. 2014; Cutri et al. 2015). NEOWISE-R has continued its W1/W2 observations ever since.

A typical sky location is observed for a $\sim 1$-day time period once every 6 months. WISE has now completed a total of 14 such full-sky mappings in W1 and W2. By co-adding exposures within each 6-monthly visit to each sky location, it is possible to construct a time series of deep and clean WISE co-adds optimized for detecting faint moving objects (Meisner et al. 2018b, 2018c, 2019) while leveraging a time baseline $>10 \times$ longer than that of AllWISE (Cutri et al. 2013). The joint analysis of WISE and NEOWISE data therefore opens up a huge discovery space for finding faint moving objects, such as cold brown dwarfs, in the mid-infrared.

\section{Backyard Worlds Overview}

In order to fully mine the combined WISE+NEOWISE data set for moving object discoveries, we launched the Backyard Worlds: Planet 9 citizen science project on 2017 February 15 (Kuchner et al. 2017). Backyard Worlds crowdsources the process of visually confirming motion in WISE images by distributing animated "flipbooks" via the Zooniverse web portal (Simpson et al. 2014). Each flipbook shows a time-series blink covering a random ${ }^{19} \sim 10^{\prime} \times 10^{\prime}$ patch of sky. In detail, each frame of each blink is a color-composite difference image meant to null out static background sources. Each difference image is built by creating one co-added sky pass worth of WISE data (a "time-resolved unWISE co-add"; Meisner et al. 2018b) and then subtracting off a static sky template built by co-adding other epochs of WISE data. Very fast moving objects (motions of many arcseconds per year or larger) will appear unsubtracted, while slower-moving sources (down to a few hundred mas $\mathrm{yr}^{-1}$ ) will manifest as partially subtracted "dipoles." The blue (red) channel represents W1 (W2), so that redder (i.e., colder) moving objects will appear distinctively orange. ${ }^{20}$

Although Backyard Worlds places substantial emphasis on discovering very cold and close $\mathrm{Y}$ dwarfs, participants are encouraged to report any moving object not presented in the prior literature (i.e., not in the SIMBAD database; Wenger et al. 2000). Examples include comoving substellar companions to higher-mass primaries (e.g., Faherty et al. 2020), white dwarfs with infrared excesses (e.g., Debes et al. 2019), and hypothesized planets in the outer solar system (e.g., Matese et al. 1999; Matese \& Whitmire 2011; Luhman 2014a; Batygin \& Brown 2016; Meisner et al. 2017, 2018a).

As of 2020 April, Backyard Worlds counts more than 58,000 registered users, with the number of unique participants (including those not registered) estimated to be several times larger. Among these participants are 200 advanced users who collaborate closely with one another and the science team. Advanced users have created a number of custom motion search tools that expand the Backyard Worlds effort significantly beyond its presence on the Zooniverse web portal (e.g., WiseView; Caselden et al. 2018) and often make use of catalog

\footnotetext{
19 The locations are truly random; there is no preselection of the flipbook footprints to, e.g., be centered on a suspected brown dwarf candidate.

20 The Backyard Worlds documentation at https://www.zooniverse.org/ projects/marckuchner/backyard-worlds-planet-9/about/research contains example images illustrating this color scheme and the appearance of dipoles and fast movers in our flipbooks.
} 
querying interfaces such as SIMBAD, IRSA, Vizier (Ochsenbein et al. 2000), and the Astro Data Lab (Fitzpatrick et al. 2014). See Figure 1 of Kuchner et al. (2017) for an example Backyard Worlds flipbook image and Figure 1 of Meisner et al. (2020) for an example WiseView image sequence.

\section{Spitzer Target Selection}

Our Spitzer follow-up consists of two separate observing campaigns, programs 14076 and 14299, which we will refer to as p14076 and p14299 (PI: Faherty in both cases). Target selection for both campaigns was essentially the same, though performed at different times: p14076 (p14299) targets were selected in 2018 March (2019 April).

Backyard Worlds maintains a running list of all usersubmitted, previously unpublished candidate moving objects with motions that have been visually confirmed by our science team and archival photometry suggesting L, T, or Y spectral types. This list currently contains $\sim 1600$ newly discovered brown dwarf candidates and represents the parent sample from which we selected our Spitzer photometry targets. At the time of p14076 (p14299) target selection, this parent sample of Backyard Worlds discoveries included $\sim 800(\sim 1200)$ brown dwarf candidates.

The primary goal of our Spitzer photometry campaigns was to pinpoint the strongest $\mathrm{Y}$ dwarf candidates among our moving object discoveries. As such, the majority of our targets were selected because they bear the hallmarks of potential $\mathrm{Y}$ dwarfs: detected only in W2 (undetected in W1 and other optical/infrared surveys) and exhibiting clear motion consistent with that of a brown dwarf in the solar neighborhood. Additionally, we selected some Spitzer targets that have a faint W1 counterpart visible if any of the following three conditions were met: the crude phototype based on $\mathrm{W} 1-\mathrm{W} 2$ color suggested possible membership within the $20 \mathrm{pc}$ sample, the candidate appeared to be a potential common proper motion (CPM) companion to another moving object, and the motion was exceptionally large $\left(\mu \gtrsim 1^{\prime \prime} \mathrm{yr}^{-1}\right)$. With these selection rules in mind, we reexamined our full list of Backyard Worlds discoveries prior to each Spitzer campaign's proposal submission and requested observations for those objects that matched our criteria for meriting Spitzer follow-up. One major driver behind our p14299 effort was the desire to phototype relatively recent Backyard Worlds discoveries before the impending retirement of Spitzer.

We do not expect our brown dwarf target list to be contaminated by W2-only solar system objects. Because their apparent motions are so large, inner solar system objects are entirely nulled out by our co-addition of WISE data into daily intervals. At Kuiper Belt distances, faint solar system objects should also be largely removed by our stacking, and their degree-scale parallaxes easily distinguish them from brown dwarfs in the solar neighborhood. At distances of order $1000 \mathrm{au}$ (e.g., Batygin \& Brown 2016) a planetary body would remain present in our flipbooks, but the corresponding parallax of several arcminutes would again allow us to separate such a candidate from our brown dwarf targets. For parallaxes of $\sim 1^{\prime}$ to $\sim 1^{\circ}$, the WISE data would appear to show one solar system body as a pair of linear tracklets at sky locations offset by roughly twice the parallax. Each of these two apparitions would be perceived during our target vetting visual inspections (performed on isolated arcminute-sized cutouts) as "vanishing" during every other sky pass, which is inconsistent with the signature of a brown dwarf in the solar neighborhood. Therefore, our Spitzer brown dwarf target sample will exclude objects at a few thousand au or closer even though we have not attempted to perform solar system orbit linking on arcminute or degree angular scales. During our brown dwarf target selection process, sources alternating in sky position by a few arcminutes or more from one sky pass to the next would generally have been discarded and presumed to be "latent" detector artifacts, which were found to be a common contaminant in the WISE Planet 9 search of Meisner et al. (2017). At distances of 10,000-30,000 au (e.g., Matese \& Whitmire 2011), the trajectory of a solar companion would be dominated by many arcseconds of oscillatory parallactic motion, but none of our Spitzer targets display such behavior.

We ultimately selected 65 targets for p14076 and 33 targets for p14299. p14299 inadvertently retargeted two objects from p14076 (WISEA 0651-8355 and WISEA 1627-2443), meaning that our full Spitzer follow-up sample contains 96 unique sources. The sample presented in this work additionally includes one object (CWISE 0002+6352) for which we have analyzed serendipitous archival Spitzer imaging from the GLIMPSE360 program (Churchwell et al. 2009).

During p14299 target selection, we sought to avoid duplicating any objects already slated for Spitzer observations by the CatWISE team (Spitzer program 14034, p14034 for short; Eisenhardt et al. 2020; Meisner et al. 2020). The Backyard Worlds and CatWISE moving object discovery lists overlap extensively, since both projects seek to uncover faint brown dwarfs using motions spanning the combined WISE + NEOWISE time baseline.

\section{Spitzer Target Sample Properties}

In our estimation, the combined p14076+p14299 sample is best viewed as effectively AllWISE selected, resulting from a combination of numerous AllWISE queries issued by Backyard Worlds advanced users. A total of 91\% (87/96) of our discoveries are present in the AllWISE catalog, despite the fact that the full WISE+NEOWISE data set now contains $>2 \times$ more securely detected sources than AllWISE (Schlafly et al. 2019). The discoveries of four of our nine targets not in AllWISE (WISEU 0019-0943, WISEU 0055+5847, WISEU $0505+3043$, WISEU $2150-7520$ ) are readily explained by dedicated CPM searches where WISE visual inspection was seeded by the Gaia DR2 (Gaia Collaboration et al. 2016, 2018) catalog of higher-mass objects in the solar neighborhood.

Because our Spitzer target selection was finalized before the public release of CatWISE (Eisenhardt et al. 2020) and we believe our sample to be largely AllWISE selected, we adopt AllWISE designations for our discoveries when available. In the absence of an AllWISE counterpart, we then use the CatWISE designation if one is available, and we finally employ unWISE Catalog designations when neither AllWISE nor CatWISE contains a counterpart.

Our sample's spatial distribution in Galactic coordinates is shown in Figure 1. As expected, our brown dwarf candidates are scattered across the entire sky, with a significant underdensity in the crowded Galactic plane-only 6\% (6/97) of objects inhabit the $\left|b_{\text {gal }}\right|<10^{\circ}$ sky region that accounts for $17 \%$ of the sky. There also appears to be somewhat of an overdensity toward the south Galactic cap. 


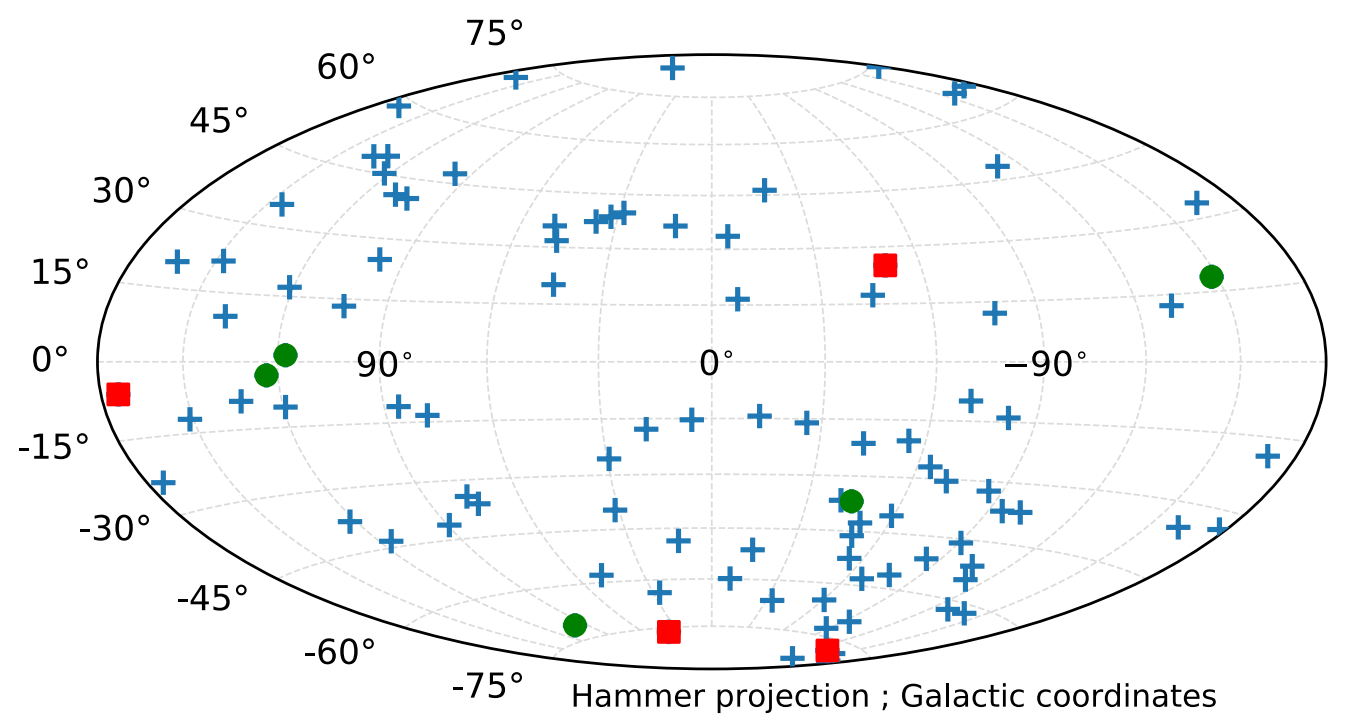

Figure 1. Full-sky distribution of all 96 Backyard Worlds targets followed up with Spitzer programs 14076 and 14299, plus one discovery (CWISE 0002+6352) with archival Spitzer data, in Galactic Hammer projection. CPM candidates are denoted by green circles. Red squares are spurious candidates (Table 1) for which no Spitzer counterpart was found. The locations of all other brown dwarf candidates are shown as blue plus signs.

\subsection{Targets Previously Presented by Backyard Worlds}

The discoveries of six of our targets have been previously published by our team: WISEU $2150-7520$ in Faherty et al. (2020) plus WISEA 0014+7951, WISEA 0830+2837, WISEA 0830-6323, WISEA $1516+7217$, and WISEA $1525+6053$ in Bardalez Gagliuffi et al. (2020). These prior publications should be considered the definitive references for WISEU 2150 -7520 and WISEA $0830+2837$, as they contain more detailed treatments of these objects. For WISEA 0014+7951, WISEA 0830-6323, WISEA 1516+7217, and WISEA 1525+6053, the present work provides significant new information by motion-confirming these targets and supplying more accurate WISE+Spitzer proper-motion measurements for them.

\subsection{Overlap with Previously Published CatWISE Spitzer Follow-up}

Although we intended to avoid duplicating any Spitzer follow-up of brown dwarf discoveries already published by the CatWISE team (Marocco et al. 2019, 2020; Meisner et al. 2020), we inadvertently targeted CWISEP 1359-4352 in p14299. We nevertheless propagate this object through our entire analysis pipeline in this work, since its p14299 Spitzer photometry is deeper than the p14034 photometry initially presented in Meisner et al. (2020).

\subsection{Spurious Candidates}

Four brown dwarf candidates were found to have no Spitzer counterpart (see Table 1 and Section 7). These spurious candidates are thought to be very faint noise excursions or artifacts in the WISE data. In addition to the targets in Table 1, WISEA J162716.41-244355.4 clearly has an extended morphology based on our Spitzer imaging and is therefore presumed to be a piece of nebulosity in the $\rho$ Ophiuchi molecular cloud complex rather than a moving object.

\subsection{WISE Photometry}

Table 2 provides WISE photometry for our entire sample of 96 targets. In gathering WISE photometry, we gave preference
Table 1

Targets without Any Spitzer Counterpart

\begin{tabular}{lcc}
\hline \hline AOR & Designation & $\begin{array}{c}\text { W2 } \\
(\mathrm{mag})\end{array}$ \\
\hline 70080512 & WISEA J014614.70-290048.4 & $16.49 \pm 0.20$ \\
70082560 & WISEU J050511.06+304355.6 & $16.38 \pm 0.12$ \\
70084096 & WISEA J132441.18-372024.8 & $16.25 \pm 0.19$ \\
70083072 & WISEA J235136.37-250247.6 & $16.14 \pm 0.19$ \\
\hline
\end{tabular}

Note. Each object's designation prefix corresponds to the WISE catalog from which the quoted W2 magnitude has been drawn.

(This table is available in machine-readable form.)

to CatWISE photometry when available, since CatWISE fluxes are fit to linearly moving profiles that account for source motion and CatWISE incorporates $4 \times$ more input W1/W2 imaging than AllWISE. In the absence of CatWISE photometry, we next checked AllWISE for photometry. In the event that neither CatWISE nor AllWISE photometry was available, we use photometry from the unWISE Catalog (Schlafly et al. 2019). Note that the unWISE Catalog performs source detection and photometry independently in W1 and W2, and so a few objects in Table 2 have W2 photometry from the unWISE Catalog but no corresponding W1 photometry. Also note that in general the identifier prefixes we have chosen (typically WISEA for AllWISE) do not correspond to the origin of the WISE photometry listed in Table 2 (typically CatWISE). For WISEU 2150-7520 we report the custom WISE photometry from Faherty et al. (2020). Table 2 lists the origin of WISE photometry reported for each target. All magnitudes quoted throughout this paper are in the Vega system unless otherwise noted.

Figure 2 shows a histogram of the W2 magnitudes of the 96 new discoveries in our sample. The median W2 magnitude of this sample is $\mathrm{W} 2=15.70 \mathrm{mag}$. This is much fainter than all prior full-sky WISE motion searches except for Meisner et al. (2020), which had a median W2 magnitude of W2 $=15.93$ mag. Both Backyard Worlds and CatWISE have been able to push much fainter than previous full-sky WISE motion 
Table 2

Mid-infrared Photometry from WISE and Spitzer

\begin{tabular}{|c|c|c|c|c|c|c|c|c|c|}
\hline Name & $\begin{array}{c}\text { Motion } \\
\text { Confirmed }\end{array}$ & $\begin{array}{l}\text { W1 } \\
\text { (mag) }\end{array}$ & $\begin{array}{l}\text { W2 } \\
\text { (mag) }\end{array}$ & $\begin{array}{l}\text { W1-W2 } \\
(\mathrm{mag})\end{array}$ & AOR & $\begin{array}{l}\text { ch1 } \\
\text { (mag) }\end{array}$ & $\begin{array}{c}\mathrm{ch} 2 \\
(\mathrm{mag})\end{array}$ & $\begin{array}{l}\text { ch1-ch2 } \\
(\mathrm{mag})\end{array}$ & $\begin{array}{c}\text { WISE } \\
\text { Phot. Reference }\end{array}$ \\
\hline CWISE J000229.93+635217.0 & yes & $18.21 \pm 0.21$ & $15.73 \pm 0.05^{\mathrm{b}}$ & $2.48 \pm 0.22$ & 38724864 & $17.349 \pm 0.246$ & $15.690 \pm 0.055$ & $1.659 \pm 0.252$ & 6 \\
\hline WISEA J001449.96+795116.1 & yes & $18.72 \pm 0.28$ & $16.00 \pm 0.06$ & $2.72 \pm 0.28$ & 65969152 & $17.752 \pm 0.059$ & $15.863 \pm 0.019$ & $1.890 \pm 0.062$ & 1 \\
\hline WISEU J001908.31-094323.3 & yes & $18.33 \pm 0.17$ & $15.81 \pm 0.07^{\mathrm{b}}$ & $2.52 \pm 0.19$ & 70084352 & $17.119 \pm 0.034$ & $15.666 \pm 0.019$ & $1.453 \pm 0.038$ & 2 \\
\hline WISEA J002810.59+521853.1 & yes & $18.05 \pm 0.13$ & $15.60 \pm 0.05$ & $2.45 \pm 0.14$ & 68143872 & $17.360 \pm 0.043$ & $15.669 \pm 0.019$ & $1.691 \pm 0.046$ & 1 \\
\hline WISEA J002909.90+190516.6 & yes & $17.58 \pm 0.10$ & $15.36 \pm 0.05$ & $2.21 \pm 0.11$ & 65969664 & $16.727 \pm 0.025$ & $15.301 \pm 0.018$ & $1.426 \pm 0.032$ & 1 \\
\hline WISEA J003631.29-642735.6 & no & $18.31 \pm 0.16$ & $15.86 \pm 0.05$ & $2.45 \pm 0.17$ & 70079744 & $17.203 \pm 0.037$ & $15.816 \pm 0.019$ & $1.388 \pm 0.041$ & 1 \\
\hline WISEU J004851.21+250814.9 & yes & $\ldots$ & $15.04 \pm 0.03^{\mathrm{b}}$ & $\ldots$ & 68141824 & $16.793 \pm 0.026$ & $14.743 \pm 0.016$ & $2.050 \pm 0.031$ & 2 \\
\hline WISEU J005559.88+594745.0 & yes & $\ldots$ & $15.20 \pm 0.03^{\mathrm{b}}$ & $\ldots$ & 68141568 & $16.450 \pm 0.021$ & $14.965 \pm 0.016$ & $1.485 \pm 0.026$ & 2 \\
\hline WISEA J010116.12-520629.8 & yes & $18.85 \pm 0.26$ & $15.88 \pm 0.05$ & $2.98 \pm 0.27$ & 65970432 & $17.687 \pm 0.056$ & $15.692 \pm 0.019$ & $1.994 \pm 0.060$ & 1 \\
\hline WISEA J012057.06-764216.4 & yes & $18.87 \pm 0.21$ & $15.87 \pm 0.05$ & $2.99 \pm 0.22$ & 65970944 & $17.663 \pm 0.056$ & $15.790 \pm 0.019$ & $1.874 \pm 0.059$ & 1 \\
\hline WISEA J012834.87-280302.5 & yes & $17.47 \pm 0.09$ & $15.55 \pm 0.05$ & $1.92 \pm 0.10$ & 70085632 & $16.722 \pm 0.025$ & $15.511 \pm 0.018$ & $1.211 \pm 0.032$ & 1 \\
\hline WISEA J013217.78-581825.9 & yes & $19.10 \pm 0.43$ & $15.72 \pm 0.05$ & $3.38 \pm 0.43$ & 65971200 & $17.882 \pm 0.064$ & $15.740 \pm 0.019$ & $2.141 \pm 0.067$ & 1 \\
\hline WISEU J013522.46-221957.3 & yes & $\ldots$ & $15.85 \pm 0.07^{\mathrm{b}}$ & $\ldots$ & 70082048 & $17.154 \pm 0.035$ & $15.793 \pm 0.019$ & $1.362 \pm 0.039$ & 2 \\
\hline WISEA J013810.78-412220.2 & yes & $18.40 \pm 0.17$ & $16.32 \pm 0.09$ & $2.08 \pm 0.19$ & 68143616 & $17.434 \pm 0.044$ & $16.198 \pm 0.021$ & $1.236 \pm 0.049$ & 1 \\
\hline WISEA J014603.23-261908.7 & yes & $>18.88$ & $15.92 \pm 0.07$ & $>2.96$ & 68142080 & $17.490 \pm 0.047$ & $15.810 \pm 0.019$ & $1.680 \pm 0.052$ & 1 \\
\hline WISEA J015507.35-421635.7 & yes & $18.91 \pm 0.26$ & $16.23 \pm 0.08$ & $2.68 \pm 0.27$ & 70079488 & $18.375 \pm 0.100$ & $16.165 \pm 0.020$ & $2.210 \pm 0.102$ & 1 \\
\hline WISEA J015601.22+525304.4 & no & $17.26 \pm 0.07$ & $15.43 \pm 0.04$ & $1.83 \pm 0.08$ & 70082816 & $16.697 \pm 0.024$ & $15.354 \pm 0.018$ & $1.343 \pm 0.030$ & 1 \\
\hline WISEA J021420.18-573245.1 & yes & $18.94 \pm 0.24$ & $16.25 \pm 0.07$ & $2.69 \pm 0.25$ & 65971712 & $18.075 \pm 0.079$ & $16.157 \pm 0.020$ & $1.918 \pm 0.081$ & 1 \\
\hline WISEA J025756.40-265528.8 & yes & $18.70 \pm 0.33$ & $16.20 \pm 0.09$ & $2.50 \pm 0.34$ & 68144384 & $18.302 \pm 0.097$ & $15.953 \pm 0.019$ & $2.348 \pm 0.099$ & 1 \\
\hline WISEA J025805.29-321917.4 & yes & $16.56 \pm 0.04$ & $14.91 \pm 0.03^{\mathrm{b}}$ & $1.65 \pm 0.05$ & 70084608 & $16.025 \pm 0.016$ & $14.988 \pm 0.016$ & $1.037 \pm 0.023$ & 1 \\
\hline WISEA J030534.09-582635.5 & yes & $18.29 \pm 0.12$ & $15.55 \pm 0.03$ & $2.74 \pm 0.13$ & 65971968 & $17.340 \pm 0.042$ & $15.515 \pm 0.018$ & $1.824 \pm 0.046$ & 1 \\
\hline WISEA J032600.28+421056.8 & yes & $>19.03$ & $15.28 \pm 0.04$ & $>3.75$ & 65972224 & $17.230 \pm 0.038$ & $15.292 \pm 0.018$ & $1.938 \pm 0.042$ & 1 \\
\hline WISEA J032811.00-422321.6 & yes & $18.56 \pm 0.25$ & $16.29 \pm 0.07$ & $2.27 \pm 0.26$ & 65972480 & $17.930 \pm 0.066$ & $16.026 \pm 0.020$ & $1.904 \pm 0.069$ & 1 \\
\hline WISEA J034253.20+673143.5 & no & $17.69 \pm 0.10$ & $15.56 \pm 0.05$ & $2.12 \pm 0.11$ & 70085888 & $16.488 \pm 0.021$ & $15.411 \pm 0.018$ & $1.077 \pm 0.028$ & 1 \\
\hline WISEA J034227.44-462252.0 & yes & $18.89 \pm 0.32$ & $16.12 \pm 0.06$ & $2.77 \pm 0.32$ & 70086144 & $17.714 \pm 0.057$ & $16.132 \pm 0.021$ & $1.582 \pm 0.060$ & 1 \\
\hline WISEA J035410.03-572104.0 & no & $18.57 \pm 0.23$ & $16.10 \pm 0.08$ & $2.47 \pm 0.24$ & 70085376 & $17.773 \pm 0.061$ & $16.059 \pm 0.020$ & $1.714 \pm 0.064$ & 1 \\
\hline WISEA J035733.85+070557.4 & yes & $17.78 \pm 0.14$ & $15.37 \pm 0.05$ & $2.42 \pm 0.14$ & 65972736 & $16.736 \pm 0.026$ & $15.401 \pm 0.018$ & $1.335 \pm 0.032$ & 1 \\
\hline WISEA J040702.42+190945.8 & yes & $17.55 \pm 0.11$ & $15.55 \pm 0.06$ & $2.01 \pm 0.13$ & 65972992 & $16.837 \pm 0.027$ & $15.444 \pm 0.018$ & $1.392 \pm 0.033$ & 1 \\
\hline WISEA J042236.95-044203.5 & yes & $18.82 \pm 0.41$ & $16.02 \pm 0.08^{\mathrm{b}}$ & $2.80 \pm 0.41$ & 68140544 & $17.694 \pm 0.054$ & $15.937 \pm 0.020$ & $1.758 \pm 0.058$ & 1 \\
\hline WISEA J050238.28+100750.0 & yes & $18.91 \pm 0.51$ & $15.35 \pm 0.05$ & $3.56 \pm 0.51$ & 65973760 & $17.520 \pm 0.047$ & $15.363 \pm 0.018$ & $2.156 \pm 0.050$ & 1 \\
\hline WISEU J050305.68-564834.0 & yes & $\ldots$ & $16.71 \pm 0.09^{\mathrm{b}}$ & $\ldots$ & 68142592 & $18.997 \pm 0.185$ & $16.022 \pm 0.020$ & $2.975 \pm 0.185$ & 2 \\
\hline WISEA J050615.56-514521.3 & yes & $18.49 \pm 0.14$ & $16.71 \pm 0.10$ & $1.78 \pm 0.17$ & 70013696 & $18.340 \pm 0.098$ & $16.233 \pm 0.021$ & $2.107 \pm 0.100$ & 1 \\
\hline WISEA J053535.43-644518.5 & yes & $15.26 \pm 0.04$ & $15.08 \pm 0.03^{\mathrm{b}}$ & $0.17 \pm 0.04$ & 70083584 & $15.089 \pm 0.012$ & $15.087 \pm 0.016$ & $0.002 \pm 0.020$ & 1 \\
\hline WISEA J053512.01-773829.7 & yes & $>19.58$ & $15.90 \pm 0.05$ & $>3.68$ & 68142848 & $18.120 \pm 0.082$ & $15.881 \pm 0.019$ & $2.240 \pm 0.084$ & 1 \\
\hline WISEA J061713.07+670400.8 & no & $18.22 \pm 0.15$ & $15.89 \pm 0.06$ & $2.32 \pm 0.16$ & 70080000 & $17.876 \pm 0.065$ & $15.972 \pm 0.019$ & $1.905 \pm 0.068$ & 1 \\
\hline WISEA J064503.72+524054.1 & yes & $16.85 \pm 0.05$ & $15.09 \pm 0.03^{\mathrm{b}}$ & $1.75 \pm 0.06$ & 68144128 & $17.157 \pm 0.035$ & $15.138 \pm 0.017$ & $2.019 \pm 0.039$ & 4 \\
\hline WISEA J065113.90-835502.6 & yes & $18.29 \pm 0.11$ & $15.61 \pm 0.04$ & $2.68 \pm 0.12$ & 70013952 & $17.424 \pm 0.043$ & $15.534 \pm 0.018$ & $1.889 \pm 0.047$ & 1 \\
\hline WISEA J075438.20+090044.9 & yes & $18.48 \pm 0.24$ & $15.78 \pm 0.08$ & $2.71 \pm 0.25$ & 65974528 & $17.584 \pm 0.049$ & $15.741 \pm 0.019$ & $1.844 \pm 0.052$ & 1 \\
\hline WISEA J080622.22-082046.5 & yes & $>18.79$ & $15.49 \pm 0.12$ & $>3.31$ & 65974784 & $17.295 \pm 0.041$ & $15.411 \pm 0.018$ & $1.884 \pm 0.045$ & 3 \\
\hline WISEA J083011.95+283716.0 & yes & $>18.83$ & $15.84 \pm 0.14$ & $>2.99$ & 65975296 & $19.100 \pm 0.199$ & $15.845 \pm 0.019$ & $3.254 \pm 0.200$ & 3 \\
\hline WISEA J083019.97-632305.4 & yes & $18.12 \pm 0.10$ & $15.87 \pm 0.04$ & $2.25 \pm 0.10$ & 65975040 & $17.497 \pm 0.049$ & $15.675 \pm 0.018$ & $1.821 \pm 0.052$ & 1 \\
\hline WISEA J084329.01+694709.8 & yes & $18.23 \pm 0.16$ & $16.21 \pm 0.08$ & $2.03 \pm 0.18$ & 70081792 & $17.213 \pm 0.036$ & $16.013 \pm 0.020$ & $1.201 \pm 0.042$ & 1 \\
\hline WISEA J101804.20-684254.0 & yes & $17.41 \pm 0.06$ & $15.54 \pm 0.03^{\mathrm{b}}$ & $1.88 \pm 0.07$ & 65975552 & $17.324 \pm 0.043$ & $15.406 \pm 0.017$ & $1.918 \pm 0.046$ & 1 \\
\hline WISEA J104051.77+450329.3 & no & $17.89 \pm 0.12$ & $15.22 \pm 0.04$ & $2.67 \pm 0.13$ & 68143104 & $16.949 \pm 0.030$ & $15.285 \pm 0.018$ & $1.664 \pm 0.034$ & 1 \\
\hline
\end{tabular}


Table 2

(Continued)

\begin{tabular}{|c|c|c|c|c|c|c|c|c|c|}
\hline Name & $\begin{array}{l}\text { Motion } \\
\text { Confirmed }\end{array}$ & $\begin{array}{l}\text { W1 } \\
\text { (mag) }\end{array}$ & $\begin{array}{l}\mathrm{W} 2 \\
\text { (mag) }\end{array}$ & $\begin{array}{c}\mathrm{W} 1-\mathrm{W} 2 \\
(\mathrm{mag})\end{array}$ & AOR & $\begin{array}{l}\text { ch1 } \\
(\mathrm{mag})\end{array}$ & $\begin{array}{c}\mathrm{ch} 2 \\
(\mathrm{mag})\end{array}$ & $\begin{array}{l}\mathrm{ch} 1-\mathrm{ch} 2 \\
(\mathrm{mag})\end{array}$ & $\begin{array}{c}\text { WISE } \\
\text { Phot. Reference }^{c}\end{array}$ \\
\hline WISEA J104216.89-003935.9 & yes & $18.07 \pm 0.17$ & $15.62 \pm 0.05$ & $2.45 \pm 0.18$ & 65975808 & $17.368 \pm 0.043$ & $15.745 \pm 0.019$ & $1.624 \pm 0.047$ & 1 \\
\hline WISEA J105349.41-460241.2 & yes & $17.18 \pm 0.07$ & $15.27 \pm 0.04^{\mathrm{b}}$ & $1.91 \pm 0.08$ & 65976064 & $17.159 \pm 0.035$ & $15.258 \pm 0.017$ & $1.901 \pm 0.039$ & 1 \\
\hline WISEA J105917.38+285729.3 & yes & $18.06 \pm 0.16$ & $15.60 \pm 0.05$ & $2.46 \pm 0.16$ & 70083840 & $17.295 \pm 0.039$ & $15.593 \pm 0.019$ & $1.702 \pm 0.043$ & 1 \\
\hline WISEA J110201.76+350335.4 & yes & $18.32 \pm 0.27$ & $15.18 \pm 0.04$ & $3.14 \pm 0.27$ & 65976320 & $17.278 \pm 0.038$ & $15.132 \pm 0.017$ & $2.147 \pm 0.043$ & 1 \\
\hline WISEA J112440.19+663052.0 & yes & $18.11 \pm 0.13$ & $15.40 \pm 0.04$ & $2.71 \pm 0.13$ & 70016512 & $17.162 \pm 0.035$ & $15.319 \pm 0.017$ & $1.844 \pm 0.039$ & 1 \\
\hline WISEA J114350.90+401333.9 & yes & $18.45 \pm 0.28$ & $15.59 \pm 0.05$ & $2.86 \pm 0.29$ & 65977344 & $17.397 \pm 0.042$ & $15.486 \pm 0.018$ & $1.911 \pm 0.046$ & 1 \\
\hline WISEA J114601.22+342458.8 & yes & $18.08 \pm 0.14$ & $15.62 \pm 0.06$ & $2.46 \pm 0.15$ & 65977600 & $16.899 \pm 0.029$ & $15.447 \pm 0.018$ & $1.452 \pm 0.034$ & 1 \\
\hline WISEA J115917.89+671704.2 & yes & $19.20 \pm 0.43$ & $16.14 \pm 0.07$ & $3.06 \pm 0.44$ & 65977856 & $18.129 \pm 0.081$ & $15.951 \pm 0.019$ & $2.178 \pm 0.083$ & 1 \\
\hline WISEA J125721.01+715349.3 & yes & $19.01 \pm 0.24$ & $16.11 \pm 0.06$ & $2.90 \pm 0.24$ & 65978112 & $18.889 \pm 0.162$ & $16.158 \pm 0.021$ & $2.731 \pm 0.163$ & 1 \\
\hline WISEA J131102.03+312107.9 & no & $19.11 \pm 0.47$ & $15.76 \pm 0.06$ & $3.35 \pm 0.48$ & 70085120 & $17.587 \pm 0.049$ & $15.741 \pm 0.019$ & $1.846 \pm 0.052$ & 1 \\
\hline WISEA J135042.33-830202.8 & no & $19.30 \pm 0.49$ & $16.78 \pm 0.19$ & $2.52 \pm 0.52$ & 70084864 & $17.728 \pm 0.056$ & $16.785 \pm 0.026$ & $0.943 \pm 0.062$ & 3 \\
\hline CWISEP J135937.65-435226.9 $9^{\mathrm{a}}$ & yes & $>18.37$ & $16.13 \pm 0.08$ & $>2.24$ & 70079232 & $18.169 \pm 0.083$ & $15.920 \pm 0.019$ & $2.249 \pm 0.085$ & 1 \\
\hline WISEA J143422.31-083934.2 & yes & $>18.52$ & $16.02 \pm 0.08$ & $>2.50$ & 65978368 & $17.930 \pm 0.068$ & $15.855 \pm 0.019$ & $2.075 \pm 0.071$ & 1 \\
\hline WISEA J151620.39+721745.4 & yes & $18.91 \pm 0.17$ & $16.13 \pm 0.05$ & $2.79 \pm 0.18$ & 65978624 & $18.170 \pm 0.084$ & $15.946 \pm 0.019$ & $2.224 \pm 0.086$ & 1 \\
\hline WISEA J152529.09+605356.5 & yes & $>19.67$ & $15.99 \pm 0.05$ & $>3.68$ & 65978880 & $18.024 \pm 0.073$ & $15.883 \pm 0.019$ & $2.141 \pm 0.076$ & 1 \\
\hline WISEAR J154025.77-113940.8 & yes & $18.20 \pm 0.21$ & $16.06 \pm 0.10$ & $2.14 \pm 0.23$ & 68143360 & $17.417 \pm 0.044$ & $15.927 \pm 0.019$ & $1.489 \pm 0.048$ & 1 \\
\hline WISEA J155349.96+693355.2 & yes & $16.48 \pm 0.03$ & $15.49 \pm 0.03^{\mathrm{b}}$ & $0.99 \pm 0.04$ & 65979136 & $16.324 \pm 0.019$ & $15.458 \pm 0.018$ & $0.865 \pm 0.026$ & 1 \\
\hline WISEA J160516.79+002139.0 & yes & $18.37 \pm 0.20$ & $15.46 \pm 0.05$ & $2.90 \pm 0.20$ & 65979392 & $17.403 \pm 0.043$ & $15.483 \pm 0.018$ & $1.920 \pm 0.047$ & 1 \\
\hline WISEA J161940.51+134752.0 & no & $16.98 \pm 0.06$ & $14.74 \pm 0.03$ & $2.24 \pm 0.06$ & 70019328 & $16.479 \pm 0.021$ & $14.795 \pm 0.016$ & $1.684 \pm 0.026$ & 1 \\
\hline WISEA J162716.41-244355.4 & no & $16.37 \pm 0.06$ & $13.76 \pm 0.02^{\mathrm{b}}$ & $2.61 \pm 0.06$ & 70019584 & $16.052 \pm 0.019$ & $13.951 \pm 0.016$ & $2.102 \pm 0.025$ & 1 \\
\hline WISEA J162852.64+160421.0 & yes & $18.49 \pm 0.21$ & $15.90 \pm 0.06$ & $2.58 \pm 0.22$ & 65980160 & $17.790 \pm 0.060$ & $15.839 \pm 0.019$ & $1.951 \pm 0.063$ & 1 \\
\hline WISEA J163932.75+184049.4 & yes & $18.09 \pm 0.19$ & $15.50 \pm 0.04$ & $2.59 \pm 0.19$ & 65980416 & $17.656 \pm 0.052$ & $15.371 \pm 0.017$ & $2.285 \pm 0.054$ & 1 \\
\hline WISEA J165902.66+274747.9 & no & $>19.23$ & $16.06 \pm 0.07^{\mathrm{b}}$ & $>3.17$ & 70081536 & $17.843 \pm 0.061$ & $15.961 \pm 0.019$ & $1.882 \pm 0.064$ & 1 \\
\hline WISEA J171331.68+245000.9 & yes & $19.12 \pm 0.36$ & $15.98 \pm 0.06$ & $3.14 \pm 0.37$ & 65980672 & $17.524 \pm 0.047$ & $15.842 \pm 0.019$ & $1.681 \pm 0.052$ & 1 \\
\hline WISEA J172907.10-753017.0 & yes & $17.36 \pm 0.07$ & $15.48 \pm 0.04$ & $1.87 \pm 0.08$ & 70080256 & $16.848 \pm 0.027$ & $15.420 \pm 0.018$ & $1.428 \pm 0.032$ & 1 \\
\hline WISEA J175328.55-590447.6 & yes & $>19.00$ & $15.58 \pm 0.05$ & $>3.42$ & 68144640 & $17.336 \pm 0.039$ & $15.186 \pm 0.017$ & $2.150 \pm 0.043$ & 1 \\
\hline WISEA J175701.50+183215.2 & no & $18.49 \pm 0.21$ & $15.72 \pm 0.05^{\mathrm{b}}$ & $2.77 \pm 0.22$ & 68141312 & $17.863 \pm 0.062$ & $15.726 \pm 0.019$ & $2.138 \pm 0.065$ & 1 \\
\hline WISEA J181849.59-470146.9 & yes & $18.51 \pm 0.51$ & $15.14 \pm 0.08^{\mathrm{b}}$ & $3.37 \pm 0.52$ & 65980928 & $17.100 \pm 0.034$ & $15.066 \pm 0.016$ & $2.034 \pm 0.038$ & 3 \\
\hline WISEA J185109.93+671343.7 & no & $17.29 \pm 0.04$ & $16.71 \pm 0.07$ & $0.58 \pm 0.08$ & 65981184 & $17.142 \pm 0.036$ & $16.414 \pm 0.023$ & $0.728 \pm 0.043$ & 1 \\
\hline WISEA J190005.76-310810.9 & yes & $18.42 \pm 0.36$ & $14.92 \pm 0.04$ & $3.50 \pm 0.36$ & 65981440 & $16.852 \pm 0.028$ & $14.787 \pm 0.016$ & $2.064 \pm 0.032$ & 1 \\
\hline WISEA J193054.55-205949.4 & yes & $>18.80$ & $15.24 \pm 0.05^{\mathrm{b}}$ & $>3.56$ & 65981696 & $18.417 \pm 0.104$ & $15.250 \pm 0.017$ & $3.168 \pm 0.105$ & 1 \\
\hline WISEA J201833.67-141720.3 & yes & $>18.57$ & $15.06 \pm 0.04$ & $>3.51$ & 65981952 & $17.166 \pm 0.036$ & $14.997 \pm 0.016$ & $2.168 \pm 0.040$ & 1 \\
\hline WISEA J205921.85+662725.2 & yes & $18.71 \pm 0.18$ & $15.70 \pm 0.04$ & $3.02 \pm 0.18$ & 65982208 & $17.414 \pm 0.042$ & $15.526 \pm 0.018$ & $1.888 \pm 0.046$ & 1 \\
\hline WISEA J211456.86-180519.0 & yes & $18.12 \pm 0.19$ & $14.97 \pm 0.04$ & $3.15 \pm 0.19$ & 65982464 & $16.791 \pm 0.026$ & $14.938 \pm 0.016$ & $1.853 \pm 0.031$ & 1 \\
\hline WISEA J212020.71+303656.2 & no & $17.13 \pm 0.06$ & $15.53 \pm 0.05$ & $1.60 \pm 0.08$ & 70081280 & $16.258 \pm 0.020$ & $15.255 \pm 0.017$ & $1.003 \pm 0.025$ & 1 \\
\hline WISEA J212510.91-730758.8 & yes & $18.27 \pm 0.16$ & $15.62 \pm 0.05$ & $2.65 \pm 0.17$ & 65982720 & $17.372 \pm 0.043$ & $15.561 \pm 0.018$ & $1.812 \pm 0.047$ & 1 \\
\hline WISEA J213810.99+373312.9 & no & $17.32 \pm 0.07$ & $15.75 \pm 0.05$ & $1.57 \pm 0.08$ & 70081024 & $17.281 \pm 0.041$ & $15.888 \pm 0.019$ & $1.394 \pm 0.045$ & 1 \\
\hline WISEA J214025.23-332707.4 & yes & $18.90 \pm 0.51$ & $16.10 \pm 0.10$ & $2.80 \pm 0.52$ & 70080768 & $18.130 \pm 0.082$ & $16.122 \pm 0.021$ & $2.008 \pm 0.086$ & 1 \\
\hline WISEU J215018.46-752053.0 & yes & $18.18 \pm 0.15$ & $15.81 \pm 0.05^{\mathrm{b}}$ & $2.37 \pm 0.16$ & 68140800 & $17.020 \pm 0.036$ & $15.632 \pm 0.018$ & $1.388 \pm 0.041$ & 5 \\
\hline WISEAR J220746.67-503631.7 & yes & $>19.09$ & $16.16 \pm 0.09$ & $>2.94$ & 68141056 & $17.664 \pm 0.052$ & $16.001 \pm 0.019$ & $1.663 \pm 0.056$ & 1 \\
\hline WISEA J221859.33+114644.4 & yes & $17.90 \pm 0.14$ & $15.04 \pm 0.03$ & $2.85 \pm 0.14$ & 65983232 & $16.490 \pm 0.021$ & $14.889 \pm 0.016$ & $1.602 \pm 0.027$ & 1 \\
\hline WISEA J221841.38+143003.4 & yes & $17.23 \pm 0.07$ & $15.15 \pm 0.04$ & $2.07 \pm 0.08$ & 70082304 & $16.896 \pm 0.030$ & $15.281 \pm 0.017$ & $1.615 \pm 0.034$ & 1 \\
\hline WISEA J224319.56-145857.3 & yes & $17.91 \pm 0.24$ & $15.39 \pm 0.05$ & $2.52 \pm 0.24$ & 65984000 & $17.765 \pm 0.058$ & $15.336 \pm 0.018$ & $2.429 \pm 0.060$ & 1 \\
\hline WISEU J224547.11-433341.5 & yes & $\ldots$ & $15.60 \pm 0.05^{\mathrm{b}}$ & $\ldots$ & 68142336 & $\ldots$ & $15.029 \pm 0.017$ & $\ldots$ & 2 \\
\hline
\end{tabular}


Table 2

(Continued)

\begin{tabular}{|c|c|c|c|c|c|c|c|c|c|}
\hline Name & $\begin{array}{l}\text { Motion } \\
\text { Confirmed }\end{array}$ & $\begin{array}{l}\text { W1 } \\
\text { (mag) }\end{array}$ & $\begin{array}{l}\text { W2 } \\
(\mathrm{mag})\end{array}$ & $\begin{array}{l}\mathrm{W} 1-\mathrm{W} 2 \\
(\mathrm{mag})\end{array}$ & AOR & $\begin{array}{l}\text { ch1 } \\
(\mathrm{mag})\end{array}$ & $\begin{array}{l}\text { ch2 } \\
(\mathrm{mag})\end{array}$ & $\begin{array}{c}\mathrm{ch} 1-\mathrm{ch} 2 \\
(\mathrm{mag})\end{array}$ & $\begin{array}{c}\text { WISE } \\
\text { Phot. Reference }^{c}\end{array}$ \\
\hline WISEA J225404.16-265257.5 & yes & $18.20 \pm 0.19$ & $15.38 \pm 0.05$ & $2.81 \pm 0.20$ & 65984512 & $17.467 \pm 0.046$ & $15.200 \pm 0.017$ & $2.267 \pm 0.049$ & 1 \\
\hline WISEA J230930.58+145633.1 & yes & $17.96 \pm 0.14$ & $15.64 \pm 0.06$ & $2.32 \pm 0.15$ & 65984768 & $16.867 \pm 0.027$ & $15.455 \pm 0.018$ & $1.411 \pm 0.033$ & 1 \\
\hline WISEA J233816.47-732929.7 & yes & $18.37 \pm 0.23$ & $15.48 \pm 0.04^{\mathrm{b}}$ & $2.88 \pm 0.23$ & 65985024 & $17.628 \pm 0.052$ & $15.525 \pm 0.018$ & $2.102 \pm 0.055$ & 1 \\
\hline WISEA J235120.62-700025.8 & no & $19.12 \pm 0.38$ & $15.66 \pm 0.04$ & $3.47 \pm 0.38$ & 70083328 & $18.415 \pm 0.109$ & $15.708 \pm 0.019$ & $2.707 \pm 0.111$ & 1 \\
\hline WISEA J235456.63-481440.1 & yes & $18.55 \pm 0.20$ & $15.89 \pm 0.06$ & $2.66 \pm 0.21$ & 65985536 & $18.086 \pm 0.078$ & $15.850 \pm 0.019$ & $2.236 \pm 0.080$ & 1 \\
\hline
\end{tabular}

\section{Notes.}

${ }^{\mathrm{a}}$ The discovery of CWISEP J135937.65-435226.9 was presented in Meisner et al. (2020), but its more recent p14299 Spitzer photometry provided here is deeper than that of Meisner et al. (2020).

${ }^{\mathrm{b}}$ WISE photometry is or may be contaminated by blending.

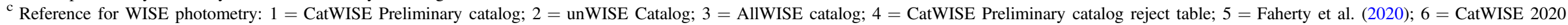
catalog.

(This table is available in machine-readable form.) 


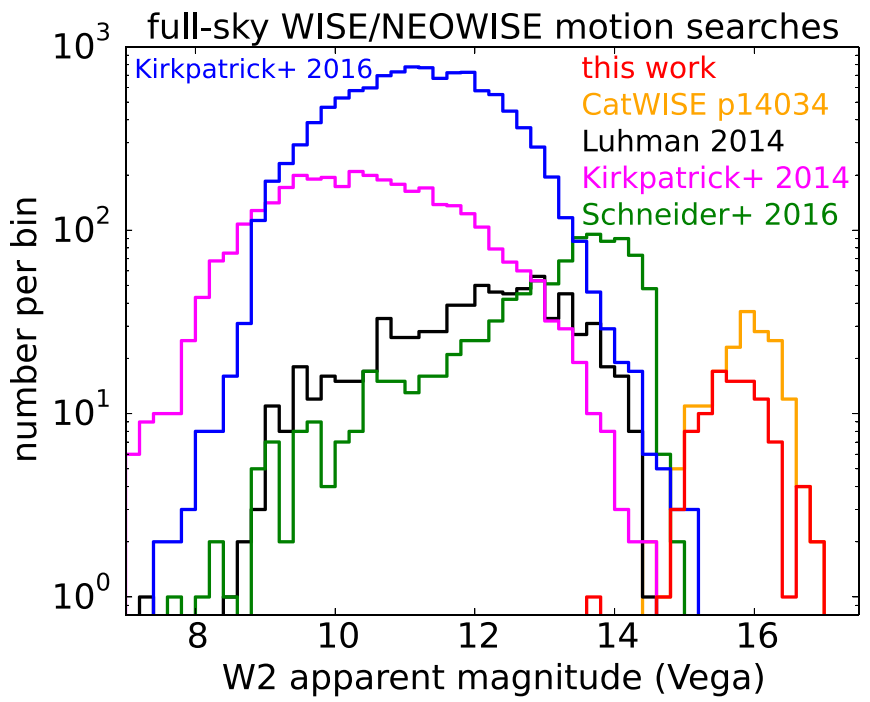

Figure 2. W2 magnitude distributions of full-sky WISE/NEOWISE motion survey samples (logarithmic vertical axis scale). The red histogram represents our 95 Backyard Worlds discoveries followed up with Spitzer p14076 and p14299, plus one with archival Spitzer data (CWISE 0002+6352). The Backyard Worlds targets are much fainter than those of all prior samples except for CatWISE (Spitzer program 14034; Meisner et al. 2020).

searches because they make use of time-resolved unWISE coadd images (Meisner et al. 2018b, 2018c, 2019) spanning the full WISE+NEOWISE baseline.

\section{Spitzer Observing Strategy}

The primary goal of our Spitzer follow-up is to obtain photometric spectral type estimates, which we typically cannot determine from WISE because our targets generally lack secure W1 detections. Spitzer ch1-ch2 color tends to increase monotonically toward later spectral types, and so we can use the Kirkpatrick et al. (2019) relation for spectral type as a function of $\operatorname{ch} 1-\mathrm{ch} 2$ color to phototype our brown dwarf candidates.

We adopted a simplistic observing strategy whereby every target is observed using the exact same Spitzer dithering sequence-no scaling of the total exposure time (or number of dithers) per target was performed based on anticipated Spitzer brightness/color. We opted for a per-target Astronomical Observation Request (AOR) consisting of a "Spiral16" dither pattern with "medium" scale and a $30 \mathrm{~s}$ frame time per channel per dither.

The total exposure time per target in ch1 was engineered to ensure that our Spitzer photometry can distinguish between late $\mathrm{T}$ and $\mathrm{Y}$ dwarfs, even for our faintest targets. The Spitzer color "boundary" between late T and Y dwarfs occurs at ch1-ch2 $\approx 2.4$ mag (Kirkpatrick et al. 2019). In our observation planning, we assume that ch2 $\approx \mathrm{W} 2$, as is typical for mid-T and later brown dwarfs (Kirkpatrick et al. 2019). The faintest $\mathrm{W} 2$ magnitude among our targets is $\mathrm{W} 2=16.78$, so we budget to achieve a ch1 signal-to-noise ratio $(\mathrm{S} / \mathrm{N})$ of 5 near the $\mathrm{T} / \mathrm{Y}$ boundary according to $\operatorname{ch} 1(\mathrm{~S} / \mathrm{N}=5)=16.78+2.4=19.18$. Our past Spitzer observing campaigns (e.g., program 70062) have indicated that obtaining five dithers with $30 \mathrm{~s}$ frame times achieves $\mathrm{S} / \mathrm{N}=5$ at $\mathrm{ch} 1=18.75$. Assuming that depth scales with the square root of the number of dithers, 16 dithers would then go 0.63 mag deeper at $5 \sigma$, yielding an expected $5 \sigma$ ch 1 threshold of $19.38 \mathrm{mag}$, exceeding that desired with a margin of 0.2 mag. This margin can help accommodate depth variations due to factors such as zodiacal background or diffuse emission near the Galactic plane. Our targets have median ch $2 \approx 15.6$ and median $\operatorname{ch} 1-\operatorname{ch} 2 \approx 1.9$ (Table 2 ), so we typically obtain a ch1 uncertainty of $\sim 0.04-0.05$ mag (see Section 7), corresponding to $\operatorname{ch} 1 \mathrm{~S} / \mathrm{N}$ of $\sim 20-25$.

\section{Spitzer Photometry}

Spitzer photometry is performed according to Section 5.1 of Kirkpatrick et al. (2019). In brief, we construct a custom mosaic for each target in each of ch1 and ch2 using the MOsaicker and Point Source EXtractor (MOPEX; Makovoz \& Khan 2005) software package. For a few targets, we specially remove one or a small number of dithers where the ch1 counterpart's immediate vicinity happened to be corrupted by a cosmic ray or glint. We then perform source detection and photometry with MOPEX/APEX (Makovoz \& Marleau 2005). We tabulate both point-response function (PRF) and aperture photometry for extracted sources and apply the appropriate aperture correction to our aperture fluxes to obtain their equivalent total fluxes. Table 2 lists our final Spitzer photometry results, where we have averaged each target's PRF and aperture photometry quantities to arrive at the quoted values. In the case of CWISE $0002+6352$, we simply quote the PRF photometry, since the aperture photometry is contaminated by a nearby source.

We adopted a default source detection threshold of $\mathrm{S} / \mathrm{N}=5$, but two exceptionally red targets required decreasing this threshold to $\mathrm{S} / \mathrm{N}=2$ : WISEU 0503-5648 and WISEA 1930-2059.

WISEU 2245-4333 is severely blended with a brighter neighboring source at our Spitzer epoch, such that we are only able to extract photometry in ch2 but not in ch1. Unfortunately, this means that we cannot obtain a Spitzer-based phototype for this source, or estimates of any other derived quantities that require a ch1-ch2 color measurement (e.g., photometric distance and effective temperature).

\section{Spectroscopic Follow-Up}

\subsection{Magellan FIRE Spectroscopy}

We used the $6.5 \mathrm{~m}$ Baade Magellan telescope and the Folded-port InfraRed Echellette (FIRE; Simcoe et al. 2013) spectrograph to obtain NIR spectra of eight objects on our Spitzer programs (see Table 3). Observations were made for four sources on 2018 December 1 under clear conditions and an additional four sources under poor conditions: one object on 2019 December 10 and three objects on 2019 December 12. For all observations, we used the prism mode and the $0 . " 6$ slit (resolution $\lambda / \Delta \lambda \sim 100$ ) covering the full $0.8-2.5 \mu \mathrm{m}$ band. We observed all objects using a standard ABBA nod pattern with an exposure time of $120 \mathrm{~s}$ per nod. We observed an A0 standard star after each target for telluric corrections and obtained an Ne Ar lamp spectrum for wavelength calibration. At the start of the night we used quartz lamps as dome flats in order to calibrate pixel-to-pixel response. Data were reduced using the FIREHOSE package, which is based on the MASE and Spextool reduction packages (Vacca et al. 2003; Cushing et al. 2004; Bochanski et al. 2009). 


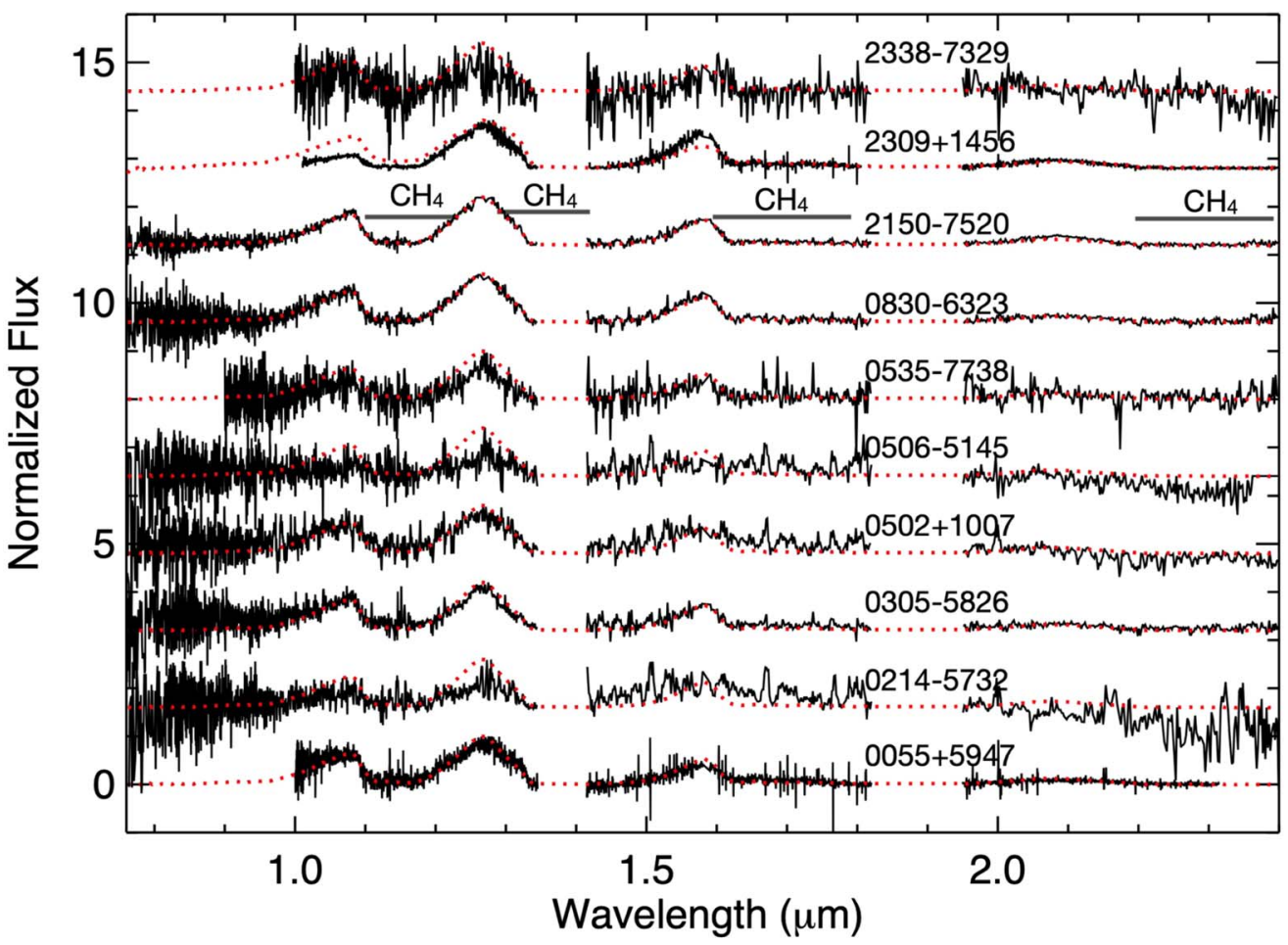

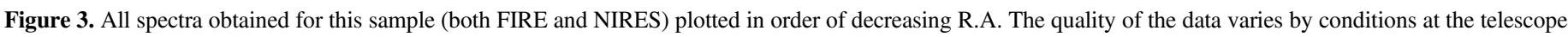

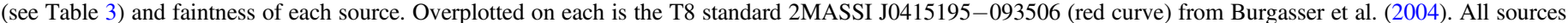
are \pm 1 subtype from a T8; therefore, it is a comparison for all. Regions impacted by telluric absorption are removed (at $\sim 1.4$ and $\sim 1.9 \mu$ m).

Table 3

Details on Spectroscopic Follow-Up

\begin{tabular}{|c|c|c|c|c|c|c|c|c|}
\hline $\begin{array}{l}\text { Name } \\
\text { (1) }\end{array}$ & $\begin{array}{l}\text { Telescope } \\
\text { (2) }\end{array}$ & $\begin{array}{c}\text { Instrument } \\
\text { (3) }\end{array}$ & $\begin{array}{l}\text { Date } \\
(4)\end{array}$ & $\begin{array}{c}\operatorname{Exp}(s) \\
(5)\end{array}$ & $\begin{array}{l}\text { Frames } \\
\text { (6) }\end{array}$ & $\begin{array}{l}\text { Telluric } \\
\text { (7) }\end{array}$ & $\begin{array}{c}\text { Conditions } \\
(8)\end{array}$ & $\begin{array}{l}\text { SpT } \\
(9)\end{array}$ \\
\hline WISEU J005559.88+594745.0 & KECK II & NIRES & 2019 Oct 28 & 300 & 3 & HD 5071 & Clear & $\mathrm{T} 8$ \\
\hline WISEA J021420.18-573245.1 & Baade & FIRE & 2019 Dec 12 & 120 & 6 & HD 23722 & Poor & $>\mathrm{T} 6$ \\
\hline WISEA J050238.28+100750.0 & Baade & FIRE & 2019 Dec 10 & 120 & 6 & HD 287640 & Poor & $>\mathrm{T} 6$ \\
\hline WISEA J050615.56-514521.3 & Baade & FIRE & 2019 Dec 12 & 120 & 5 & HD 60331 & Poor & $\geqslant \mathrm{T} 8$ \\
\hline WISEA J053512.01-773829.7 & Baade & FIRE & 2018 Dec 1 & 120 & 8 & HD 90104 & Clear & $\geqslant \mathrm{T} 8$ \\
\hline WISEA J230930.58+145633.1 & KECK II & NIRES & 2018 Sept 1 & 300 & 12 & HD 7215 & Clear & $\mathrm{T} 8$ \\
\hline WISEA J233816.47-732929.7 & Baade & FIRE & 2019 Dec 12 & 120 & 4 & HD 23722 & Poor & $>\mathrm{T} 6$ \\
\hline
\end{tabular}

Note.

${ }^{a}$ See Faherty et al. (2020) for details on this system.

(This table is available in machine-readable form.)

\subsection{Keck NIRES Spectroscopy}

We used the Keck II telescope and the Near-Infrared Echellette Spectrometer (NIRES; Wilson et al. 2004) on 2019 October 28 and 2018 September 1 to obtain 0.94-2.45 $\mu \mathrm{m}$ NIR spectra of two objects (see Table 3). All components on NIRES are fixed with a 0 ". 55 slit producing resolution $\sim 2700$ data. All sources were identified using the $K$-band finder camera and observed in an ABBA nod pattern. Exposure times and $\mathrm{AB}$ frames acquired are listed in Table 3 . The data were reduced using a modified version of Spextool (Cushing et al. 2004; see also Section 4.4 of Kirkpatrick et al. 2011), following the standard procedure. Wavelength calibration was determined using telluric lines. The spectra of each A-B pair were extracted individually and then combined with the other extracted pairs. The telluric correction procedure was carried out as detailed in Vacca et al. (2003).

\subsection{Spectra Results}

We show all spectra obtained for this Spitzer sample in Figure 3. Objects are arranged in order of decreasing R.A. from top to bottom. Several objects were observed under poor weather conditions (see Table 3), and therefore the quality of their data is diminished, especially at $H$ and $K$ bands (e.g., WISEA J021420.18-573245.1). Regardless, all objects show clear $\mathrm{CH}_{4}$ absorption in $J$ band, and we can confirm all of them to be late $\mathrm{T}$ dwarfs. We compared each object against the $\mathrm{T}$ 
dwarf spectral standards in the SpeX prism library and list our best-fit type in Table 3.

For uniform comparison in Figure 3, we overplot the T8 standard 2MASSI J0415195-093506 (red curve) from Burgasser et al. (2004) on each object. All sources are classified as T8, within one subtype of T8, or broadly defined as a late $\mathrm{T}$ dwarf, and therefore our standard choice serves as a robust comparative object. Several sources are high $\mathrm{S} / \mathrm{N}(>10$ across the wavelength coverage) and well fit to the T8 standard (within one spectral type). These include WISEU J215018.46 -752053.0, which was the subject of Faherty et al. (2020) as it was discovered to be comoving with a Gaia DR2 L1 dwarf; WISEA J083019.97-632305.4, which has Hubble Space Telescope (HST) photometry reported in Bardalez Gagliuffi et al. (2020) consistent with a late T dwarf; and WISEU $\mathrm{J} 005559.88+594745.0$, which is a new comoving companion reported in this paper (see Section 11.6.3). WISEA J083019.97 -632305.4 and WISEU J005559.88+594745.0 both have $J$-, $H$-, and $\mathrm{K}$-band spectra with clear and strong $\mathrm{CH}_{4}$ absorption consistent with the standard, implying that they are field sources with no unusual parameters.

In the case of WISEA J021420.18-573245.1, WISEA J050238.28+100750.0, and WISEA J233816.47-732929.7 the spectra are too noisy to ascertain an exact type. Each of these sources is notably later than T6. In the case of WISEA J050615.56-514521.3, WISEA J053512.01-773829.7, and WISEA J083019.97-632305.4 these sources are all at least T8 but possibly later. Finally, WISEA J230930.58+145633.1 is best fit by the T8 standard. This source and WISE J00559.88 +594745.0 were collected using the NIRES instrument on Keck II. Unlike the FIRE prism spectra shown for all other sources, the Keck NIRES spectra are stitched together from cross-dispersed orders. This can (although not always) lead to poorly fit continuum shapes where order overlaps were deprecated by low-S/N sources. WISEA J230930.58+145633.1 appears to have slightly depleted $J$-band flux and enhanced $H$ - and $K$-band flux; however, this may be due to stitching in the merged spectrum of the source. A bandby-band comparison to the standard is more compatible, and therefore we do not conclude that this object is necessarily peculiar.

\section{Astrometry}

\subsection{Astrometric Analysis Overview}

Because our brown dwarf candidates were selected based on visually perceived source movement, we need a means to quantify our confidence in the motion of each object. This can be accomplished by checking whether or not each target's joint WISE+Spitzer astrometry falls along a consistent linear trajectory. The combination of Spitzer and WISE data points is critical, since our Spitzer follow-up imaging is completely independent of any anomalous occurrence in the WISE data that may have misled our target selection efforts (e.g., noise, artifacts, blending, imperfect blink registration).

Our targets are (by selection) better detected at 4-5 $\mu \mathrm{m}$ than at $3-4 \mu \mathrm{m}$, so we only perform the detailed astrometric analyses of Sections 9.2-9.4 in W2 and ch2. The basic steps of our astrometric analyses are obtaining Gaia-calibrated W2 and ch2 positions spanning the $2010-2019$ time period (Sections 9.2, 9.3) and then fitting these with a linear motion model (Section 9.4). The linear trajectory fitting results can then be used to assess significance of motion as described in Section 9.4. As a by-product, we also obtain more accurate motion measurements than would be possible using WISE data alone.

\subsection{WISE Astrometry}

Full details of our W2 astrometry procedure are provided in Section 8.3 of Meisner et al. (2020). Our WISE astrometry is based on catalogs constructed by running the crowdsource pipeline (Schlafly et al. 2018, 2019) on unWISE co-adds. These unWISE co-adds time-slice the available W2 data in a number of ways, for example, 6-monthly intervals, yearly intervals, the entire pre-hibernation time period, and the entire post-hibernation time period. Detailed definitions of our unWISE time slices for W2 astrometry are provided in Table 5 of Meisner et al. (2020). For each target, we create W2 coadds and catalogs corresponding to all of these different time slices and then choose a subset of the available W2 detections that best covers the entire WISE/NEOWISE time period while never double-counting any W2 observations. The most common choice is simply a pair of W2 astrometric detections: one for the entire pre-hibernation time period co-added together and another for the entire post-hibernation time period coadded together. In the present work we incorporate the sixth year of WISE data (2017 December-2018 December), whereas Meisner et al. (2020) only used 5 yr of WISE data.

The unWISE co-adds simply propagate the native exposurelevel ("Level 1b") world coordinate system (WCS), which can have systematics at the 100-200 mas level. ${ }^{21}$ We therefore recalibrate our W2 astrometry to Gaia DR2 accounting for the Gaia calibrator proper motions. Note that none of our brown dwarf candidates are detected by Gaia; rather, our astrometry is tied to that of brighter/warmer nearby stars present in Gaia. We typically achieve a W2 versus Gaia bright-end scatter of $40-45$ mas per coordinate, just $\sim 1 / 60$ of the WISE pixel side length. The complete set of W2 (R.A., decl.) coordinates used in our WISE+Spitzer linear motion fits is provided in Table 4.

\subsection{Spitzer Astrometry}

Our Spitzer astrometry methodology is as described in Section 8.4 of Meisner et al. (2020); here we provide only a concise summary. Our ch2 astrometry is measured from mosaics rather than single-dither Spitzer frames and proceeds from the same set of extracted catalogs used for our ch2 photometry (Section 7). We do not simply adopt the native WCS of the mosaics, which is inherited from that of the singledither Spitzer frames tied to the Two Micron All Sky Survey (2MASS), since these have astrometry accurate only at the order hundred mas level (Martin et al. 2018). We can achieve much better astrometric accuracy of $\sim 20-25$ mas per coordinate by recalibrating the mosaic WCS to Gaia (Martin et al. 2018; Meisner et al. 2020). In detail, we match the ch2 mosaic extractions to Gaia DR2 using the native WCS and then refit six astrometric parameters per mosaic: the WCS CRPIX coordinates (i.e., translations along the two sky directions) and all four CD matrix elements (i.e., scale factors governing the mosaic pixel size and orientation). ${ }^{22}$ Our fitting minimizes residuals between the WCS-predicted pixel locations of the Gaia DR2 sources (propagated to the relevant Spitzer epoch according to their proper motions) and the measured pixel

\footnotetext{
${ }^{21}$ http://wise2.ipac.caltech.edu/docs/release/allwise/expsup/sec5_2b.html 22 See Greisen \& Calabretta (2002) for precise definitions of these WCS parameters.
} 
Table 4

Recalibrated WISE W2 Positions

\begin{tabular}{|c|c|c|c|c|c|c|}
\hline Name & $\begin{array}{c}\text { R.A. } \\
\text { (deg, ICRS) }\end{array}$ & $\begin{array}{c}\text { Decl. } \\
(\mathrm{deg}, \mathrm{ICRS})\end{array}$ & $\begin{array}{l}\sigma_{\text {R.A. }} \\
\text { (mas) }\end{array}$ & $\begin{array}{l}\sigma_{\text {Decl. }} \\
\text { (mas) }\end{array}$ & $\begin{array}{l}\text { Time } \\
\text { slice }^{\mathrm{a}}\end{array}$ & $\begin{array}{l}\text { Mean } \\
\text { MJD }\end{array}$ \\
\hline \multirow[t]{2}{*}{ CWISE J000229.93+635217.0 } & 0.624757 & 63.871147 & 402 & 389 & post0_2yr & 56983.52 \\
\hline & 0.625715 & 63.871405 & 389 & 374 & post1_2yr & 57716.24 \\
\hline \multirow[t]{6}{*}{ WISEA J001449.96+795116.1 } & 3.708443 & 79.854488 & 432 & 402 & pre & 55359.17 \\
\hline & 3.711609 & 79.854339 & 508 & 476 & post0_1yr & 56813.37 \\
\hline & 3.712429 & 79.854352 & 639 & 590 & post1_1yr & 57172.58 \\
\hline & 3.712981 & 79.854583 & 531 & 489 & post2_1yr & 57526.24 \\
\hline & 3.713487 & 79.854231 & 519 & 483 & post3_1yr & 57900.05 \\
\hline & 3.715139 & 79.854420 & 471 & 433 & post4_1yr & 58245.57 \\
\hline \multirow[t]{2}{*}{ WISEU J001908.31-094323.3 } & 4.785193 & -9.722557 & 658 & 678 & pre & 55454.78 \\
\hline & 4.784726 & -9.723279 & 319 & 319 & post & 57566.42 \\
\hline \multirow[t]{2}{*}{ WISEA J002810.59+521853.1 } & 7.044147 & 52.314851 & 296 & 295 & pre & 55396.93 \\
\hline & 7.044060 & 52.314345 & 287 & 285 & post & 57478.15 \\
\hline \multirow[t]{2}{*}{ WISEA J002909.90+190516.6 } & 7.291288 & 19.088003 & 351 & 357 & pre & 55467.06 \\
\hline & 7.291163 & 19.087455 & 222 & 223 & post & 57484.91 \\
\hline \multirow[t]{2}{*}{ WISEA J003631.29-642735.6 } & 9.130568 & -64.459988 & 370 & 359 & pre & 55438.27 \\
\hline & 9.130869 & -64.460012 & 301 & 285 & post & 57585.75 \\
\hline
\end{tabular}

Note.

${ }^{a}$ See Table 5 of Meisner et al. (2020) for definitions of the WISE/NEOWISE time slices used for W2 astrometry.

(This table is available in its entirety in machine-readable form.)

coordinate centroids. The median per-coordinate astrometric scatter relative to Gaia after our mosaic WCS recalibration is 20 mas.

The complete set of ch2 (R.A., decl.) coordinates used in our WISE+Spitzer linear motion fits is provided in Table 5. The "method number" column of Table 5 is an integer code indicating which set of Gaia DR2 calibrator selection cuts was applied during astrometric recalibration, with these codes defined in Table 6 of Meisner et al. (2020). The $N_{\text {calib }}$ column lists the number of Gaia-Spitzer calibrators employed for each target's mosaic. We sought to always obtain at least five astrometric calibrators per mosaic and only failed to achieve this in one case: WISEA 1627-2443. The lack of calibrators in this field is due to heavy dust extinction within the Gaia bandpass, and in any event WISEA 1627-2443 itself turns out to be an extended piece of nebulosity.

\subsection{Linear Motion Fits}

We combine our W2 and ch2 astrometry by performing linear fits of the motion along both the R.A. and decl. directions. We do not attempt to include a parallactic motion component in these fits. The fits are performed using weighted linear least squares, where the per-coordinate uncertainties are those listed in Tables 4 and 5. The least-squares fitting propagates these positional uncertainties into formal uncertainties on the R.A. and decl. direction linear motions. No outlier rejection is performed, since we generally have only a handful of astrometric data points per target, and all W2 and ch2 detections used for motion fitting were individually vetted in advance. The best-fit linear motions and their uncertainties are provided in Table 6 for all targets detected in our Spitzer ch2 imaging.

A primary aim of our WISE+Spitzer astrometric analysis is to determine quantitatively whether each target is indeed moving. To do this, we adopt the same significance of motion metric and threshold as in Meisner et al. (2020): $\chi_{\text {motion }}^{2}>23.01$, where $\chi_{\text {motion }}^{2}=\mu_{\alpha}^{2} / \sigma_{\mu_{\alpha}}^{2}+\mu_{\delta}^{2} / \sigma_{\mu_{\delta}}^{2}$. This threshold corresponds to a $<10^{-5}$ probability for random scatter in the linear motion measurement to have induced such a large $\chi_{\text {motion }}^{2}$ value for an object that is actually stationary.

Figure 4 shows a histogram of the best-fit total linear motions obtained. Our $\chi_{\text {motion }}^{2}$ threshold tends to be exceeded for $\mu_{\text {tot }} \gtrsim 170$ mas yr $^{-1}$ at the typical W2 magnitude of our sample. A total of 79 of our 97 sample members are motion confirmed according to our $\chi_{\text {motion }}^{2}$ criterion. One, CWISEP 1359-4352, was previously motion confirmed in Meisner et al. (2020). In another two cases, WISEA 1627-2443 and WISEA 1350-8302, we vetoed the motion confirmation after visual inspection of the Spitzer imaging. WISEA 1627-2443 is seen to be spatially extended at Spitzer resolution, and thus our measured positions likely exhibit excess scatter relative to our positional uncertainties that assume pointlike profiles. This source is presumed to be a piece of nebulosity rather than a moving object. Upon more detailed examination of the W2 and ch2 images of WISEA 1350-8302, we determined that the WISE brown dwarf candidate is actually a very faint $\sim 8^{\prime \prime}$ separation pair of Spitzer sources, the redder of which we have listed in Table 5. Therefore, the fitted motion is a spurious artifact of comparing the WISE centroid, which effectively averages the positions of two faint Spitzer sources, against the ch2 position of one of those Spitzer objects.

Since we use the same motion confirmation approach and significance threshold as did the CatWISE team for their p14034 Spitzer follow-up (Meisner et al. 2020), it is interesting to compare our fraction of successful motion confirmations to theirs. A total of 64\% (114/177) of CatWISE p14034 brown dwarf candidates were motion confirmed, versus 79\% (77/97) for our Backyard Worlds Spitzer sample. The higher rate of confirmations for Backyard Worlds likely arises because the CatWISE p14034 sample is fainter by $\sim 0.2-0.3 \mathrm{mag}$ in the median, leading to correspondingly noisier WISE astrometry. 
Table 5

Spitzer ch2 Coordinates Used in Linear Motion Fits

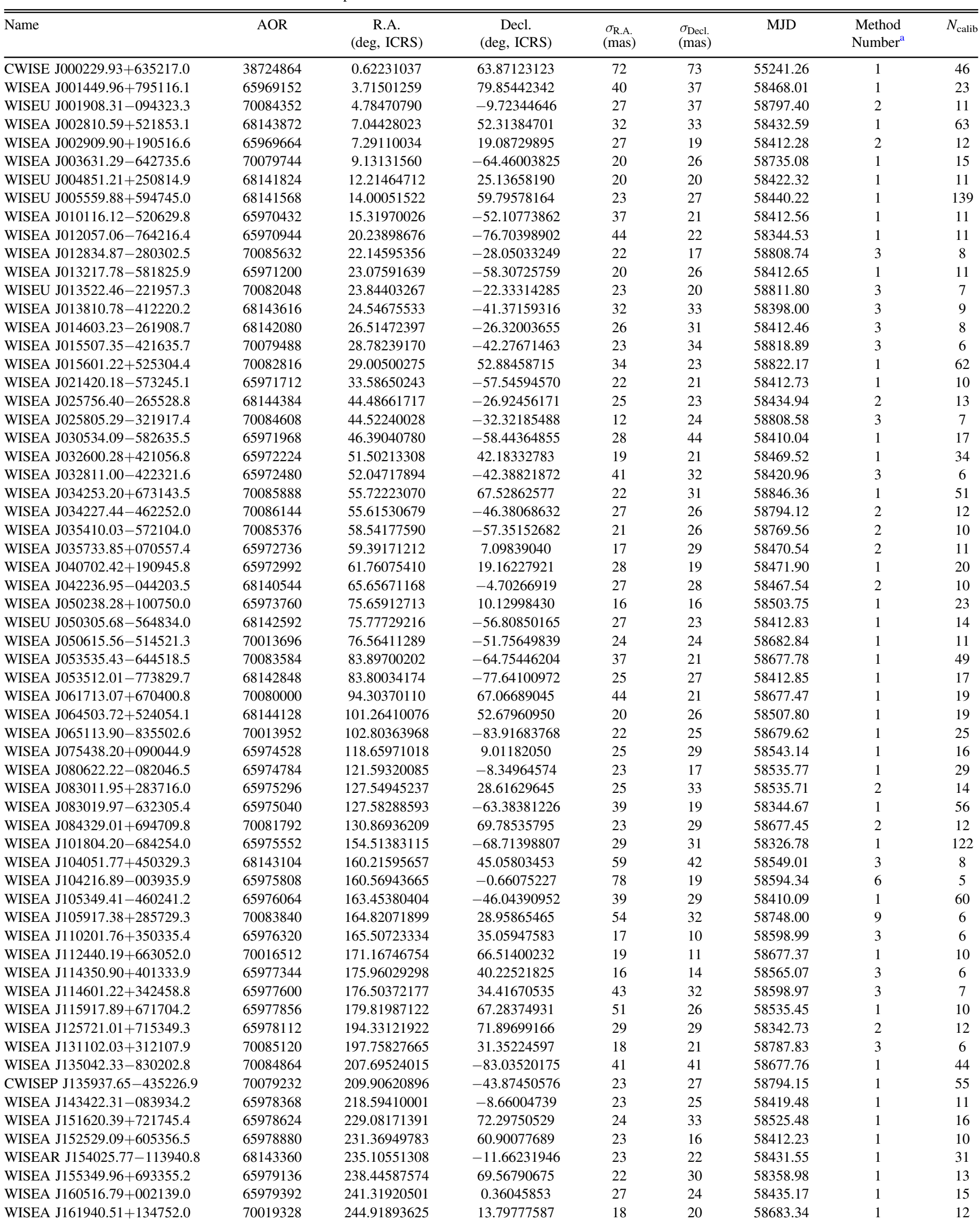


Table 5

(Continued)

\begin{tabular}{|c|c|c|c|c|c|c|c|c|}
\hline Name & AOR & $\begin{array}{c}\text { R.A. } \\
\text { (deg, ICRS) }\end{array}$ & $\begin{array}{c}\text { Decl. } \\
\text { (deg, ICRS) }\end{array}$ & $\begin{array}{l}\sigma_{\text {R.A. }} \\
\text { (mas) }\end{array}$ & $\begin{array}{l}\sigma_{\text {Decl. }} \\
(\mathrm{mas})\end{array}$ & MJD & $\begin{array}{l}\text { Method } \\
\text { Number }^{\mathrm{a}}\end{array}$ & $N_{\text {calit }}$ \\
\hline WISEA J162716.41-244355.4 & 70019584 & 246.81842257 & -24.73230094 & 31 & 14 & 58681.61 & 9 & 4 \\
\hline WISEA J162852.64+160421.0 & 65980160 & 247.21956184 & 16.07014592 & 17 & 27 & 58323.79 & 1 & 14 \\
\hline WISEA J165902.66+274747.9 & 70081536 & 254.76088341 & 27.79695337 & 33 & 32 & 58677.16 & 1 & 11 \\
\hline WISEA J171331.68+245000.9 & 65980672 & 258.38136764 & 24.83425966 & 20 & 29 & 58324.56 & 1 & 23 \\
\hline WISEA J172907.10-753017.0 & 70080256 & 262.28008923 & -75.50523721 & 39 & 20 & 58677.55 & 1 & 54 \\
\hline WISEA J181849.59-470146.9 & 65980928 & 274.70680026 & -47.03089410 & 23 & 21 & 58471.57 & 1 & 117 \\
\hline WISEA J185109.93+671343.7 & 65981184 & 282.79034267 & 67.22875209 & 62 & 30 & 58325.08 & 1 & 30 \\
\hline WISEA J190005.76-310810.9 & 65981440 & 285.02396090 & -31.13711842 & 22 & 16 & 58484.17 & 1 & 137 \\
\hline WISEA J193054.55-205949.4 & 65981696 & 292.72484856 & -20.99948094 & 21 & 30 & 58356.43 & 1 & 67 \\
\hline WISEA J201833.67-141720.3 & 65981952 & 304.64040610 & -14.28865037 & 20 & 19 & 58508.47 & 1 & 43 \\
\hline WISEA J205921.85+662725.2 & 65982208 & 314.83817793 & 66.45691651 & 33 & 41 & 58404.81 & 1 & 43 \\
\hline WISEA J214025.23-332707.4 & 70080768 & 325.10661695 & -33.45339899 & 23 & 25 & 58735.46 & 1 & 16 \\
\hline WISEU J215018.46-752053.0 & 68140800 & 327.58089437 & -75.34866889 & 40 & 17 & 58459.16 & 1 & 15 \\
\hline WISEAR J220746.67-503631.7 & 68141056 & 331.94492853 & -50.61225168 & 26 & 26 & 58509.50 & 2 & 14 \\
\hline WISEA J221859.33+114644.4 & 65983232 & 334.74775599 & 11.77832823 & 11 & 19 & 58407.08 & 1 & 14 \\
\hline WISEA J221841.38+143003.4 & 70082304 & 334.67136398 & 14.50036772 & 13 & 29 & 58766.28 & 1 & 17 \\
\hline WISEA J224319.56-145857.3 & 65984000 & 340.83216229 & -14.98390871 & 30 & 20 & 58411.46 & 2 & 12 \\
\hline WISEU J224547.11-433341.5 & 68142336 & 341.44819598 & -43.56205682 & 27 & 26 & 58523.84 & 1 & 11 \\
\hline WISEA J225404.16-265257.5 & 65984512 & 343.51885430 & -26.88270278 & 23 & 44 & 58410.00 & 3 & 10 \\
\hline WISEA J230930.58+145633.1 & 65984768 & 347.37899411 & 14.94148799 & 41 & 34 & 58412.32 & 2 & 11 \\
\hline WISEA J233816.47-732929.7 & 65985024 & 354.57437439 & -73.49193575 & 19 & 21 & 58412.39 & 1 & 11 \\
\hline WISEA J235120.62-700025.8 & 70083328 & 357.83542855 & -70.00743644 & 25 & 43 & 58711.33 & 1 & 10 \\
\hline WISEA J235456.63-481440.1 & 65985536 & 358.73728759 & -48.24501823 & 40 & 40 & 58412.36 & 1 & 11 \\
\hline
\end{tabular}

Notes. $N_{\text {calib }}$ is the number of Gaia DR2 sources used for Spitzer ch2 astrometric recalibration. See Section 8.4.1 of Meisner et al. (2020) for additional details.

a The astrometric calibrator selection "method number" here refers to the method number listed in Table 6 of Meisner et al. (2020).

(This table is available in machine-readable form.)

\section{Near-infrared Photometry}

\subsection{Mont-Mégantic/CPAPIR Follow-up}

We observed 42 of our brown dwarf candidates in the MKO $J$ band with the Caméra PAnoramique Proche Infra-Rouge (CPAPIR; Artigau et al. 2004) wide-field $\left(30^{\prime} \times 30^{\prime}\right)$ NIR camera located at the Observatoire du Mont-Mégantic (OMM) $1.6 \mathrm{~m}$ observatory (Racine 1978) between 2018 May and 2020 January. Ten of these OMM targets were well detected $(>4 \sigma)$, 11 were marginally detected $(<4 \sigma)$, and 21 were not detected.

Targets were always observed near the center of the lower right quadrant of the CPAPIR camera because the amplifier of the upper right quadrant is malfunctioning. The data were reduced with a custom Python pipeline that was standardized for CPAPIR/OMM data. Images are flat-fielded with a standard white field inside the telescope dome observed at the start or end of the night. A sky image is built from a median of the observations, taken across small, random dithering patterns and where all sources at the 2MASS positions are masked. The sky image is subtracted from each individual frame, and a linear astrometric solution is obtained with a local implementation of the http://astrometry.net tool (Lang et al. 2010) anchored on index files built from Gaia DR2 data (Gaia Collaboration et al. 2018; Lindegren et al. 2018). Individual images are then median combined into a final frame, and a new astrometric solution is built from http://astrometry.net and Gaia DR2 including second-degree distortions as Simple Imaging Polynomial polynomials.

Aperture photometry was calculated for each target with a custom IDL pipeline using an aperture twice as large as the FWHM reported by the OMM data reduction pipeline (1" 7-4!"9 with a median of 2!" 1$)$, centered at the position predicted from the WISE+Spitzer motion solutions. Using twice the FWHM ensures that small deviations in predicted versus observed positions do not affect the photometric measurement, without needing to estimate a new centroid for very faint targets. The background sky was estimated from the median of an annulus with inner and outer radii 3 and 6 times as large as the photometric aperture, respectively.

Photometric zero-points were estimated by measuring the flux of all 2MASS catalog entries within the field of view (while avoiding the outermost 250 pixel edges) and calculating the median zero-point that translates from CPAPIR $J$-band fluxes to 2MASS $J$-band magnitudes. Limiting $5 \sigma$ depths were calculated for nondetections by sampling 1000 uniformrandom positions in the field of view while avoiding 2MASS entries. The average of the absolute flux values at 84th and 16th percentiles of the random distribution multiplied by 5 was then transformed to a $5 \sigma$ detection limit in magnitudes with the 
Linear Motion Fit Results Combining WISE and Spitzer Astrometry

\begin{tabular}{|c|c|c|c|c|c|c|c|c|c|c|c|c|}
\hline Name & $\begin{array}{c}\alpha_{0} \\
\text { (deg, ICRS) }\end{array}$ & $\begin{array}{c}\delta_{0} \\
\text { (deg, ICRS) }\end{array}$ & $\begin{array}{c}\sigma_{\alpha_{0}} \\
\text { (mas) }\end{array}$ & $\begin{array}{c}\sigma_{\delta_{0}} \\
(\mathrm{mas})\end{array}$ & $\mathrm{MJD}_{0}$ & $\begin{array}{c}\mu_{\alpha} \\
\left(\operatorname{mas~yr}^{-1}\right)\end{array}$ & $\begin{array}{c}\mu_{\delta} \\
\left(\operatorname{mas~yr}^{-1}\right)\end{array}$ & $\begin{array}{c}\mu_{\text {tot }} \\
\left(\operatorname{mas~yr}^{-1}\right)\end{array}$ & $\chi_{\text {motion }}^{2}$ & $\chi^{2}$ & dof & $\chi_{\nu}^{2}$ \\
\hline CWISE J000229.93+635217.0 & 0.6225026 & 63.8712344 & 70 & 71 & 55379.85 & $802 \pm 49$ & $44 \pm 47$ & $803 \pm 49$ & 268.7 & 2.53 & 2 & 1.26 \\
\hline WISEA J001449.96+795116.1 & 3.7149072 & 79.8544230 & 40 & 37 & 58416.60 & $487 \pm 44$ & $-9 \pm 41$ & $487 \pm 44$ & 123.1 & 5.15 & 10 & 0.52 \\
\hline WISEU J001908.31-094323.3 & 4.7847093 & -9.7234432 & 28 & 37 & 58778.24 & $-126 \pm 58$ & $-286 \pm 59$ & $312 \pm 59$ & 28.5 & 4.04 & 2 & 2.02 \\
\hline WISEA J002810.59+521853.1 & 7.0442758 & 52.3138656 & 33 & 33 & 58383.44 & $49 \pm 34$ & $-458 \pm 34$ & $461 \pm 34$ & 181.9 & 6.43 & 2 & 3.22 \\
\hline WISEA J002909.90+190516.6 & 7.2911017 & 19.0873034 & 27 & 19 & 58391.51 & $-80 \pm 39$ & $-296 \pm 40$ & $306 \pm 40$ & 59.4 & 0.90 & 2 & 0.45 \\
\hline WISEA J003631.29-642735.6 & 9.1313102 & -64.4600379 & 20 & 26 & 58715.36 & $143 \pm 38$ & $-22 \pm 37$ & $144 \pm 38$ & 14.6 & 0.78 & 2 & 0.39 \\
\hline WISEU J004851.21+250814.9 & 12.2145993 & 25.1366132 & 20 & 20 & 58367.15 & $1009 \pm 21$ & $-728 \pm 21$ & $1244 \pm 21$ & 3446.9 & 8.49 & 10 & 0.85 \\
\hline WISEU J005559.88+594745.0 & 14.0004779 & 59.7957828 & 23 & 27 & 58375.91 & $408 \pm 25$ & $-24 \pm 25$ & $409 \pm 25$ & 275.5 & 12.59 & 10 & 1.26 \\
\hline WISEA J010116.12-520629.8 & 15.3196862 & -52.1077423 & 37 & 21 & 58392.14 & $673 \pm 45$ & $236 \pm 44$ & $713 \pm 45$ & 253.9 & 7.94 & 10 & 0.79 \\
\hline WISEA J012057.06-764216.4 & 20.2389720 & -76.7039955 & 43 & 22 & 58313.86 & $139 \pm 43$ & $271 \pm 40$ & $305 \pm 41$ & 56.7 & 0.17 & 2 & 0.08 \\
\hline WISEA J012834.87-280302.5 & 22.1459505 & -28.0503342 & 22 & 18 & 58793.11 & $211 \pm 37$ & $138 \pm 37$ & $252 \pm 37$ & 46.2 & 0.52 & 2 & 0.26 \\
\hline WISEA J013217.78-581825.9 & 23.0759047 & -58.3072568 & 21 & 26 & 58393.05 & $438 \pm 43$ & $-43 \pm 41$ & $440 \pm 43$ & 106.4 & 6.11 & 10 & 0.61 \\
\hline WISEU J013522.46-221957.3 & 23.8440303 & -22.3331396 & 24 & 20 & 58798.63 & $199 \pm 42$ & $-317 \pm 43$ & $374 \pm 43$ & 77.2 & 0.93 & 2 & 0.47 \\
\hline WISEA J013810.78-412220.2 & 24.5467497 & -41.3715961 & 33 & 33 & 58386.43 & $554 \pm 82$ & $348 \pm 83$ & $655 \pm 82$ & 63.3 & 2.24 & 2 & 1.12 \\
\hline WISEA J014603.23-261908.7 & 26.5147183 & -26.3200316 & 26 & 32 & 58397.66 & $465 \pm 58$ & $-418 \pm 59$ & $625 \pm 58$ & 114.9 & 0.49 & 2 & 0.25 \\
\hline WISEA J015507.35-421635.7 & 28.7823850 & -42.2767135 & 23 & 35 & 58805.52 & $486 \pm 55$ & $-88 \pm 55$ & $494 \pm 55$ & 81.4 & 0.21 & 2 & 0.11 \\
\hline WISEA J015601.22+525304.4 & 29.0049998 & 52.8845881 & 34 & 23 & 58769.24 & $2 \pm 27$ & $-12 \pm 27$ & $12 \pm 27$ & 0.2 & 5.08 & 2 & 2.54 \\
\hline WISEA J021420.18-573245.1 & 33.5864956 & -57.5459455 & 23 & 21 & 58403.07 & $529 \pm 55$ & $-36 \pm 53$ & $530 \pm 55$ & 93.7 & 0.75 & 2 & 0.37 \\
\hline WISEA J025756.40-265528.8 & 44.4866123 & -26.9245623 & 26 & 24 & 58424.03 & $541 \pm 57$ & $93 \pm 58$ & $549 \pm 57$ & 93.5 & 0.54 & 2 & 0.27 \\
\hline WISEA J025805.29-321917.4 & 44.5223944 & -32.3218519 & 12 & 24 & 58775.48 & $183 \pm 19$ & $-115 \pm 19$ & $216 \pm 19$ & 127.4 & 5.51 & 2 & 2.76 \\
\hline WISEA J030534.09-582635.5 & 46.3904512 & -58.4436436 & 28 & 42 & 58329.36 & $-363 \pm 30$ & $-185 \pm 29$ & $407 \pm 30$ & 185.0 & 10.79 & 12 & 0.90 \\
\hline WISEA J032600.28+421056.8 & 51.5021296 & 42.1833222 & 20 & 21 & 58451.49 & $228 \pm 38$ & $410 \pm 39$ & $469 \pm 39$ & 147.3 & 3.34 & 2 & 1.67 \\
\hline WISEA J032811.00-422321.6 & 52.0471675 & -42.3882280 & 41 & 32 & 58392.89 & $407 \pm 50$ & $435 \pm 50$ & $595 \pm 50$ & 140.3 & 0.07 & 2 & 0.03 \\
\hline WISEA J034253.20+673143.5 & 55.7222242 & 67.5286251 & 23 & 31 & 58803.22 & $80 \pm 29$ & $-18 \pm 30$ & $82 \pm 29$ & 8.1 & 3.05 & 2 & 1.52 \\
\hline WISEA J034227.44-462252.0 & 55.6153003 & -46.3806890 & 28 & 27 & 58769.74 & $250 \pm 40$ & $154 \pm 41$ & $294 \pm 40$ & 52.9 & 0.50 & 2 & 0.25 \\
\hline WISEA J035410.03-572104.0 & 58.5417751 & -57.3515241 & 22 & 26 & 58750.33 & $10 \pm 43$ & $-170 \pm 42$ & $171 \pm 42$ & 16.7 & 1.97 & 2 & 0.98 \\
\hline WISEA J035733.85+070557.4 & 59.3917084 & 7.0983951 & 18 & 30 & 58452.48 & $275 \pm 39$ & $-361 \pm 41$ & $454 \pm 41$ & 125.5 & 4.45 & 10 & 0.44 \\
\hline WISEA J040702.42+190945.8 & 61.7607504 & 19.1622810 & 28 & 20 & 58458.11 & $289 \pm 49$ & $-178 \pm 51$ & $340 \pm 49$ & 47.8 & 0.95 & 2 & 0.48 \\
\hline WISEA J042236.95-044203.5 & 65.6566997 & -4.7026620 & 27 & 29 & 58454.14 & $1150 \pm 53$ & $-695 \pm 55$ & $1344 \pm 54$ & 625.6 & 2.58 & 4 & 0.64 \\
\hline WISEA J050238.28+100750.0 & 75.6591290 & 10.1299861 & 16 & 17 & 58492.12 & $-177 \pm 36$ & $-223 \pm 38$ & $284 \pm 38$ & 57.1 & 1.92 & 2 & 0.96 \\
\hline WISEU J050305.68-564834.0 & 75.7772405 & -56.8085133 & 27 & 24 & 58367.04 & $801 \pm 28$ & $322 \pm 28$ & $864 \pm 28$ & 953.4 & 16.91 & 10 & 1.69 \\
\hline WISEA J050615.56-514521.3 & 76.5641167 & -51.7564942 & 24 & 24 & 58659.17 & $-127 \pm 36$ & $-239 \pm 37$ & $271 \pm 37$ & 53.4 & 0.12 & 2 & 0.06 \\
\hline WISEA J053535.43-644518.5 & 83.8970611 & -64.7545225 & 34 & 20 & 58370.15 & $-116 \pm 11$ & $260 \pm 10$ & $284 \pm 10$ & 773.0 & 0.85 & 2 & 0.42 \\
\hline WISEA J053512.01-773829.7 & 83.8003381 & -77.6410178 & 26 & 27 & 58374.70 & $13 \pm 30$ & $251 \pm 32$ & $251 \pm 32$ & 59.9 & 3.15 & 2 & 1.58 \\
\hline WISEA J061713.07+670400.8 & 94.3037035 & 67.0668897 & 44 & 22 & 58655.33 & $-113 \pm 40$ & $24 \pm 43$ & $116 \pm 40$ & 8.2 & 4.56 & 2 & 2.28 \\
\hline WISEA J064503.72+524054.1 & 101.2641238 & 52.6796522 & 20 & 26 & 58450.69 & $-314 \pm 21$ & $-945 \pm 23$ & $996 \pm 23$ & 1887.8 & 9.48 & 10 & 0.95 \\
\hline WISEA J065113.90-835502.6 & 102.8036976 & -83.9168458 & 23 & 25 & 58631.78 & $-175 \pm 26$ & $230 \pm 27$ & $290 \pm 27$ & 118.4 & 0.47 & 2 & 0.24 \\
\hline WISEA J075438.20+090044.9 & 118.6597088 & 9.0118237 & 25 & 29 & 58528.49 & $166 \pm 52$ & $-280 \pm 55$ & $326 \pm 54$ & 36.4 & 2.18 & 2 & 1.09 \\
\hline WISEA J080622.22-082046.5 & 121.5931954 & -8.3496258 & 24 & 18 & 58516.33 & $296 \pm 34$ & $-1366 \pm 36$ & $1398 \pm 36$ & 1512.3 & 9.87 & 10 & 0.99 \\
\hline WISEA J083011.95+283716.0 & 127.5494547 & 28.6163163 & 25 & 33 & 58523.11 & $-189 \pm 58$ & $-2053 \pm 62$ & $2062 \pm 62$ & 1120.6 & 4.38 & 10 & 0.44 \\
\hline WISEA J083019.97-632305.4 & 127.5828973 & -63.3838313 & 39 & 19 & 58297.09 & $-83 \pm 28$ & $501 \pm 28$ & $508 \pm 28$ & 334.0 & 10.09 & 10 & 1.01 \\
\hline WISEA J084329.01+694709.8 & 130.8693699 & 69.7853611 & 23 & 29 & 58662.96 & $-234 \pm 50$ & $-278 \pm 53$ & $363 \pm 52$ & 49.0 & 0.25 & 2 & 0.12 \\
\hline
\end{tabular}


(Continued)

\begin{tabular}{|c|c|c|c|c|c|c|c|c|c|c|c|c|}
\hline Name & $\begin{array}{c}\alpha_{0} \\
\text { (deg, ICRS) }\end{array}$ & $\begin{array}{c}\delta_{0} \\
(\mathrm{deg}, \text { ICRS })\end{array}$ & $\begin{array}{c}\sigma_{\alpha_{0}} \\
\text { (mas) }\end{array}$ & $\begin{array}{c}\sigma_{\delta_{0}} \\
\text { (mas) }\end{array}$ & $\mathrm{MJD}_{0}$ & $\begin{array}{c}\mu_{\alpha} \\
\left(\operatorname{mas~yr}^{-1}\right)\end{array}$ & $\begin{array}{c}\mu_{\delta} \\
\left(\operatorname{mas~yr}^{-1}\right)\end{array}$ & $\begin{array}{c}\mu_{\text {tot }} \\
\left(\mathrm{mas} \mathrm{yr}^{-1}\right)\end{array}$ & $\chi_{\text {motion }}^{2}$ & $\chi^{2}$ & dof & $\chi_{\nu}^{2}$ \\
\hline WISEA J101804.20-684254.0 & 154.5139496 & -68.7140252 & 28 & 30 & 58239.38 & $-608 \pm 26$ & $536 \pm 26$ & $810 \pm 26$ & 1002.9 & 10.97 & 10 & 1.10 \\
\hline WISEA J104051.77+450329.3 & 160.2159462 & 45.0580445 & 56 & 42 & 58406.68 & $62 \pm 31$ & $-73 \pm 31$ & $96 \pm 31$ & 9.5 & 1.57 & 2 & 0.79 \\
\hline WISEA J104216.89-003935.9 & 160.5694386 & -0.6607485 & 77 & 19 & 58579.15 & $-361 \pm 51$ & $-342 \pm 53$ & $497 \pm 52$ & 92.2 & 1.08 & 2 & 0.54 \\
\hline WISEA J105349.41-460241.2 & 163.4538550 & -46.0439285 & 38 & 29 & 58340.00 & $-664 \pm 31$ & $370 \pm 32$ & $761 \pm 31$ & 606.4 & 2.54 & 6 & 0.42 \\
\hline WISEA J105917.38+285729.3 & 164.8207434 & 28.9586486 & 53 & 33 & 58703.56 & $-592 \pm 46$ & $191 \pm 47$ & $622 \pm 46$ & 185.1 & 1.09 & 2 & 0.55 \\
\hline WISEA J110201.76+350335.4 & 165.5072344 & 35.0594772 & 17 & 11 & 58587.00 & $-66 \pm 29$ & $-159 \pm 30$ & $172 \pm 30$ & 33.8 & 5.89 & 10 & 0.59 \\
\hline WISEA J112440.19+663052.0 & 171.1674677 & 66.5140041 & 19 & 12 & 58662.24 & $10 \pm 27$ & $-152 \pm 28$ & $152 \pm 28$ & 29.0 & 0.34 & 2 & 0.17 \\
\hline WISEA J114350.90+401333.9 & 175.9602973 & 40.2252208 & 16 & 15 & 58556.32 & $-492 \pm 41$ & $-384 \pm 43$ & $624 \pm 41$ & 228.5 & 10.12 & 10 & 1.01 \\
\hline WISEA J114601.22+342458.8 & 176.5037433 & 34.4166991 & 43 & 32 & 58547.09 & $-470 \pm 39$ & $128 \pm 41$ & $487 \pm 39$ & 156.3 & 9.26 & 10 & 0.93 \\
\hline WISEA J115917.89+671704.2 & 179.8199024 & 67.2837552 & 50 & 27 & 58513.81 & $-774 \pm 59$ & $-338 \pm 60$ & $845 \pm 60$ & 201.3 & 5.01 & 10 & 0.50 \\
\hline WISEA J125721.01+715349.3 & 194.3312702 & 71.8969922 & 29 & 29 & 58322.64 & $-952 \pm 54$ & $-25 \pm 53$ & $952 \pm 54$ & 310.3 & 5.17 & 2 & 2.58 \\
\hline WISEA J131102.03+312107.9 & 197.7582770 & 31.3522460 & 18 & 22 & 58773.53 & $-42 \pm 38$ & $10 \pm 40$ & $44 \pm 38$ & 1.3 & 1.31 & 2 & 0.66 \\
\hline WISEA J135042.33-830202.8 & 207.6951222 & -83.0351927 & 42 & 41 & 58658.13 & $909 \pm 63$ & $-533 \pm 62$ & $1054 \pm 62$ & 284.4 & 10.52 & 2 & 5.26 \\
\hline CWISEP J135937.65-435226.9 & 209.9062116 & -43.8745052 & 23 & 27 & 58784.91 & $-303 \pm 65$ & $-141 \pm 68$ & $334 \pm 65$ & 26.0 & 3.91 & 2 & 1.96 \\
\hline WISEA J143422.31-083934.2 & 218.5940972 & -8.6600460 & 23 & 25 & 58412.23 & $503 \pm 69$ & $-236 \pm 73$ & $555 \pm 70$ & 63.2 & 0.24 & 2 & 0.12 \\
\hline WISEA J151620.39+721745.4 & 229.0817502 & 72.2974880 & 24 & 33 & 58490.15 & $-429 \pm 37$ & $654 \pm 35$ & $782 \pm 36$ & 474.8 & 3.65 & 10 & 0.36 \\
\hline WISEA J152529.09+605356.5 & 231.3695088 & 60.9007668 & 23 & 17 & 58395.04 & $-365 \pm 38$ & $770 \pm 37$ & $852 \pm 37$ & 516.8 & 4.70 & 10 & 0.47 \\
\hline WISEAR J154025.77-113940.8 & 235.1055164 & -11.6623176 & 23 & 23 & 58426.07 & $-777 \pm 74$ & $-409 \pm 77$ & $878 \pm 75$ & 138.1 & 0.59 & 2 & 0.30 \\
\hline WISEA J155349.96+693355.2 & 238.4459734 & 69.5678794 & 22 & 30 & 58332.37 & $-1684 \pm 56$ & $1348 \pm 53$ & $2157 \pm 55$ & 1549.3 & 5.05 & 8 & 0.63 \\
\hline WISEA J160516.79+002139.0 & 241.3192107 & 0.3604616 & 27 & 24 & 58409.22 & $-295 \pm 38$ & $-157 \pm 40$ & $334 \pm 38$ & 76.4 & 0.04 & 2 & 0.02 \\
\hline WISEA J161940.51+134752.0 & 244.9189341 & 13.7977748 & 18 & 20 & 58614.57 & $45 \pm 16$ & $8 \pm 17$ & $45 \pm 16$ & 7.7 & 2.17 & 2 & 1.08 \\
\hline WISEA J162716.41-244355.4 & 246.8184113 & -24.7322913 & 29 & 14 & 58575.18 & $39 \pm 15$ & $-105 \pm 14$ & $112 \pm 14$ & 65.1 & 25.16 & 10 & 2.52 \\
\hline WISEA J162852.64+160421.0 & 247.2195602 & 16.0701545 & 18 & 28 & 58313.18 & $154 \pm 49$ & $-1047 \pm 53$ & $1059 \pm 53$ & 401.8 & 11.69 & 10 & 1.17 \\
\hline WISEA J163932.75+184049.4 & 249.8851790 & 18.6803400 & 21 & 21 & 58303.49 & $-523 \pm 35$ & $28 \pm 37$ & $524 \pm 35$ & 222.1 & 4.25 & 10 & 0.42 \\
\hline WISEA J165902.66+274747.9 & 254.7608835 & 27.7969502 & 33 & 32 & 58653.69 & $-32 \pm 53$ & $149 \pm 56$ & $152 \pm 56$ & 7.4 & 2.09 & 2 & 1.04 \\
\hline WISEA J171331.68+245000.9 & 258.3813705 & 24.8342589 & 21 & 29 & 58314.64 & $-304 \pm 61$ & $214 \pm 65$ & $372 \pm 63$ & 35.2 & 4.14 & 2 & 2.07 \\
\hline WISEA J172907.10-753017.0 & 262.2800827 & -75.5052306 & 39 & 21 & 58628.40 & $46 \pm 27$ & $-181 \pm 28$ & $187 \pm 28$ & 44.0 & 0.26 & 2 & 0.13 \\
\hline WISEA J175328.55-590447.6 & 268.3680988 & -59.0805770 & 24 & 21 & 58445.32 & $-197 \pm 35$ & $-328 \pm 36$ & $382 \pm 36$ & 112.8 & 4.02 & 10 & 0.40 \\
\hline WISEA J175701.50+183215.2 & 269.2565871 & 18.5374332 & 25 & 19 & 58443.86 & $114 \pm 44$ & $-81 \pm 47$ & $140 \pm 45$ & 9.6 & 0.58 & 2 & 0.29 \\
\hline WISEA J181849.59-470146.9 & 274.7067977 & -47.0308803 & 23 & 22 & 58433.15 & $29 \pm 28$ & $-517 \pm 29$ & $518 \pm 29$ & 311.5 & 10.01 & 2 & 5.01 \\
\hline WISEA J185109.93+671343.7 & 282.7903397 & 67.2287532 & 61 & 30 & 58301.83 & $52 \pm 74$ & $-97 \pm 70$ & $110 \pm 71$ & 2.4 & 1.55 & 2 & 0.77 \\
\hline WISEA J190005.76-310810.9 & 285.0239614 & -31.1371133 & 22 & 16 & 58460.85 & $-26 \pm 28$ & $-301 \pm 30$ & $303 \pm 30$ & 101.0 & 0.82 & 2 & 0.41 \\
\hline WISEA J193054.55-205949.4 & 292.7248684 & -20.9994605 & 21 & 30 & 58331.41 & $-994 \pm 36$ & $-1098 \pm 38$ & $1481 \pm 38$ & 1561.2 & 4.71 & 10 & 0.47 \\
\hline WISEA J201833.67-141720.3 & 304.6404052 & -14.2886541 & 21 & 20 & 58482.80 & $48 \pm 32$ & $191 \pm 33$ & $197 \pm 33$ & 35.4 & 0.07 & 2 & 0.04 \\
\hline WISEA J205921.85+662725.2 & 314.8382734 & 66.4569167 & 32 & 40 & 58303.22 & $-511 \pm 28$ & $-31 \pm 27$ & $512 \pm 28$ & 326.5 & 3.95 & 2 & 1.98 \\
\hline WISEA J211456.86-180519.0 & 318.7357753 & -18.0897946 & 13 & 14 & 58518.15 & $-460 \pm 35$ & $-473 \pm 36$ & $660 \pm 35$ & 349.8 & 2.77 & 10 & 0.28 \\
\hline WISEA J212020.71+303656.2 & 320.0864070 & 30.6156283 & 16 & 21 & 58747.66 & $6 \pm 33$ & $10 \pm 33$ & $12 \pm 33$ & 0.1 & 0.01 & 2 & 0.00 \\
\hline WISEA J212510.91-730758.8 & 321.2981397 & -73.1339610 & 31 & 31 & 58306.39 & $325 \pm 41$ & $-464 \pm 41$ & $567 \pm 41$ & 189.7 & 1.60 & 2 & 0.80 \\
\hline WISEA J213810.99+373312.9 & 324.5458272 & 37.5537319 & 24 & 28 & 58731.84 & $-21 \pm 32$ & $75 \pm 32$ & $78 \pm 32$ & 6.0 & 7.26 & 2 & 3.63 \\
\hline WISEA J214025.23-332707.4 & 325.1066145 & -33.4533963 & 23 & 25 & 58728.28 & $415 \pm 67$ & $-515 \pm 69$ & $661 \pm 69$ & 93.2 & 2.10 & 2 & 1.05 \\
\hline WISEU J215018.46-752053.0 & 327.5808368 & -75.3486640 & 40 & 18 & 58438.92 & $918 \pm 40$ & $-307 \pm 40$ & $968 \pm 40$ & 571.9 & 5.80 & 10 & 0.58 \\
\hline WISEAR J220746.67-503631.7 & 331.9449276 & -50.6122455 & 27 & 26 & 58503.19 & $108 \pm 99$ & $-1380 \pm 98$ & $1384 \pm 98$ & 198.1 & 4.40 & 10 & 0.44 \\
\hline
\end{tabular}


Table 6

(Continued)

\begin{tabular}{|c|c|c|c|c|c|c|c|c|c|c|c|c|}
\hline Name & $\begin{array}{c}\alpha_{0} \\
\text { (deg, ICRS) }\end{array}$ & $\begin{array}{c}\delta_{0} \\
(\mathrm{deg}, \mathrm{ICRS})\end{array}$ & $\begin{array}{l}\sigma_{\alpha 0} \\
\text { (mas) }\end{array}$ & $\begin{array}{c}\sigma_{\delta_{0}} \\
\text { (mas) }\end{array}$ & $\mathrm{MJD}_{0}$ & $\begin{array}{c}\mu_{\alpha} \\
\left(\operatorname{mas~yr}^{-1}\right)\end{array}$ & $\begin{array}{c}\mu_{\delta} \\
\left(\operatorname{mas~yr}^{-1}\right)\end{array}$ & $\begin{array}{c}\mu_{\mathrm{tot}} \\
\left(\operatorname{mas~yr}^{-1}\right)\end{array}$ & $\chi_{\text {motion }}^{2}$ & $\chi^{2}$ & dof & $\chi_{\nu}^{2}$ \\
\hline WISEA J221859.33+114644.4 & 334.7477539 & 11.7783302 & 11 & 20 & 58394.99 & $224 \pm 31$ & $-267 \pm 33$ & $349 \pm 32$ & 118.0 & 1.33 & 2 & 0.67 \\
\hline WISEA J221841.38+143003.4 & 334.6713695 & 14.5003718 & 14 & 29 & 58748.87 & $-387 \pm 33$ & $-307 \pm 34$ & $494 \pm 33$ & 218.4 & 3.67 & 10 & 0.37 \\
\hline WISEA J224319.56-145857.3 & 340.8321592 & -14.9839027 & 30 & 21 & 58396.41 & $286 \pm 49$ & $-541 \pm 50$ & $611 \pm 50$ & 150.2 & 0.51 & 2 & 0.25 \\
\hline WISEU J224547.11-433341.5 & 341.4481561 & -43.5620463 & 27 & 26 & 58490.27 & $1108 \pm 35$ & $-388 \pm 35$ & $1174 \pm 35$ & 1131.1 & 2.73 & 2 & 1.37 \\
\hline WISEA J225404.16-265257.5 & 343.5188443 & -26.8827008 & 24 & 43 & 58389.63 & $566 \pm 54$ & $-63 \pm 54$ & $569 \pm 54$ & 113.0 & 0.96 & 2 & 0.48 \\
\hline WISEA J230930.58+145633.1 & 347.3789785 & 14.9414978 & 41 & 34 & 58383.22 & $664 \pm 59$ & $-466 \pm 60$ & $811 \pm 59$ & 187.3 & 3.79 & 12 & 0.32 \\
\hline WISEA J233816.47-732929.7 & 354.5743186 & -73.4919335 & 19 & 22 & 58385.05 & $755 \pm 32$ & $-114 \pm 30$ & $764 \pm 31$ & 588.8 & 3.71 & 10 & 0.37 \\
\hline WISEA J235120.62-700025.8 & 357.8354396 & -70.0074316 & 25 & 43 & 58665.89 & $-101 \pm 37$ & $-139 \pm 36$ & $172 \pm 36$ & 22.3 & 0.42 & 2 & 0.21 \\
\hline WISEA J235456.63-481440.1 & 358.7372720 & -48.2450119 & 40 & 40 & 58373.02 & $403 \pm 50$ & $-231 \pm 50$ & $465 \pm 50$ & 85.7 & 3.21 & 2 & 1.60 \\
\hline
\end{tabular}

Note. $\chi_{\nu}^{2}=\chi^{2} /($ dof $)$.

(This table is available in machine-readable form.) 


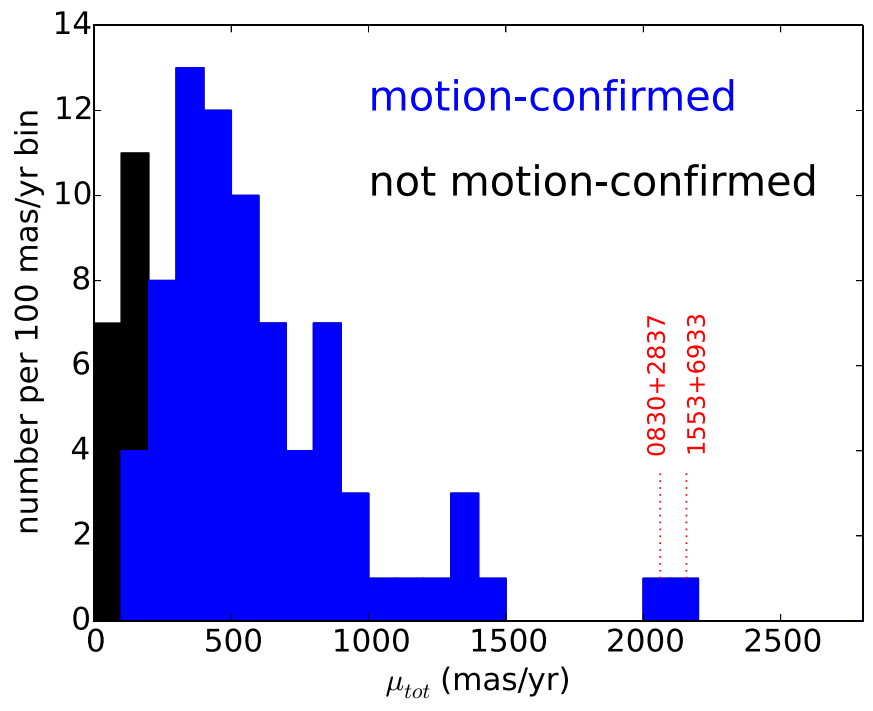

Figure 4. Distribution of best-fit total linear motions for our brown dwarf candidates. The number of sample members per bin that were not motion confirmed is shown in black, stacked on top of the number of motion-confirmed

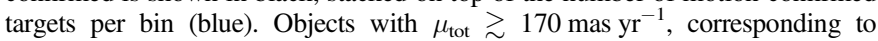
approximately half of a WISE pixel of displacement over the available time baseline, tend to be motion confirmed. One new discovery (WISEA 1553+6933) has best-fit $\mu_{\mathrm{tot}}>2000 \mathrm{mas} \mathrm{yr}^{-1}$, and eight have $\mu_{\mathrm{tot}}>1000 \mathrm{mas} \mathrm{yr}^{-1}$; these number counts do not include WISEA $0830+2837\left(\mu_{\text {tot }} \approx 2060\right.$ mas yr $\left.^{-1}\right)$, since this object's discovery was previously presented in Bardalez Gagliuffi et al. (2020).

appropriate zero-point. The $5 \sigma$ depths ranged from 15.7 to $19.2 \mathrm{mag}$, with a median value of $18.1 \mathrm{mag}$.

\subsection{Archival Near-infrared Photometry}

We have also compiled archival NIR photometry for members of our sample using the WFCAM Science Archive (UKIRT/ WFCAM data; Hambly et al. 2008) and VISTA Science Archive (VISTA/VIRCAM data; Cross et al. 2012). We issued a cone search in the vicinity of each target and visually vetted possible NIR counterparts to avoid spurious positional crossmatches. We searched for archival NIR photometry in the $Y, J, H$, and $K$ bands ( $K_{S}$ rather than $K$ in the case of VIRCAM). The NIR counterparts are predominantly drawn from the UKIRT Hemisphere Survey in the north (UHS; Dye et al. 2018) and the VISTA Hemisphere Survey in the south (VHS; McMahon et al. 2013), though VIKING (Edge et al. 2013) and the UKIRT Infrared Deep Sky Survey (Lawrence et al. 2007) also contribute. By selection, all members of our sample lack 2MASS (Skrutskie et al. 2006) counterparts.

\subsection{Merged Near-infrared Photometry}

Table 7 provides a merged compilation of OMM and archival NIR photometry for motion-confirmed targets, in the $Y J H K / K_{S}$ bands. In cases where both OMM and archival follow-up $J$-band photometry are available, Table 7 quotes the higher significance measurement. Table 8 reports additional OMM follow-up results for targets that are not motion confirmed and/or have higher-significance $J$-band measurements in archival data sets. Our follow-up OMM photometry agrees well with archival photometry in cases where both are available.

Figure 5 shows a color-color plot of $J-\operatorname{ch} 2$ versus $\operatorname{ch} 1-\operatorname{ch} 2$ for motion-confirmed targets with both colors available. The data points are by and large in good agreement with the expected trend for brown dwarfs (Dupuy \& Liu 2012), providing further assurance that our motion confirmation process has weeded out static contaminants that might display rather discrepant colors. WISEAR 2207-5036, a suspected subdwarf owing to its high kinematics (Section 11.5.1), stands out as likely the strongest color outlier. Even if all other sample members are typical field brown dwarfs, we would statistically expect some fraction to scatter $>1 \sigma$ off of the Dupuy \& Liu (2012) locus; this likely explains most or all of the Figure 5 $J$-band detections falling modestly outside of the purple shaded region. Analogous color-color diagrams for $Y-\operatorname{ch} 2, H-\operatorname{ch} 2$, and $K$-ch2 again show agreement with the expected trends for brown dwarfs, though we have omitted those plots owing to their paucity of NIR detections.

In addition to the ground-based NIR photometry gathered here, five of our targets have HST follow-up NIR photometry (Bardalez Gagliuffi et al. 2020). Owing to the nonstandard bandpasses of these HST data, we have chosen not to merge the HST measurements with our ground-based photometry in this work.

\section{Results and Discussion}

\subsection{Derived Parameters}

Table 9 lists various properties that we are able to derive based on our ch1 and ch2 magnitudes, for the sample of motion-confirmed objects with both of these Spitzer photometric data points available. ${ }^{23}$ These derived parameters are as follows:

1. Phototype: We estimate phototypes using the Kirkpatrick et al. (2019) relation for spectral type as a function of ch1 -ch2 color. We use the same phototyping procedure as Meisner et al. (2020), which contains a more detailed explanation of our methodology and associated caveats. In brief, Meisner et al. (2020) estimate an rms phototyping uncertainty of approximately \pm 1 subtype. Larger errors between phototype and real spectral type for individual objects are of course possible, and photometric type estimates should never be considered substitutes for true spectral types. For the four objects successfully assigned a true spectral type and subtype in Table 3, our phototypes achieve the expected level of agreement: an rms difference of 1.03 subtypes.

2. Absolute ch2 magnitude: We estimate the absolute ch2 magnitude using the Kirkpatrick et al. (2019) relation for $M_{\mathrm{ch} 2}$ as a function of ch1-ch2. Again, our procedure is the same as that of Meisner et al. (2020), which contains further details, particularly in relation to the quoted uncertainties.

3. Distance: Photometric distance estimates for members of our sample follow from the combination of each object's measured apparent ch2 magnitude and $M_{\mathrm{ch} 2}$ estimate. Details of how we quote these distances and their associated uncertainties can be found in Meisner et al. (2020).

4. Effective temperature: We provide $T_{\text {eff }}$ estimates based on the Kirkpatrick et al. (2019) relation for effective

\footnotetext{
23 WISEA $0535-6445$ is excluded from Table 9 despite having a Spitzer color measurement, since its $\operatorname{ch} 1-\operatorname{ch} 2 \approx 0$ color does not place a strong constraint on its spectral type or absolute magnitude. See Section 11.7.2 for further discussion of this object.
} 
Table 7

Ground-based NIR Photometry from the VISTA/UKIRT Archives and OMM Follow-up for Motion-confirmed Members of Our Sample

\begin{tabular}{|c|c|c|c|c|c|c|}
\hline Name & $\begin{array}{c}Y \\
(\mathrm{mag})\end{array}$ & $\begin{array}{l}J_{\mathrm{MKO}} \\
(\mathrm{mag})\end{array}$ & $\begin{array}{c}H_{\mathrm{MKO}} \\
(\mathrm{mag})\end{array}$ & $\begin{array}{l}K_{\mathrm{MKO}} \\
(\mathrm{mag})\end{array}$ & $\begin{array}{c}K_{S} \\
(\mathrm{mag})\end{array}$ & Origin \\
\hline WISEA J001449.96+795116.1 & $\cdots$ & $19.72 \pm 0.62$ & $\cdots$ & $\cdots$ & $\cdots$ & CPAPIR \\
\hline WISEU J001908.31-094323.3 & $19.72 \pm 0.10$ & $18.80 \pm 0.08$ & $\cdots$ & $\cdots$ & $>18.13$ & VIRCAM \\
\hline WISEA J002810.59+521853.1 & $\cdots$ & $19.20 \pm 0.16$ & $\cdots$ & $\cdots$ & $\ldots$ & WFCAM \\
\hline WISEA J002909.90+190516.6 & $\cdots$ & $17.81 \pm 0.04$ & $\cdots$ & $\cdots$ & $\cdots$ & WFCAM \\
\hline WISEU J004851.21+250814.9 & $\cdots$ & $19.51 \pm 0.16$ & $\cdots$ & $\cdots$ & $\cdots$ & WFCAM \\
\hline WISEU J005559.88+594745.0 & $\cdots$ & $17.90 \pm 0.05$ & $\cdots$ & $\cdots$ & $\cdots$ & WFCAM \\
\hline WISEA J010116.12-520629.8 & $\cdots$ & $19.76 \pm 0.12$ & $\ldots$ & $\cdots$ & $>18.31$ & VIRCAM \\
\hline WISEA J012057.06-764216.4 & $20.14 \pm 0.14$ & $19.10 \pm 0.10$ & $\cdots$ & $\cdots$ & $>18.13$ & VIRCAM \\
\hline WISEA J012834.87-280302.5 & $18.86 \pm 0.02$ & $17.73 \pm 0.01$ & $17.88 \pm 0.03$ & $\cdots$ & $17.96 \pm 0.06$ & VIRCAM \\
\hline WISEA J013217.78-581825.9 & $\ldots$ & $19.59 \pm 0.10$ & $\ldots$ & $\cdots$ & $>18.57$ & VIRCAM \\
\hline WISEU J013522.46-221957.3 & $\cdots$ & $18.24 \pm 0.03$ & $\cdots$ & $\cdots$ & $18.58 \pm 0.27$ & VIRCAM \\
\hline WISEA J021420.18-573245.1 & $\cdots$ & $19.73 \pm 0.18$ & $\cdots$ & $\cdots$ & $>18.03$ & VIRCAM \\
\hline WISEA J025805.29-321917.4 & $18.48 \pm 0.02$ & $17.72 \pm 0.01$ & $17.86 \pm 0.05$ & $\cdots$ & $17.38 \pm 0.05$ & VIRCAM \\
\hline WISEA J030534.09-582635.5 & $\cdots$ & $19.10 \pm 0.06$ & $\cdots$ & $\cdots$ & $>18.44$ & VIRCAM \\
\hline WISEA J032600.28+421056.8 & $\cdots$ & $18.71 \pm 0.12$ & $\cdots$ & $\cdots$ & $\cdots$ & WFCAM \\
\hline WISEA J032811.00-422321.6 & $\cdots$ & $19.16 \pm 0.09$ & $\cdots$ & $\cdots$ & $\cdots$ & VIRCAM \\
\hline WISEA J034227.44-462252.0 & $\cdots$ & $18.73 \pm 0.06$ & $19.15 \pm 0.19$ & $\cdots$ & $>18.44$ & VIRCAM \\
\hline WISEA J035733.85+070557.4 & $\cdots$ & $17.66 \pm 0.05$ & $\ldots$ & $\cdots$ & $\ldots$ & WFCAM \\
\hline WISEA J040702.42+190945.8 & $\cdots$ & $17.73 \pm 0.04$ & $\cdots$ & $18.20 \pm 0.18$ & $\cdots$ & WFCAM \\
\hline WISEA J042236.95-044203.5 & $>19.97$ & $19.43 \pm 0.23$ & $\cdots$ & $\cdots$ & $>18.89$ & VIRCAM \\
\hline WISEA J050238.28+100750.0 & $\cdots$ & $18.90 \pm 0.11$ & $\cdots$ & $\cdots$ & $\cdots$ & WFCAM \\
\hline WISEU J050305.68-564834.0 & $\cdots$ & $>20.29$ & $\cdots$ & $\cdots$ & $>18.42$ & VIRCAM \\
\hline WISEA J050615.56-514521.3 & $\cdots$ & $>20.05$ & $>19.01$ & $\cdots$ & $>18.30$ & VIRCAM \\
\hline WISEA J053512.01-773829.7 & $>20.31$ & $19.67 \pm 0.20$ & $\cdots$ & $\cdots$ & $>18.05$ & VIRCAM \\
\hline WISEA J064503.72+524054.1 & $\ldots$ & $19.96 \pm 0.22$ & $\cdots$ & $\cdots$ & $\ldots$ & WFCAM \\
\hline WISEA J065113.90-835502.6 & $\cdots$ & $19.39 \pm 0.19$ & $\cdots$ & $\cdots$ & $>17.92$ & VIRCAM \\
\hline WISEA J075438.20+090044.9 & $\cdots$ & $19.63 \pm 0.21$ & $\cdots$ & $\cdots$ & $\cdots$ & WFCAM \\
\hline WISEA J080622.22-082046.5 & $\cdots$ & $>19.60$ & $\cdots$ & $\cdots$ & $>18.07$ & VIRCAM \\
\hline WISEA J083011.95+283716.0 & $\cdots$ & $>18.57$ & $\cdots$ & $\cdots$ & $\cdots$ & CPAPIR \\
\hline WISEA J083019.97-632305.4 & $\cdots$ & $18.64 \pm 0.10$ & $\cdots$ & $\cdots$ & $\cdots$ & VIRCAM \\
\hline WISEA J101804.20-684254.0 & $\cdots$ & $18.58 \pm 0.09$ & $\cdots$ & $\cdots$ & $>17.74$ & VIRCAM \\
\hline WISEA J104216.89-003935.9 & $20.09 \pm 0.07$ & $19.18 \pm 0.04$ & $19.41 \pm 0.11$ & $\cdots$ & $\cdots$ & VIRCAM \\
\hline WISEA J105349.41-460241.2 & $\cdots$ & $18.64 \pm 0.10$ & $\ldots$ & $\cdots$ & $>17.86$ & VIRCAM \\
\hline WISEA J105917.38+285729.3 & $\cdots$ & $18.61 \pm 0.09$ & $\cdots$ & $\cdots$ & $\ldots$ & WFCAM \\
\hline WISEA J110201.76+350335.4 & $\cdots$ & $19.12 \pm 0.13$ & $\cdots$ & $\cdots$ & $\cdots$ & WFCAM \\
\hline WISEA J112440.19+663052.0 & $\cdots$ & $18.48 \pm 0.30$ & $\cdots$ & $\cdots$ & $\cdots$ & CPAPIR \\
\hline WISEA J114350.90+401333.9 & $\cdots$ & $18.52 \pm 0.07$ & $\cdots$ & $\cdots$ & $\cdots$ & WFCAM \\
\hline WISEA J114601.22+342458.8 & $\cdots$ & $18.09 \pm 0.04$ & $\cdots$ & $\cdots$ & $\cdots$ & WFCAM \\
\hline WISEA J115917.89+671704.2 & $\cdots$ & $>18.40$ & $\cdots$ & $\cdots$ & $\cdots$ & CPAPIR \\
\hline WISEA J125721.01+715349.3 & $\cdots$ & $>19.02$ & $\cdots$ & $\cdots$ & $\cdots$ & CPAPIR \\
\hline WISEA J143422.31-083934.2 & $>20.04$ & $>19.55$ & $>19.51$ & $\cdots$ & $>18.29$ & VIRCAM \\
\hline WISEA J151620.39+721745.4 & $\ldots$ & $20.34 \pm 0.66$ & $\ldots$ & $\cdots$ & $\ldots$ & CPAPIR \\
\hline WISEA J152529.09+605356.5 & $\cdots$ & $>18.34$ & $\cdots$ & $\cdots$ & $\cdots$ & CPAPIR \\
\hline WISEAR J154025.77-113940.8 & $20.28 \pm 0.26$ & $19.57 \pm 0.20$ & $>19.29$ & $\cdots$ & $>18.79$ & VIRCAM \\
\hline WISEA J155349.96+693355.2 & $\ldots$ & $>17.34$ & $\ldots$ & $\cdots$ & $\ldots$ & CPAPIR \\
\hline WISEA J160516.79+002139.0 & $\cdots$ & $19.02 \pm 0.12$ & $\cdots$ & $\cdots$ & $\cdots$ & WFCAM \\
\hline WISEA J162852.64+160421.0 & $\cdots$ & $>19.50$ & $\cdots$ & $\cdots$ & $\cdots$ & WFCAM \\
\hline WISEA J163932.75+184049.4 & $\cdots$ & $>19.47$ & $\cdots$ & $\cdots$ & $\cdots$ & WFCAM \\
\hline WISEA J171331.68+245000.9 & $\cdots$ & $19.37 \pm 0.16$ & $\cdots$ & $\cdots$ & $\cdots$ & WFCAM \\
\hline WISEA J172907.10-753017.0 & $\cdots$ & $17.80 \pm 0.04$ & $\cdots$ & $\cdots$ & $18.18 \pm 0.27$ & VIRCAM \\
\hline WISEA J175328.55-590447.6 & $\cdots$ & $19.05 \pm 0.09$ & $\cdots$ & $\cdots$ & $>18.02$ & VIRCAM \\
\hline WISEA J181849.59-470146.9 & $\cdots$ & $19.81 \pm 0.22$ & $\cdots$ & $\cdots$ & $>18.12$ & VIRCAM \\
\hline WISEA J190005.76-310810.9 & $\cdots$ & $18.40 \pm 0.06$ & $\cdots$ & $\cdots$ & $>18.16$ & VIRCAM \\
\hline WISEA J193054.55-205949.4 & $\cdots$ & $>19.80$ & $\cdots$ & $\cdots$ & $>18.08$ & VIRCAM \\
\hline WISEA J201833.67-141720.3 & $\cdots$ & $19.13 \pm 0.00$ & $\cdots$ & $\cdots$ & $>18.28$ & VIRCAM \\
\hline WISEA J205921.85+662725.2 & $\cdots$ & $>16.07$ & $\cdots$ & $\cdots$ & $\cdots$ & CPAPIR \\
\hline WISEA J211456.86-180519.0 & $19.46 \pm 0.08$ & $18.34 \pm 0.06$ & $\cdots$ & $\cdots$ & $>18.09$ & VIRCAM \\
\hline WISEA J212510.91-730758.8 & $19.58 \pm 0.16$ & $18.76 \pm 0.14$ & $\cdots$ & $\cdots$ & $>17.79$ & VIRCAM \\
\hline WISEA J214025.23-332707.4 & $>20.50$ & $19.96 \pm 0.26$ & $\cdots$ & $\ldots$ & $>18.11$ & VIRCAM \\
\hline WISEU J215018.46-752053.0 & $18.53 \pm 0.13$ & $18.10 \pm 0.10$ & $\cdots$ & $\cdots$ & $>17.40$ & VIRCAM \\
\hline WISEAR J220746.67-503631.7 & $\cdots$ & $>20.36$ & $>18.98$ & $\cdots$ & $>18.38$ & VIRCAM \\
\hline WISEA J221859.33+114644.4 & $\cdots$ & $17.94 \pm 0.06$ & $\cdots$ & $\cdots$ & $\cdots$ & WFCAM \\
\hline WISEA J221841.38+143003.4 & $\cdots$ & $19.14 \pm 0.12$ & $\cdots$ & $\cdots$ & $\cdots$ & WFCAM \\
\hline
\end{tabular}


Table 7

(Continued)

\begin{tabular}{|c|c|c|c|c|c|c|}
\hline Name & $\begin{array}{c}Y \\
(\mathrm{mag})\end{array}$ & $\begin{array}{l}J_{\mathrm{MKO}} \\
(\mathrm{mag})\end{array}$ & $\begin{array}{c}H_{\mathrm{MKO}} \\
(\mathrm{mag})\end{array}$ & $\begin{array}{l}K_{\mathrm{MKO}} \\
(\mathrm{mag})\end{array}$ & $\begin{array}{c}K_{S} \\
(\mathrm{mag})\end{array}$ & Origin \\
\hline WISEA J224319.56-145857.3 & $>20.43$ & $>19.91$ & $\cdots$ & $\cdots$ & $>18.06$ & VIRCAM \\
\hline WISEU J224547.11-433341.5 & $\ldots$ & $18.10 \pm 0.03$ & $\cdots$ & $\cdots$ & $18.53 \pm 0.29$ & VIRCAM \\
\hline WISEA J230930.58+145633.1 & $\ldots$ & $17.75 \pm 0.04$ & $\cdots$ & $\cdots$ & $\cdots$ & WFCAM \\
\hline WISEA J233816.47-732929.7 & $20.39 \pm 0.18$ & $19.30 \pm 0.13$ & $\cdots$ & $\cdots$ & $>18.03$ & VIRCAM \\
\hline WISEA J235456.63-481440.1 & $\ldots$ & $>20.11$ & $>19.33$ & $\cdots$ & $>18.61$ & VIRCAM \\
\hline
\end{tabular}

Note. Magnitude limits are $5 \sigma$.

(This table is available in machine-readable form.)

Table 8

Additional OMM $J$-band Photometry

\begin{tabular}{|c|c|}
\hline Designation & $\begin{array}{l}J_{\mathrm{MKO}} \\
(\mathrm{mag})\end{array}$ \\
\hline WISEA J002810.59+521853.1 & $19.58 \pm 0.58$ \\
\hline WISEA J002909.90+190516.6 & $18.02 \pm 0.17$ \\
\hline WISEU J005559.88+594745.0 & $16.52 \pm 0.05^{\mathrm{a}}$ \\
\hline WISEA J032600.28+421056.8 & $18.85 \pm 0.28$ \\
\hline WISEA J035733.85+070557.4 & $17.51 \pm 0.08$ \\
\hline WISEA J040702.42+190945.8 & $17.61 \pm 0.08$ \\
\hline WISEA J042236.95-044203.5 & $19.62 \pm 0.39$ \\
\hline WISEA J050238.28+100750.0 & $>16.84$ \\
\hline WISEA J064503.72+524054.1 & $19.82 \pm 0.68$ \\
\hline WISEA J075438.20+090044.9 & $>17.95$ \\
\hline WISEA J080622.22-082046.5 & $>16.64$ \\
\hline WISEA J104051.77+450329.3 & $18.04 \pm 0.08$ \\
\hline WISEA J104216.89-003935.9 & $19.47 \pm 0.40$ \\
\hline WISEA J105917.38+285729.3 & $18.39 \pm 0.28$ \\
\hline WISEA J110201.76+350335.4 & $19.56 \pm 0.82$ \\
\hline WISEA J114350.90+401333.9 & $18.82 \pm 0.27$ \\
\hline WISEA J114601.22+342458.8 & $18.29 \pm 0.10$ \\
\hline WISEA J143422.31-083934.2 & $>18.12$ \\
\hline WISEAR J154025.77-113940.8 & $>18.08$ \\
\hline WISEA J161940.51+134752.0 & $>18.07$ \\
\hline WISEA J162716.41-244355.4 & $>15.73$ \\
\hline WISEA J162852.64+160421.0 & $>18.32$ \\
\hline WISEA J163932.75+184049.4 & $>18.28$ \\
\hline WISEA J171331.68+245000.9 & $19.36 \pm 0.25$ \\
\hline WISEA J175701.50+183215.2 & $>17.39$ \\
\hline WISEA J185109.93+671343.7 & $>18.79$ \\
\hline WISEA J193054.55-205949.4 & $>17.01$ \\
\hline WISEA J201833.67-141720.3 & $19.45 \pm 0.66$ \\
\hline WISEA J211456.86-180519.0 & $18.45 \pm 0.27$ \\
\hline WISEA J213810.99+373312.9 & $>16.01$ \\
\hline WISEA J221841.38+143003.4 & $>17.78$ \\
\hline WISEA J224319.56-145857.3 & $>17.83$ \\
\hline WISEA J230930.58+145633.1 & $17.80 \pm 0.12$ \\
\hline
\end{tabular}

Notes. This table reports follow-up $J$ band photometry for targets not motion confirmed and/or with higher-significance $J$-band measurements from archival data sets. Magnitude limits are $5 \sigma$.

a The OMM photometry for WISEU $0055+5947$ is contaminated by blending with a brighter nearby source, resulting in a reported magnitude that is artificially bright.

(This table is available in machine-readable form.)

temperature as a function of ch1-ch 2 color. No $T_{\text {eff }}$ value is listed if ch1-ch2 $<0.9$, since the Kirkpatrick et al. (2019) $T_{\text {eff }}(\operatorname{ch} 1-\operatorname{ch} 2)$ relation is not applicable in that Spitzer color regime.
5. Tangential velocity: $V_{\tan }$ estimates follow from the combination of our $\mu_{\text {tot }}$ values in Table 6 and our photometric distance estimates. As shown in Figure 6, our set of targets appears to have a higher typical $V_{\tan }$ value than that found for the Kirkpatrick et al. (2019) volume-limited sample of mid- to late $\mathrm{T}$ and $\mathrm{Y}$ dwarfs within 20 pc. The 180 Kirkpatrick et al. (2019) objects within $20 \mathrm{pc}$ have a median $V_{\tan }$ of $35 \mathrm{~km} \mathrm{~s}^{-1}$, whereas our sample's median $V_{\tan }$ is $60 \mathrm{~km} \mathrm{~s}^{-1}$. This presumably owes to a bias whereby our motion-selected sample preferentially contains relatively faint and fast-moving targets.

Table 9 also includes ch2 reduced proper motions, ${ }^{24}$ calculated by combining linear motion fitting results from Table 6 with the apparent ch2 magnitudes from Table 2. Figure 6 shows histograms of $\operatorname{ch} 1-\mathrm{ch} 2$ color, photometric distance, and $V_{\tan }$ for objects in Table 9. Further discussion subsections draw heavily on the Table 9 parameters to highlight notable aspects and members of our sample.

\subsection{The 10 and 20 pc Samples}

Studies of the substellar mass function are currently limited by incompleteness of the local $(\lesssim 20 \mathrm{pc})$ brown dwarf sample at the latest spectral types (Kirkpatrick et al. 2019). A core objective of this work was therefore to help pinpoint previously unidentified members of the 20 pc census.

In terms of our nearest motion-confirmed discoveries, two have best-fit photometric distance estimates (Table 9) within 10 pc: WISEA $0830+2837$ and WISEA 1930-2059. WISEA 1930-2059 also has a $1 \sigma$ distance upper envelope value within 10 pc. For WISEA $0830+2837$, Bardalez Gagliuffi et al. (2020) have published a WISE+Spitzer trigonometric parallax of $90.6 \pm 13.7$ mas, placing this object most likely outside of the $10 \mathrm{pc}$ sample, though still possibly closer than $10 \mathrm{pc}$ within the $1 \sigma$ parallax uncertainty. WISEU $0503-5648$ has a central distance estimate larger than $10 \mathrm{pc}$ but could still be closer than $10 \mathrm{pc}$ within its $1 \sigma$ photometric distance estimate uncertainty.

Sixteen of our motion-confirmed discoveries have central photometric distance estimates within $20 \mathrm{pc}$. An additional 15 of our motion-confirmed discoveries have central photometric distance estimates larger than $20 \mathrm{pc}$ but still within $1 \sigma$ of the 20 pc threshold. Considering that the Kirkpatrick et al. (2019) 20 pc sample of late $\mathrm{T}$ and $\mathrm{Y}$ dwarfs consisted of 235 objects (46 of which have central parallax values placing them within 10

${ }^{24}$ Defined as $H_{\mathrm{ch} 2}=m_{\mathrm{ch} 2}+5 \log _{10} \mu+5$. 


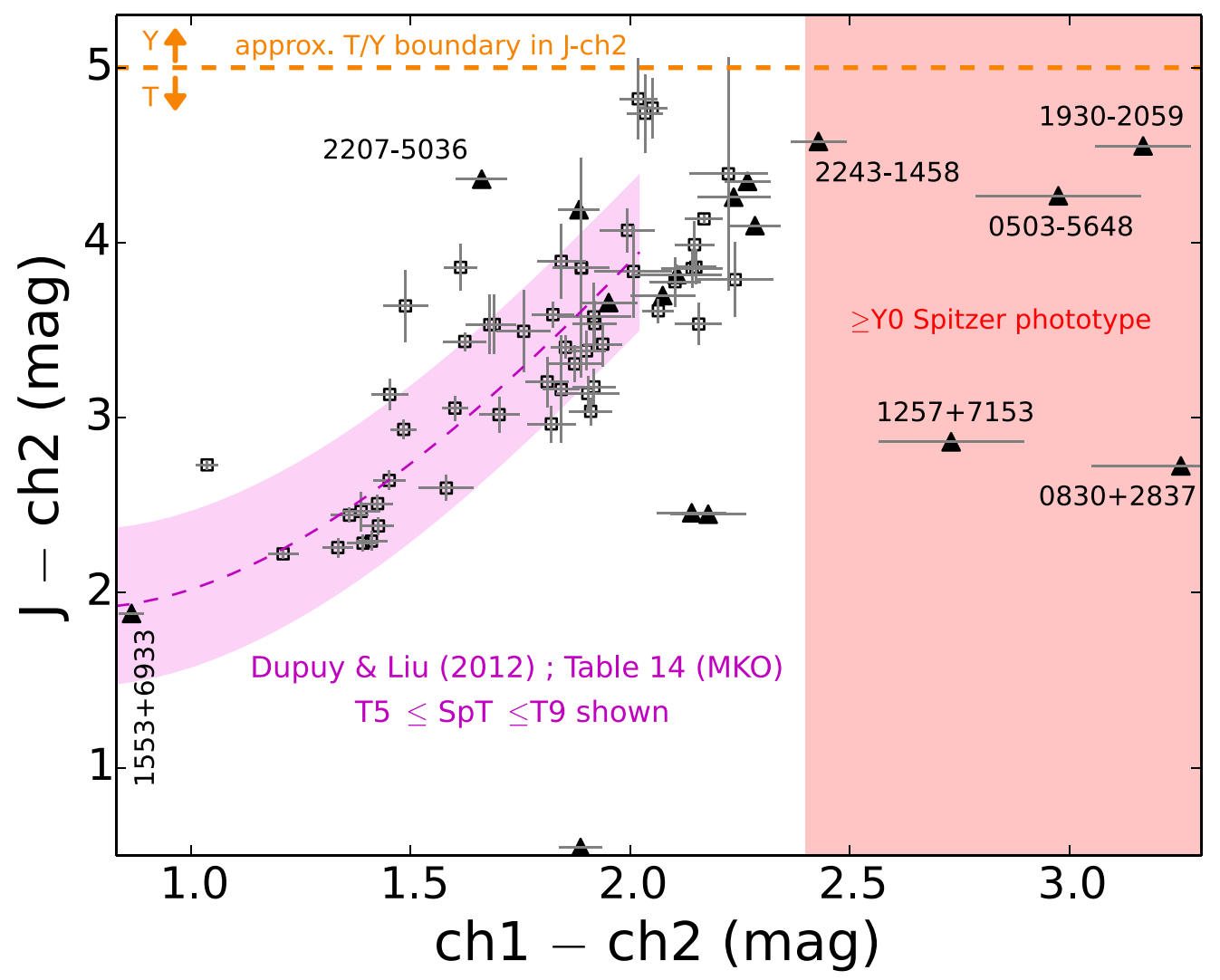

Figure 5. $J$-ch2 vs. ch1-ch2 for motion-confirmed targets with both colors available. Follow-up $J$-band photometry comes from OMM/CPAPIR, and archival $J$ band photometry is obtained from the WFCAM/VISTA archives. Black squares represent detections. All $J$-ch2 lower limits (black triangles) are based on $5 \sigma J$ limits. The dashed purple line shows the relation for mid- to late T dwarfs from Dupuy \& Liu (2012), with the purple shaded region indicating the $1 \sigma$ scatter in that relation. Our motion-confirmed sample generally follows the expected trend. The light-red shaded region denotes the Spitzer color range within which our phototyping procedure yields $\geqslant \mathrm{Y} 0$ estimates, ch1-ch2 $\geqslant 2.4 \mathrm{mag}$. The dashed orange horizontal line indicates the approximate boundary between late $\mathrm{T}$ and early $\mathrm{Y}$ dwarfs in terms of $J-\operatorname{ch} 2$ color. Our $J$-ch2 limit for WISEAR 2207-5036, a suspected subdwarf owing to its high kinematics, indicates that it is also a color outlier.

pc), our discoveries represent a sizable new batch of candidate 20 pc sample members.

\subsection{Y Dwarf Candidates}

Given our use of the Kirkpatrick et al. (2019) phototyping relation, we have effectively placed our threshold for Y dwarf candidacy at ch1-ch2 > $2.4 \mathrm{mag}$. Five of our motionconfirmed brown dwarf candidates have best-fit Spitzer colors most consistent with $\geqslant Y 0$ phototypes: WISEU 0503-5648, WISEA $0830+2837$, WISEA $1257+7153$, WISEA 1930 -2059, and WISEA 2243-1458. WISEA $0830+2837$ has already been discussed thoroughly in Bardalez Gagliuffi et al. (2020) and is thought to be of type $\geqslant Y 1$. Of the other four motion-confirmed $\mathrm{Y}$ dwarf candidates first presented in this work, three have exceptionally red Spitzer colors most consistent with types $\geqslant \mathrm{Y} 1$. This new crop of $\geqslant \mathrm{Y} 1$ candidates discovered by Backyard Worlds is particularly significant given that only four to six spectroscopically confirmed brown dwarfs are currently known in this regime: WISE 0350-5658 (Y1; Kirkpatrick et al. 2012), WISE 0647-6232 (Y1; Kirkpatrick et al. 2012), WISE 1541-2250 (Y1; Cushing et al. 2011), WISE 2354-0240 (Y1; Schneider et al. 2015), WISE 1828 +2650 ( $\geqslant$ Y2 with highly uncertain $T_{\text {eff }}$; Beichman et al. 2013), and WISE 0855-0714 ( $L$ - and $M$-band spectra; Skemer et al. 2016; Morley et al. 2018). The impact of our sample's likely coldest four discoveries in terms of mass function analyses is also considerable: only nine previously known objects in the Kirkpatrick et al. (2019) 20 pc compilation inhabit the corresponding $T_{\text {eff }}<400 \mathrm{~K}$ effective temperature range.

Of our five motion-confirmed discoveries with phototypes $\geqslant \mathrm{Y} 0$, four are more than $2 \sigma$ redder than our $\mathrm{T} / \mathrm{Y}$ threshold color of ch1 $-\mathrm{ch} 2=2.4 \mathrm{mag}$. On the other hand, WISEA 2243 -1458 is within its $1 \sigma$ color uncertainty of being classified as a late $\mathrm{T}$ dwarf. A sixth member of our sample (WISEA 2351 -7000 ) would have a Y phototype based on its ch1-ch2 color, but it falls just short of exceeding our $\chi_{\text {motion }}^{2}$ significance threshold (Table 6). Figure 7 shows WISE+Spitzer motion trajectories for all six targets with ch1-ch2 > 2.4 mag, including WISEA 2351-7000. The WISEA 2351-7000 WISE + Spitzer astrometric trajectory looks plausibly linear, but more data will be needed to conclusively establish whether this source is moving.

Each of our five motion-confirmed $\mathrm{Y}$ dwarf candidates is labeled in Figure 5. Clearly, the existing $J$ limits for these sources are not deep enough to confirm that their $J-\operatorname{ch} 2$ colors are securely in the $J-\operatorname{ch} 2 \gtrsim 5$ mag regime occupied by Y dwarfs. Therefore, deeper ground-based NIR imaging of our Y dwarf candidates would be of high value, with the exception of WISEA $0830+2837$, which already has extreme F125W - ch2 > 9.36 mag and F105W - ch2 > 9.56 mag limits available (Bardalez Gagliuffi et al. 2020). 
Table 9

Derived Parameters of Motion-confirmed Objects with Spitzer ch1 and ch2 Photometry

\begin{tabular}{|c|c|c|c|c|c|c|}
\hline Name & $\begin{array}{c}\text { SpT } \\
\text { (phototype) }\end{array}$ & $\begin{array}{l}M_{\mathrm{ch} 2} \\
(\mathrm{mag})\end{array}$ & $\begin{array}{l}\text { Distance } \\
\quad(\mathrm{pc})\end{array}$ & $\begin{array}{l}T_{\text {eff }} \\
(\mathrm{K})\end{array}$ & $\begin{array}{c}H_{\mathrm{ch} 2} \\
(\mathrm{mag})\end{array}$ & $\begin{array}{c}V_{\tan } \\
\left(\mathrm{km} \mathrm{s}^{-1}\right)\end{array}$ \\
\hline CWISE J000229.93+635217.0 & 7.5 & $13.41_{-0.39}^{+0.42}$ & $28.6_{-5.1}^{+5.6}$ & $730_{-124}^{+132}$ & $20.21_{-0.14}^{+0.15}$ & $109_{-21}^{+22}$ \\
\hline WISEA J001449.96+795116.1 & 8.0 & $13.68_{-0.31}^{+0.31}$ & $27.4_{-3.7}^{+4.2}$ & $643_{-84}^{+84}$ & $19.30_{-0.19}^{+0.21}$ & $63_{-10}^{+11}$ \\
\hline WISEU J001908.31-094323.3 & 7.0 & $13.20_{-0.30}^{+0.30}$ & $31.1_{-4.0}^{+4.6}$ & $815_{-83}^{+83}$ & $18.14_{-0.37}^{+0.45}$ & $46_{-10}^{+11}$ \\
\hline WISEA J002810.59+521853.1 & 7.5 & $13.44_{-0.30}^{+0.30}$ & $27.9_{-3.7}^{+4.2}$ & $718_{-83}^{+83}$ & $18.99_{-0.16}^{+0.17}$ & $61_{-9}^{+10}$ \\
\hline WISEA J002909.90+190516.6 & 7.0 & $13.18_{-0.30}^{+0.30}$ & $26.6_{-3.4}^{+4.0}$ & $827_{-82}^{+82}$ & $17.73_{-0.27}^{+0.30}$ & $39_{-7}^{+8}$ \\
\hline WISEU J004851.21+250814.9 & 8.5 & $13.89_{-0.30}^{+0.30}$ & $14.8_{-1.9}^{+2.2}$ & $588_{-82}^{+82}$ & $20.22_{-0.04}^{+0.04}$ & $87_{-11}^{+13}$ \\
\hline WISEU J005559.88+594745.0 & 7.0 & $13.23_{-0.30}^{+0.30}$ & $22.2_{-2.9}^{+3.3}$ & $801_{-82}^{+82}$ & $18.02_{-0.13}^{+0.14}$ & $43_{-6}^{+7}$ \\
\hline WISEA J010116.12-520629.8 & 8.5 & $13.82_{-0.31}^{+0.31}$ & $23.7_{-3.2}^{+3.7}$ & $607_{-83}^{+84}$ & $19.96_{-0.13}^{+0.14}$ & $80_{-12}^{+13}$ \\
\hline WISEA J012057.06-764216.4 & 8.0 & $13.66_{-0.31}^{+0.31}$ & $26.7_{-3.6}^{+4.1}$ & $649_{-84}^{+84}$ & $18.21_{-0.27}^{+0.31}$ & $39_{-7}^{+8}$ \\
\hline WISEA J012834.87-280302.5 & 6.0 & $13.02_{-0.30}^{+0.30}$ & $31.5_{-4.1}^{+4.7}$ & $923_{-82}^{+82}$ & $17.51_{-0.30}^{+0.35}$ & $38_{-7}^{+8}$ \\
\hline WISEA J013217.78-581825.9 & 9.0 & $14.03_{-0.32}^{+0.32}$ & $22.0_{-3.0}^{+3.5}$ & $558_{-84}^{+84}$ & $18.96_{-0.20}^{+0.22}$ & $46_{-8}^{+8}$ \\
\hline WISEU J013522.46-221957.3 & 6.5 & $13.13_{-0.30}^{+0.30}$ & $34.1_{-4.4}^{+5.1}$ & $855_{-83}^{+83}$ & $18.66_{-0.23}^{+0.26}$ & $61_{-10}^{+11}$ \\
\hline WISEA J013810.78-412220.2 & 6.0 & $13.03_{-0.30}^{+0.30}$ & $42.9_{-5.6}^{+6.4}$ & $911_{-84}^{+84}$ & $20.28_{-0.26}^{+0.29}$ & $133_{-24}^{+26}$ \\
\hline WISEA J014603.23-261908.7 & 7.5 & $13.43_{-0.31}^{+0.31}$ & $29.9_{-3.9}^{+4.5}$ & $722_{-83}^{+84}$ & $19.79_{-0.19}^{+0.21}$ & $89_{-14}^{+16}$ \\
\hline WISEA J015507.35-421635.7 & 9.0 & $14.13_{-0.34}^{+0.34}$ & $25.5_{-3.7}^{+4.3}$ & $537_{-87}^{+87}$ & $19.63_{-0.23}^{+0.26}$ & $60_{-11}^{+12}$ \\
\hline WISEA J021420.18-573245.1 & 8.5 & $13.71_{-0.32}^{+0.32}$ & $30.8_{-4.2}^{+4.9}$ & $633_{-86}^{+86}$ & $19.78_{-0.21}^{+0.24}$ & $77_{-13}^{+15}$ \\
\hline WISEA J025756.40-265528.8 & 9.5 & $14.35_{-0.34}^{+0.34}$ & $20.9_{-3.1}^{+3.6}$ & $496_{-86}^{+86}$ & $19.65_{-0.21}^{+0.24}$ & $54_{-10}^{+11}$ \\
\hline WISEA J025805.29-321917.4 & 5.5 & $12.92_{-0.30}^{+0.30}$ & $25.9_{-3.4}^{+3.9}$ & $1006_{-82}^{+82}$ & $16.66_{-0.19}^{+0.20}$ & $27_{-4}^{+5}$ \\
\hline WISEA J030534.09-582635.5 & 8.0 & $13.60_{-0.30}^{+0.31}$ & $24.2_{-3.2}^{+3.7}$ & $667_{-83}^{+83}$ & $18.56_{-0.16}^{+0.17}$ & $47_{-7}^{+8}$ \\
\hline WISEA J032600.28+421056.8 & 8.5 & $13.74_{-0.30}^{+0.31}$ & $20.4_{-2.7}^{+3.1}$ & $626_{-82}^{+82}$ & $18.65_{-0.17}^{+0.19}$ & $45_{-7}^{+8}$ \\
\hline WISEA J032811.00-422321.6 & 8.5 & $13.70_{-0.31}^{+0.31}$ & $29.2_{-3.9}^{+4.5}$ & $638_{-85}^{+85}$ & $19.90_{-0.18}^{+0.19}$ & $82_{-13}^{+15}$ \\
\hline WISEA J034227.44-462252.0 & 7.5 & $13.33_{-0.31}^{+0.31}$ & $36.4_{-4.8}^{+5.5}$ & $761_{-85}^{+85}$ & $18.47_{-0.28}^{+0.32}$ & $51_{-10}^{+10}$ \\
\hline WISEA J035733.85+070557.4 & 6.5 & $13.11_{-0.30}^{+0.30}$ & $28.8_{-3.7}^{+4.3}$ & $866_{-82}^{+82}$ & $18.69_{-0.19}^{+0.20}$ & $62_{-10}^{+11}$ \\
\hline WISEA J040702.42+190945.8 & 6.5 & $13.15_{-0.30}^{+0.30}$ & $28.7_{-3.7}^{+4.3}$ & $841_{-82}^{+82}$ & $18.10_{-0.29}^{+0.34}$ & $46_{-9}^{+10}$ \\
\hline WISEA J042236.95-044203.5 & 8.0 & $13.52_{-0.31}^{+0.31}$ & $30.5_{-4.0}^{+4.6}$ & $692_{-84}^{+84}$ & $21.58_{-0.09}^{+0.09}$ & $194_{-27}^{+31}$ \\
\hline WISEA J050238.28+100750.0 & 9.0 & $14.05_{-0.31}^{+0.31}$ & $18.3_{-2.4}^{+2.8}$ & $553_{-83}^{+83}$ & $17.63_{-0.27}^{+0.31}$ & $25_{-5}^{+5}$ \\
\hline WISEU J050305.68-564834.0 & $\geqslant 11.0$ & $15.56_{-0.49}^{+0.51}$ & $12.4_{-2.6}^{+3.1}$ & $348_{-87}^{+89}$ & $20.70_{-0.07}^{+0.07}$ & $51_{-11}^{+13}$ \\
\hline WISEA J050615.56-514521.3 & 9.0 & $13.98_{-0.33}^{+0.34}$ & $28.3_{-4.1}^{+4.7}$ & $569_{-87}^{+88}$ & $18.40_{-0.28}^{+0.32}$ & $36_{-7}^{+8}$ \\
\hline WISEA J053512.01-773829.7 & 9.5 & $14.18_{-0.33}^{+0.33}$ & $21.9_{-3.1}^{+3.6}$ & $528_{-85}^{+85}$ & $17.88_{-0.26}^{+0.30}$ & $26_{-5}^{+5}$ \\
\hline WISEA J064503.72+524054.1 & 8.5 & $13.85_{-0.30}^{+0.31}$ & $18.1_{-2.4}^{+2.7}$ & $598_{-82}^{+82}$ & $20.13_{-0.05}^{+0.05}$ & $85_{-11}^{+13}$ \\
\hline WISEA J065113.90-835502.6 & 8.0 & $13.68_{-0.31}^{+0.31}$ & $23.5_{-3.1}^{+3.6}$ & $643_{-83}^{+83}$ & $17.84_{-0.19}^{+0.21}$ & $32_{-5}^{+6}$ \\
\hline WISEA J075438.20+090044.9 & 8.0 & $13.62_{-0.31}^{+0.31}$ & $26.6_{-3.5}^{+4.0}$ & $660_{-83}^{+83}$ & $18.30_{-0.33}^{+0.39}$ & $41_{-9}^{+9}$ \\
\hline WISEA J080622.22-082046.5 & 8.0 & $13.67_{-0.31}^{+0.31}$ & $22.3_{-2.9}^{+3.4}$ & $645_{-83}^{+83}$ & $21.14_{-0.06}^{+0.06}$ & $148_{-20}^{+23}$ \\
\hline WISEA J083011.95+283716.0 & $\geqslant 11.0$ & $16.19_{-0.55}^{+0.57}$ & $8.6_{-2.0}^{+2.5}$ & $303_{-85}^{+87}$ & $22.42_{-0.07}^{+0.07}$ & $84_{-19}^{+24}$ \\
\hline WISEA J083019.97-632305.4 & 8.0 & $13.59_{-0.31}^{+0.31}$ & $26.1_{-3.4}^{+4.0}$ & $668_{-83}^{+83}$ & $19.20_{-0.12}^{+0.12}$ & $63_{-9}^{+10}$ \\
\hline WISEA J084329.01+694709.8 & 6.0 & $13.01_{-0.30}^{+0.30}$ & $39.9_{-5.2}^{+5.9}$ & $928_{-83}^{+83}$ & $18.81_{-0.29}^{+0.34}$ & $69_{-13}^{+14}$ \\
\hline WISEA J101804.20-684254.0 & 8.5 & $13.71_{-0.31}^{+0.31}$ & $21.8_{-2.9}^{+3.3}$ & $633_{-83}^{+83}$ & $19.95_{-0.07}^{+0.07}$ & $84_{-11}^{+13}$ \\
\hline WISEA J104216.89-003935.9 & 7.5 & $13.37_{-0.30}^{+0.30}$ & $29.9_{-3.9}^{+4.5}$ & $744_{-83}^{+83}$ & $19.23_{-0.22}^{+0.24}$ & $70_{-12}^{+13}$ \\
\hline WISEA J105349.41-460241.2 & 8.5 & $13.69_{-0.30}^{+0.30}$ & $20.6_{-2.7}^{+3.1}$ & $639_{-82}^{+82}$ & $19.66_{-0.09}^{+0.09}$ & $74_{-10}^{+12}$ \\
\hline WISEA J105917.38+285729.3 & 7.5 & $13.45_{-0.30}^{+0.30}$ & $26.8_{-3.5}^{+4.0}$ & $713_{-83}^{+83}$ & $19.56_{-0.16}^{+0.17}$ & $79_{-12}^{+13}$ \\
\hline WISEA J110201.76+350335.4 & 9.0 & $14.04_{-0.31}^{+0.31}$ & $16.6_{-2.2}^{+2.5}$ & $556_{-82}^{+82}$ & $16.31_{-0.35}^{+0.41}$ & $13_{-3}^{+3}$ \\
\hline WISEA J112440.19+663052.0 & 8.0 & $13.62_{-0.30}^{+0.30}$ & $21.9_{-2.9}^{+3.3}$ & $660_{-82}^{+82}$ & $16.23_{-0.37}^{+0.45}$ & $16_{-4}^{+4}$ \\
\hline WISEA J114350.90+401333.9 & 8.5 & $13.70_{-0.31}^{+0.31}$ & $22.7_{-3.0}^{+3.4}$ & $636_{-83}^{+83}$ & $19.46_{-0.14}^{+0.15}$ & $67_{-10}^{+11}$ \\
\hline WISEA J114601.22+342458.8 & 7.0 & $13.20_{-0.30}^{+0.30}$ & $28.1_{-3.7}^{+4.2}$ & $815_{-82}^{+82}$ & $18.88_{-0.17}^{+0.18}$ & $65_{-10}^{+11}$ \\
\hline WISEA J115917.89+671704.2 & 9.0 & $14.08_{-0.32}^{+0.33}$ & $23.7_{-3.3}^{+3.8}$ & $547_{-85}^{+85}$ & $20.59_{-0.15}^{+0.16}$ & $95_{-15}^{+17}$ \\
\hline WISEA J125721.01+715349.3 & $\geqslant 11.0$ & $15.05_{-0.43}^{+0.45}$ & $16.6_{-3.1}^{+3.7}$ & $398_{-88}^{+90}$ & $21.05_{-0.12}^{+0.13}$ & $75_{-15}^{+17}$ \\
\hline CWISEP J135937.65-435226.9 & 9.5 & $14.19_{-0.33}^{+0.33}$ & $22.1_{-3.1}^{+3.6}$ & $525_{-85}^{+85}$ & $18.54_{-0.39}^{+0.47}$ & $35_{-8}^{+9}$ \\
\hline WISEA J143422.31-083934.2 & 9.0 & $13.93_{-0.32}^{+0.32}$ & $24.3_{-3.3}^{+3.8}$ & $580_{-84}^{+84}$ & $19.58_{-0.26}^{+0.29}$ & $64_{-12}^{+13}$ \\
\hline WISEA J151620.39+721745.4 & 9.0 & $14.15_{-0.33}^{+0.33}$ & $22.8_{-3.2}^{+3.7}$ & $532_{-85}^{+85}$ & $20.41_{-0.10}^{+0.10}$ & $85_{-13}^{+14}$ \\
\hline WISEA J152529.09+605356.5 & 9.0 & $14.03_{-0.32}^{+0.32}$ & $23.5_{-3.2}^{+3.7}$ & $559_{-84}^{+85}$ & $20.54_{-0.10}^{+0.10}$ & $95_{-14}^{+16}$ \\
\hline WISEAR J154025.77-113940.8 & 7.0 & $13.24_{-0.30}^{+0.30}$ & $34.5_{-4.5}^{+5.2}$ & $800_{-83}^{+84}$ & $20.64_{-0.18}^{+0.19}$ & $144_{-22}^{+25}$ \\
\hline WISEA J155349.96+693355.2 & 5.0 & $12.53_{-0.24}^{+0.25}$ & $38.6_{-4.2}^{+4.6}$ & - & $22.13_{-0.06}^{+0.06}$ & $395_{-44}^{+48}$ \\
\hline WISEA J160516.79+002139.0 & 8.5 & $13.72_{-0.31}^{+0.31}$ & $22.6_{-3.0}^{+3.4}$ & $633_{-83}^{+83}$ & $18.10_{-0.24}^{+0.26}$ & $36_{-6}^{+7}$ \\
\hline WISEA J162852.64+160421.0 & 8.5 & $13.76_{-0.31}^{+0.31}$ & $26.1_{-3.5}^{+4.0}$ & $621_{-84}^{+84}$ & $20.96_{-0.11}^{+0.11}$ & $131_{-19}^{+21}$ \\
\hline WISEA J163932.75+184049.4 & 9.5 & $14.25_{-0.31}^{+0.31}$ & $16.8_{-2.3}^{+2.6}$ & $514_{-83}^{+83}$ & $18.97_{-0.14}^{+0.15}$ & $42_{-6}^{+7}$ \\
\hline WISEA J171331.68+245000.9 & 7.5 & $13.43_{-0.31}^{+0.31}$ & $30.4_{-4.0}^{+4.6}$ & $721_{-83}^{+84}$ & $18.69_{-0.34}^{+0.40}$ & $54_{-11}^{+12}$ \\
\hline WISEA J172907.10-753017.0 & 7.0 & $13.18_{-0.30}^{+0.30}$ & $28.0_{-3.6}^{+4.2}$ & $826_{-82}^{+82}$ & $16.77_{-0.31}^{+0.36}$ & $25_{-5}^{+5}$ \\
\hline
\end{tabular}


Table 9

(Continued)

\begin{tabular}{|c|c|c|c|c|c|c|}
\hline Name & $\begin{array}{c}\text { SpT } \\
\text { (phototype) }\end{array}$ & $\begin{array}{l}M_{\mathrm{ch} 2} \\
(\mathrm{mag})\end{array}$ & $\begin{array}{l}\text { Distance } \\
\quad(\mathrm{pc})\end{array}$ & $\begin{array}{l}T_{\text {eff }} \\
(\mathrm{K})\end{array}$ & $\begin{array}{c}H_{\mathrm{ch} 2} \\
(\mathrm{mag})\end{array}$ & $\begin{array}{c}V_{\mathrm{tan}} \\
\left(\mathrm{km} \mathrm{s}^{-1}\right)\end{array}$ \\
\hline WISEA J175328.55-590447.6 & 9.0 & $14.04_{-0.31}^{+0.31}$ & $17.0_{-2.2}^{+2.6}$ & $555_{-82}^{+82}$ & $18.10_{-0.20}^{+0.22}$ & $31_{-5}^{+5}$ \\
\hline WISEA J181849.59-470146.9 & 8.5 & $13.87_{-0.30}^{+0.30}$ & $17.3_{-2.3}^{+2.6}$ & $593_{-82}^{+82}$ & $18.64_{-0.12}^{+0.13}$ & $43_{-6}^{+7}$ \\
\hline WISEA J193054.55-205949.4 & $\geqslant 11.0$ & $15.99_{-0.38}^{+0.39}$ & $7.1_{-1.2}^{+1.4}$ & $315_{-82}^{+83}$ & $21.10_{-0.06}^{+0.06}$ & $50_{-8}^{+10}$ \\
\hline WISEA J201833.67-141720.3 & 9.0 & $14.07_{-0.31}^{+0.31}$ & $15.3_{-2.0}^{+2.3}$ & $550_{-82}^{+82}$ & $16.47_{-0.34}^{+0.40}$ & $14_{-3}^{+3}$ \\
\hline WISEA J212510.91-730758.8 & 8.0 & $13.58_{-0.31}^{+0.31}$ & $24.9_{-3.3}^{+3.8}$ & $672_{-83}^{+83}$ & $19.33_{-0.15}^{+0.16}$ & $67_{-10}^{+11}$ \\
\hline WISEA J214025.23-332707.4 & 8.5 & $13.83_{-0.32}^{+0.32}$ & $28.7_{-4.0}^{+4.6}$ & $602_{-86}^{+86}$ & $20.22_{-0.22}^{+0.24}$ & $90_{-16}^{+17}$ \\
\hline WISEU J215018.46-752053.0 & 6.5 & $13.15_{-0.30}^{+0.30}$ & $31.4_{-4.1}^{+4.7}$ & $843_{-83}^{+83}$ & $20.56_{-0.09}^{+0.09}$ & $144_{-20}^{+22}$ \\
\hline WISEAR J220746.67-503631.7 & 7.5 & $13.41_{-0.31}^{+0.31}$ & $33.0_{-4.3}^{+5.0}$ & $729_{-84}^{+84}$ & $21.71_{-0.15}^{+0.16}$ & $216_{-32}^{+36}$ \\
\hline WISEA J221859.33+114644.4 & 7.5 & $13.35_{-0.30}^{+0.30}$ & $20.4_{-2.6}^{+3.0}$ & $753_{-82}^{+82}$ & $17.60_{-0.19}^{+0.21}$ & $34_{-5}^{+6}$ \\
\hline WISEA J221841.38+143003.4 & 7.5 & $13.36_{-0.30}^{+0.30}$ & $24.2_{-3.2}^{+3.6}$ & $748_{-82}^{+82}$ & $18.75_{-0.14}^{+0.15}$ & $57_{-8}^{+9}$ \\
\hline WISEA J233816.47-732929.7 & 9.0 & $13.97_{-0.31}^{+0.31}$ & $20.5_{-2.7}^{+3.1}$ & $571_{-83}^{+83}$ & $19.94_{-0.09}^{+0.09}$ & $74_{-10}^{+12}$ \\
\hline WISEA J235456.63-481440.1 & 9.5 & $14.17_{-0.32}^{+0.33}$ & $21.7_{-3.0}^{+3.5}$ & $529_{-84}^{+85}$ & $19.19_{-0.22}^{+0.25}$ & $48_{-8}^{+9}$ \\
\hline
\end{tabular}

Notes. Photometric spectral type values are defined such that $\mathrm{SpT}=6$ for $\mathrm{T} 6, \mathrm{SpT}=7$ for $\mathrm{T} 7, \ldots, \mathrm{SpT}=11$ for Y1. WISEA J053535.43-644518.5 is omitted from this table despite being motion confirmed, since its relatively blue ch1-ch2 $\approx 0$ color does not provide a well-constrained Spitzer-based phototype. WISEU J224547.11-433341.5 has been omitted because it lacks a ch1 magnitude and hence a Spitzer phototype.

${ }^{a}$ Bardalez Gagliuffi et al. (2020) present a trigonometric parallax for WISEA J083011.95+283716.0 yielding more reliable estimates for its phototype, $M_{\mathrm{ch} 2}$, distance, $T_{\text {eff }}$, and $V_{\text {tan }}$.

(This table is available in machine-readable form.)

\subsection{Absence of Discoveries as Cold as WISE 0855-0714}

Although WISEA $0830+2837$ may potentially be similar to WISE 0855-0714 (the coldest known brown dwarf) in terms of ch1-ch2 and F125W-ch2 colors, WISEA $0830+2837$ is $\sim 1.5$ mag more luminous at ch2 (Bardalez Gagliuffi et al. 2020) and thus presumably warmer. Therefore, recent Spitzer follow-up from Backyard Worlds and CatWISE appears not to have identified even one object as cold as or colder than WISE 0855-0714 ( $T_{\text {eff }} \approx 250 \mathrm{~K}$; Luhman $2014 \mathrm{~b}$ ). This may seem surprising given that motion surveys like Backyard Worlds and CatWISE are pushing much deeper than the relatively bright WISE 0855-0714 magnitude of W2 $\approx 14$ (see Figure 2). Wright et al. (2014) argued that a complete search of the WISE data to $\mathrm{W} 2=16$ should find between 3 (16th percentile) and 34 (84th percentile) additional objects ${ }^{25}$ comparable to WISE 0855-0714, with a median of 14 . The Backyard Worlds and CatWISE Spitzer target lists both have complex selection functions because they each amalgamate results from numerous contributing searches, but it is unlikely that either (or their union) is currently complete to W2 $=16$ (see esp. Section 3.3 of Eisenhardt et al. 2020 regarding CatWISE Preliminary incompleteness). Adjusting the Wright et al. (2014) prediction to the typical magnitude of our Backyard Worlds sample (W2 $\approx 15.7$; see Section 5) yields a corresponding 16th-84th percentile range of 1.6-22 WISE 0855-0714 analogs, with a median of 9 .

We find it conceivable that a small number of WISE 0855 -0714 analogs have been detected by WISE/NEOWISE but

\footnotetext{
25 These numbers are lower than those quoted in Wright et al. (2014) by 1 because the Wright et al. (2014) values include WISE 0855-0714 itself.
}

have so far not been pinpointed by either Backyard Worlds or CatWISE. For instance, the combined Backyard Worlds and CatWISE motion discovery lists total $\sim 4000$ objects, yet fewer than 300 of these were imaged with Spitzer. One cannot predict which WISE sources will turn out to be reddest in Spitzer based on faint W2 detections alone, so it is certainly possible that the parent Backyard Worlds plus CatWISE motion discovery samples contain new WISE 0855-0714 analogs not yet recognized as such owing to lack of Spitzer follow-up. This potential incompleteness affects both Backyard Worlds and CatWISE and could easily arise from, e.g., blending of an extremely cold brown dwarf with one or more background contaminants, yielding a spuriously blue $\mathrm{W} 1-\mathrm{W} 2$ color. Another selection bias specific to CatWISE is that very faint and fast-moving sources may not appear in the catalog at all, since CatWISE source detection is performed on static sky coadds spanning more than half a decade (Eisenhardt et al. 2020), significantly diluting the appearance of objects moving faster than $\sim 1^{\prime \prime} \mathrm{yr}^{-1}$.

To summarize, the absence of additional objects as cold as WISE 0855-0714 suggests that their true abundance is probably not on the higher side of imprecise estimates based purely on the nearby location of WISE $0855-0714$ (i.e., there likely are not of order 10 such objects with W2 $<15.7$ ). On the other hand, the absence of such discoveries in the Backyard Worlds and CatWISE Spitzer follow-up samples (zero identified vs. 1.6 expected at the distribution's 16 th percentile) does not definitively establish an underabundance relative to the Wright et al. (2014) forecast given the extremely low number statistics and possible incompleteness. An updated 20 pc census of brown dwarfs that incorporates recent Backyard 

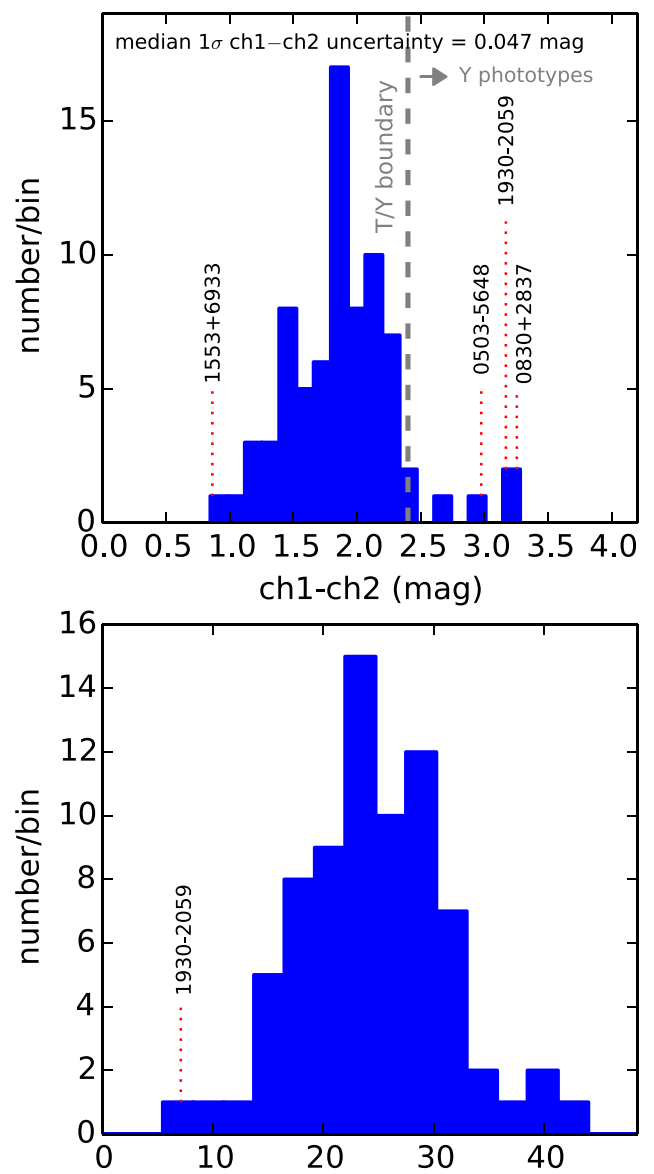

photometric distance estimate (pc)

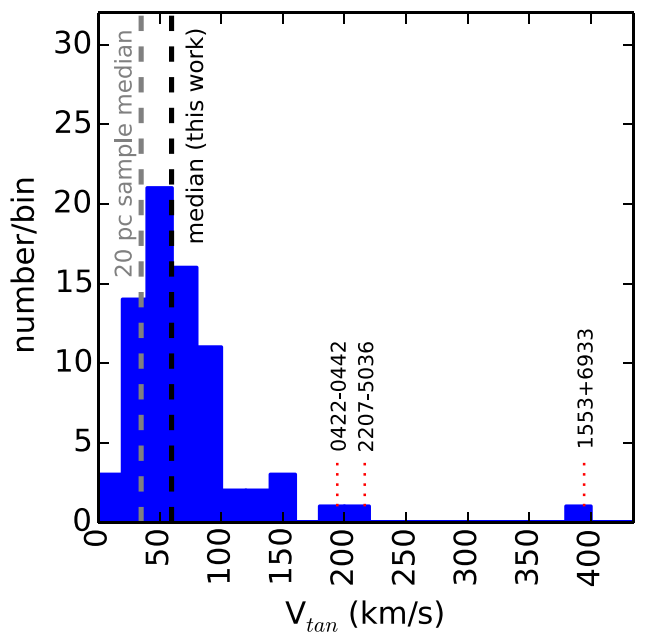

Figure 6. Distributions of Spitzer colors (top), photometric distance estimates (middle), and $V_{\tan }$ (bottom) from Table 9 for motion-confirmed targets whose spectral types could be estimated based on our Spitzer photometry. Short names of selected extreme objects are included as annotations.

Worlds and CatWISE discoveries can provide improved space density and mass function estimates, thereby enabling a more rigorous assessment of whether the close-by detection of WISE 0855-0714 is anomalous given its as-yet-unrivaled temperature. That analysis is beyond the scope of this work and will be presented in a dedicated follow-up paper (J. D. Kirkpatrick et al. 2020, in preparation).

\subsection{Objects with Largest Motions}

\subsubsection{Subdwarf Candidates}

Few mid-T or later subdwarfs are currently known, so identifying more such examples is a critical step toward developing an understanding of very cold substellar objects at low metallicity (e.g., Zhang et al. 2019). High tangential velocity is a potential indicator of a relatively old object with low metallicity possibly belonging to the Milky Way thick disk or halo. A threshold value for large $V_{\tan }$ sometimes employed in solar neighborhood studies is $100 \mathrm{~km} \mathrm{~s}^{-1}$ (e.g., Faherty et al. 2009). Ten objects in Table 9 have central $V_{\tan }$ estimates $>$ $100 \mathrm{~km} \mathrm{~s}^{-1}$. To highlight only the most extreme of our targets in terms of tangential velocity as potential subdwarfs, we examine the set of motion-confirmed targets with estimated $V_{\tan }>$ $150 \mathrm{~km} \mathrm{~s}^{-1}$ : WISEA 0422-0442 $\left(V_{\tan }=194_{-27}^{+31} \mathrm{~km} \mathrm{~s}^{-1}\right)$, WISEA $1553+6933\left(V_{\text {tan }}=395_{-44}^{+48} \mathrm{~km} \mathrm{~s}^{-1}\right)$, and WISEAR 2207-5036 $\left(V_{\tan }=216_{-32}^{+36} \mathrm{~km} \mathrm{~s}^{-1}\right)$. Mid- to late $\mathrm{T}$ subdwarfs may have unusually red $J-\operatorname{ch} 2$ colors relative to their ch1-ch2 colors (e.g., Mace et al. 2013), motivating a check of whether available NIR photometry indicates that our high- $V_{\tan }$ outliers are also color outliers. As shown in Figure 5, WISEAR 2207-5036 has a substantially redder $J-\operatorname{ch} 2$ color limit than would be expected for a normal brown dwarf with its ch1-ch2 $=1.66 \pm 0.06$ color, bolstering its subdwarf candidacy. On the other hand, WISEA $0422-0442$ has an NIR detection $(J=19.43 \pm 0.23)$, yielding a $J$ $-\operatorname{ch} 2=3.49 \pm 0.23$ color that is consistent with its T8 phototype.

WISEA $1553+6933$ stands apart from the rest of our brown dwarf candidates as a result of its exceedingly high tangential velocity estimate. WISEA $1553+6933$ is also our sample's fastest-moving source in terms of best-fit total linear motion $\left(\mu>2^{\prime \prime} \mathrm{yr}^{-1}\right)$. Despite not being especially faint by the standards of our sample (ch2 $\approx 15.5$ ), it was missed by prior searches owing to severe blending with a static background source during pre-hibernation WISE observations. Our OMM follow-up establishes a limit of $J>17.34$ for WISEA 1553 +6933 , corresponding to a $J-\mathrm{ch} 2>1.88$ color constraint. This color limit is not sufficiently stringent to rule out the possibility that WISEA $1553+6933$ is a normal mid-T brown dwarf. WISEA $1553+6933$ merits additional follow-up as a candidate mid- to late T-type subdwarf, including deeper $J$-band imaging.

\subsubsection{Reduced Proper Motion}

Reduced proper motion can be a valuable tool for identifying low-luminosity sources, such as Y dwarfs and subdwarfs, in the absence of trigonometric parallaxes. Since our sample lacks trigonometric parallaxes, we cannot directly compute absolute ch2 magnitudes to pinpoint our most intrinsically faint sources. Reduced proper motion replaces parallax in the absolute magnitude formula with total proper motion, on the premise that large apparent motion tends to indicate that a source is relatively nearby. Thus, we can single out objects of especially low luminosity within our sample (independent of absolute magnitude vs. color relations) based on their large reduced proper motions.

Figure 8 shows the reduced proper motions of our sample as a function of ch1-ch2 color. In this reduced proper-motion diagram, our sample's reddest objects (by ch1-ch2 color) are beginning to bridge a previously wide gap between the coldest known brown dwarf (WISE 0855-0714; Luhman 2014b) and 
$0503-5648 ; 2010.4$ to $2018.8 \quad 0830+2837 ; 2010.5$ to $2019.1 \quad 1257+7153 ; 2010.6$ to 2018.6
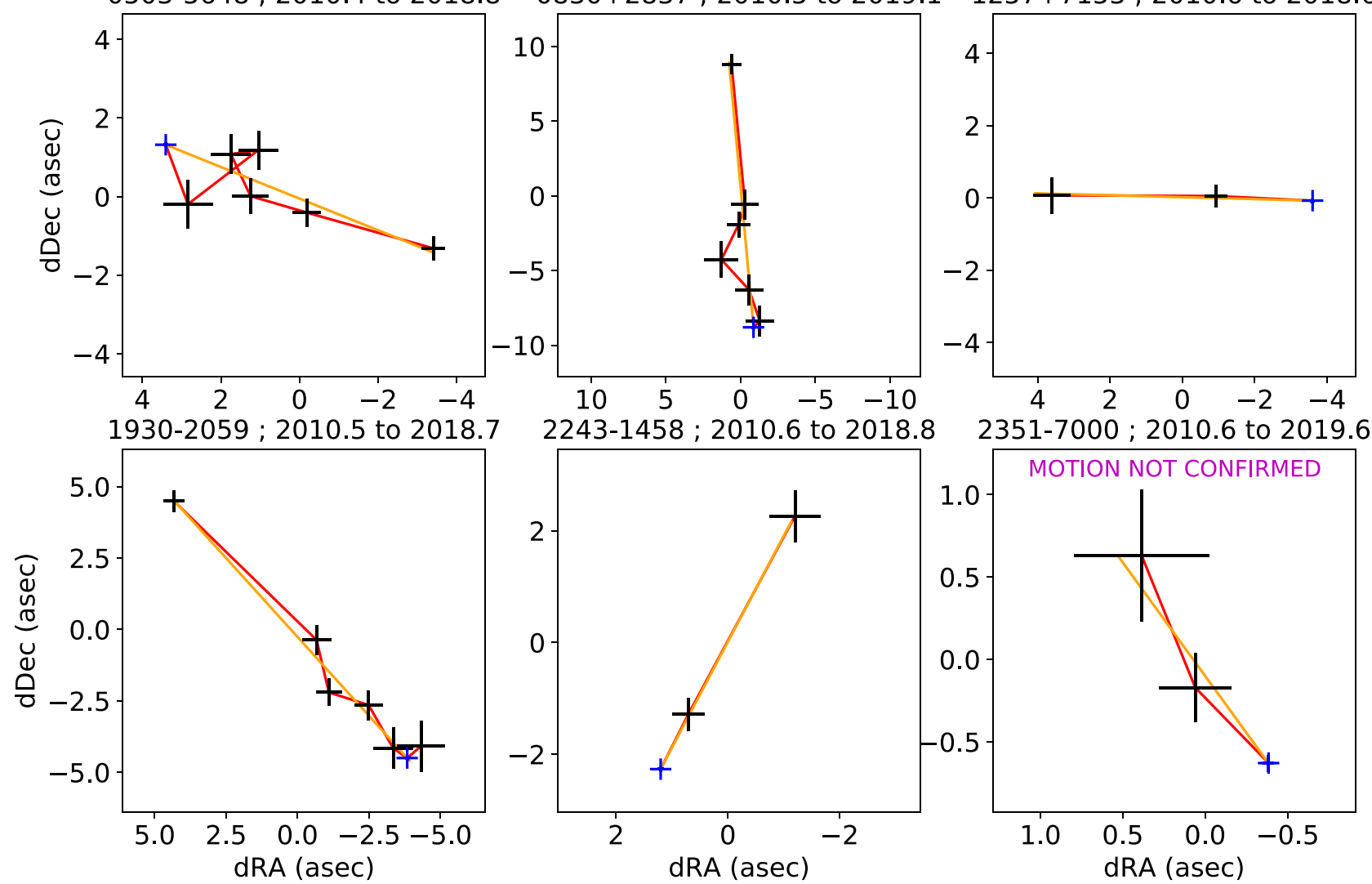

Figure 7. Combined WISE+Spitzer astrometry for our six targets with best-fit ch1-ch2 $>2.4$ mag, corresponding to phototypes $\geqslant Y 0$. Each panel's title lists the short object identifier and time period spanned by its astrometry. All but WISEA 2351-7000 are motion confirmed. The WISEA 2351-7000 trajectory appears plausibly linear, but its significance of motion falls just short of exceeding our $\chi_{\text {motion }}^{2}$ threshold for motion confirmation. dR.A. (dDecl.) is the positional offset along the R.A. (decl.) direction relative to the midway point between the minimum and maximum R.A. (decl.) for each object. dR.A. is in units of angular separation, rather than being a simple difference of R.A. coordinate values. Black plus signs represent the WISE W2 locations and their $\pm 1 \sigma$ uncertainties. Spitzer astrometry is represented by a blue plus sign of arbitrary size in each panel; the actual Spitzer uncertainties are much smaller than these symbols and would be difficult to perceive if plotted to scale. Red line segments follow the astrometric measurements in a time-ordered fashion. The best-fit linear motion solutions (Table 6) are shown as orange lines and cover the same time period spanned by the combined set of WISE and Spitzer detections. As illustrated by the relatively large size of the black plus signs, the time series of measured WISE positions can be quite noisy. For example, the loop-like phenomenon seen for WISEU 0503-5648 results from centroid measurement noise, not parallactic motion.

the rest of the Y dwarf population. WISEA $0830+2837$ in particular stands out as inhabiting this formerly empty region of parameter space and has the largest reduced proper motion of any member of our sample $\left(H_{\mathrm{ch} 2}=22.42 \pm 0.07 \mathrm{mag}\right)$. Note that WISEA $0830+2837$ is indeed alone in the gap between WISE 0855-0714 and other Y dwarfs. CWISEP 1446-2317 previously fell in a similar region of parameter space, but its ch1-ch2 color has recently been revised substantially blueward relative to that initially presented in Meisner et al. (2020). Still, the WISEA $0830+2837$ reduced proper motion is lower than that of WISE $0855-0714$ by more than a magnitude. As discussed in Bardalez Gagliuffi et al. (2020), WISEA 0830 +2837 may represent a heretofore missing link between the bulk of the as-yet-identified Y dwarfs and WISE 0855-0714. WISEA $1553+6933$ has the second-highest reduced proper motion among our sample, with $H_{\mathrm{ch} 2}=22.13 \pm 0.06$, while also being one of our bluest targets in ch1-ch2 color; we suspect that WISEA $1553+6933$ is a T-type subdwarf on account of its high kinematics (Section 11.5.1).

\subsubsection{Total Linear Motion}

Nine of our motion-confirmed discoveries have best-fit total linear motions larger than $1^{\prime \prime} \mathrm{yr}^{-1}$ : WISEU 0048+2508, WISEA
0422-0442, WISEA 0806-0820, WISEA 0830+2837, WISEA 1553+6933, WISEA 1628+1604, WISEA 1930-2059, WISEAR 2207-5036, and WISEA 2245-4333. Of these nine, two have best-fit total linear motions larger than $2^{\prime \prime} \mathrm{yr}^{-1}$ : WISEA $0830+2837$ and WISEA $1533+6933$. The suspected subdwarf WISEA $1553+6933$ has the largest total linear motion among

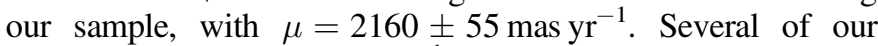
discoveries with $\mu_{\mathrm{tot}}>1^{\prime \prime} \mathrm{yr}^{-1}$ were missed by prior brown dwarf color selections owing to blending at early WISE epochs: CWISE 0002+6352, WISEU 0048+2508, WISEA 1553+6933, WISEA 1930-2059, and WISEU 2245-4333. By visually surveying for motion, Backyard Worlds citizen scientists were able to spot these previously overlooked members of the solar neighborhood.

\subsection{Candidate CPM Systems}

Five of our targets were considered potential CPM companions to earlier-type primaries upon being selected for Spitzer follow-up, and a sixth CPM candidate (CWISE 0002 +6352) has archival Spitzer data available. The comoving pair consisting of WISEU 2150-7520 and its L dwarf primary has already been discussed extensively in Faherty et al. (2020). WISEU $0505+3043$ was targeted as a possible comoving 


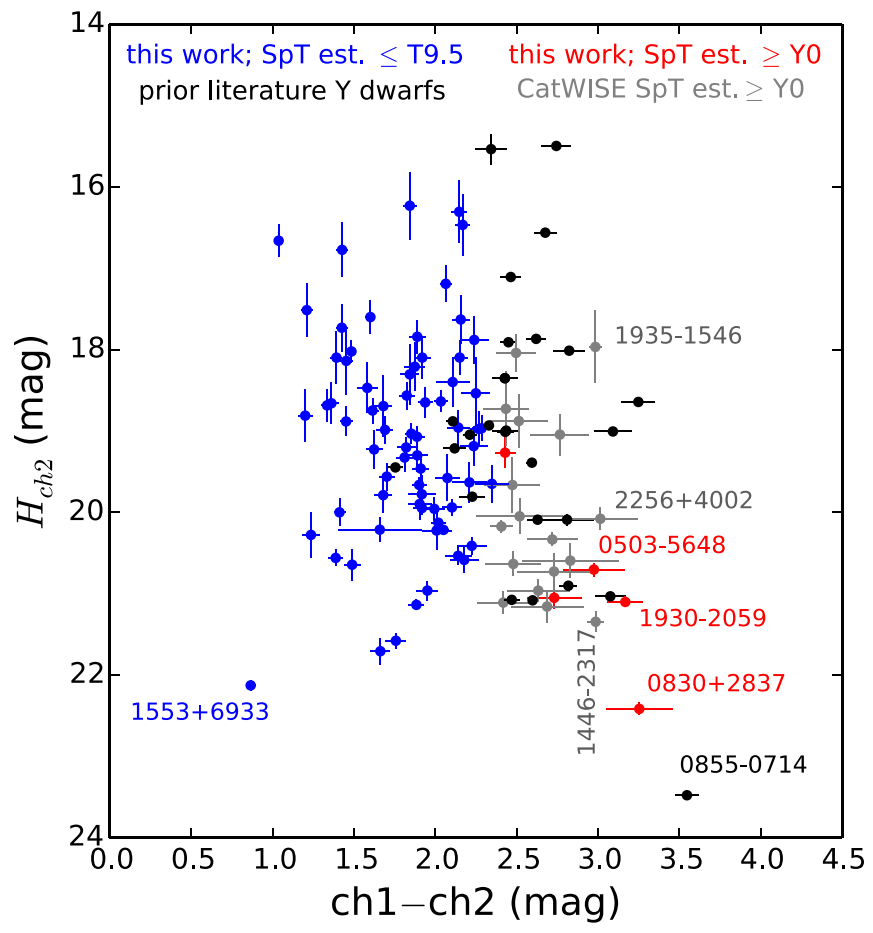

Figure 8. Reduced proper-motion diagram showing all Y dwarfs from the prior literature (black) and all motion-confirmed targets from this study with Spitzer phototypes available. Our targets with best-fit ch1-ch2 color most consistent with spectral type $\mathrm{Y}$ are shown in red, while all of our objects with earlier spectral type estimates are shown in blue. Recent CatWISE discoveries with Spitzer-based Y phototypes are also plotted in gray (Meisner et al. 2020). Our three discoveries with largest central $\operatorname{ch} 1-\operatorname{ch} 2$ colors are individually labeled (red annotations), as are the three reddest CatWISE discoveries (gray annotations; Marocco et al. 2019, 2020; Meisner et al. 2020).

companion to the extreme subdwarf LSPM J0505+3043 but turned out to be entirely spurious (see Table 1). The other remaining four CPM candidates are discussed in detail below.

\subsubsection{CWISE J000229.93+635217.0}

The motion of CWISE 0002+6352 appears strikingly similar to that of the $\sim 5$.5 distant DC white dwarf LSR J0002+6357 (Limoges et al. 2015), which has accurate parallax and propermotion measurements available from Gaia. Our WISE+Spitzer linear motion fitting gives $\mu_{\alpha}=802 \pm 49$ mas yr $^{-1}, \mu_{\delta}=44 \pm$ $47 \mathrm{mas} \mathrm{yr}^{-1}$ for CWISE $0002+6352$, versus $\mu_{\alpha}=918.9 \pm$ $0.1 \mathrm{mas} \mathrm{yr}^{-1}, \mu_{\delta}=108.3 \pm 0.1 \mathrm{mas} \mathrm{yr}^{-1}$ for LSR J0002+6357 (Gaia Collaboration et al. 2018). The CWISE 0002+6352 R.A. (decl.) motion component differs from that of LSR J0002+6357 by $2.4 \sigma(1.3 \sigma)$. Additionally, the Gaia parallax of LSR J0002 +6357 (38.1 mas, corresponding to $26.3 \mathrm{pc}$ ) places it within the $1 \sigma$ photometric distance interval of CWISE 0002+6352 $\left(28.6_{-5.1}^{+5.6}\right.$ pc; Table 9). Restricting to this distance range, only 12 sources in the entire Gaia DR2 catalog have both proper-motion components consistent with those of CWISE $0002+6352$ to within $3 \sigma$. Therefore, the probability of having one such Gaia source land at least as close on the sky as LSR J0002+6357 does to CWISE $0002+6352$ by random chance is $7.7 \times 10^{-6}$.

While this false-alarm probability suggests a good likelihood that CWISE $0002+6352$ is a bona fide companion to LSR $\mathrm{J} 0002+6357$, the moderate motion discrepancies remain

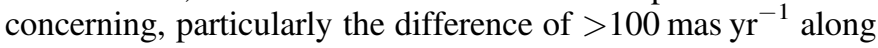
the R.A. direction. Significant blending of the brown dwarf's WISE counterpart at essentially all epochs in this highly crowded field (see Figure 9) means that our WISE+Spitzer motion may carry systematic uncertainties larger than those we quote based on statistics alone. In addition to a spectroscopic confirmation of CWISE $0002+6352$ to corroborate (or discredit) its photometric distance estimate, a future NIR astrometric data point free of blending would help provide a more conclusive determination as to whether CWISE 0002 +6352 is indeed physically associated with LSR J0002+6357. Based on its Spitzer photometry, we predict $J \approx 18.8$ for CWISE $0002+6352$. CWISE $0002+6352$ is not detected by Pan-STARRS (Chambers et al. 2016).

If CWISE $0002+6352$ and LSR J0002+6357 are physically associated, their angular separation of 330." $9 \pm 0 . " 4$ would translate to a projected physical separation of $8691 \pm 20$ au. This would be the second-largest projected physical separation of any known white dwarf plus mid- to late $\mathrm{T}$ dwarf system, following only LSPM 1459+0857AB (16,500-26,500 au; Day-Jones et al. 2011). LSR J0002+6357 has serendipitous Spitzer observations from the GLIMPSE360 program, with $\operatorname{ch} 1=15.05 \pm 0.05$ and $\operatorname{ch} 2=14.91 \pm 0.08$, though it appears slightly blended with a neighboring source in that archival imaging (see Figure 9).

\subsubsection{WISEU J001908.31-094323.3}

Based on visual inspection, we recognized WISEU 0019 -0943 to share a very similar motion with the nearby M dwarf LP 704-85. Our WISEU 0019-0943 linear motion agrees with the Gaia DR2 proper motion of LP 704-85 to within $1 \sigma$ in terms of both $\mu_{\alpha}$ and $\mu_{\delta}$. WISEU 0019-0943 is 19 !" $1 \pm$ 0 ". 4 distant from LP 704-85, comparing the Gaia epoch 2015.5 position of LP $704-85$ to that predicted at the same epoch by our linear motion model for WISEU 0019-0943. Using the full-sky Gaia DR2 catalog, we find that there are only 2601 Gaia sources that have both $\mu_{\alpha}$ and $\mu_{\delta}$ within $1 \sigma$ of the values listed for WISEU 0019-0943 in Table 6. This yields a chance alignment probability of $5.6 \times 10^{-6}$.

However, our Spitzer-based photometric distance estimate for WISEU 0019-0943 is in some tension with the Gaia DR2 parallax for LP 704-85. The WISEU 0019-0943 photometric distance estimate $\left(d=31.1_{-4.0}^{+4.6} \mathrm{pc}\right)$ is $3 \sigma$ discrepant from the LP 704-85 distance according to Gaia DR2 $(d=44.9 \pm 0.2$ pc). We note that this discrepancy could be eliminated if WISEU 0019-0943 were itself a pair of T7 brown dwarfs rather than a single $\mathrm{T} 7$ brown dwarf, which would then place its central distance estimate at 44.0 pc. Archival VHS images do show a pair of sources near the WISEU 0019-0943 location, separated by 3". 4. However, the WISEU 0019-0943 Spitzer ch2 counterpart looks pointlike, whereas a $\sim 3^{\prime \prime}$ separation pair of sources with similar ch2 apparent brightnesses should have yielded a significantly extended profile given that the ch2 PRF FWHM is $\sim 2^{\prime \prime}$. We therefore believe that one of the nearby VHS NIR detections (the fainter source in $J$ band) is an unrelated background object. We consider our current data to be inconclusive regarding whether WISEU 0019 -0943 is indeed physically associated with LP 704-85. If physically associated, the projected separation would be $857 \pm 16$ au. LP 704-85 has no published spectrum, but its $J_{2 \text { MASS }}$ apparent magnitude and Gaia parallax combine to give an absolute $J_{2 \text { MASS }}$ magnitude of 8.9 , consistent with spectral type $\sim$ M4 (Hawley et al. 2002). 
Spitzer; 2010.1

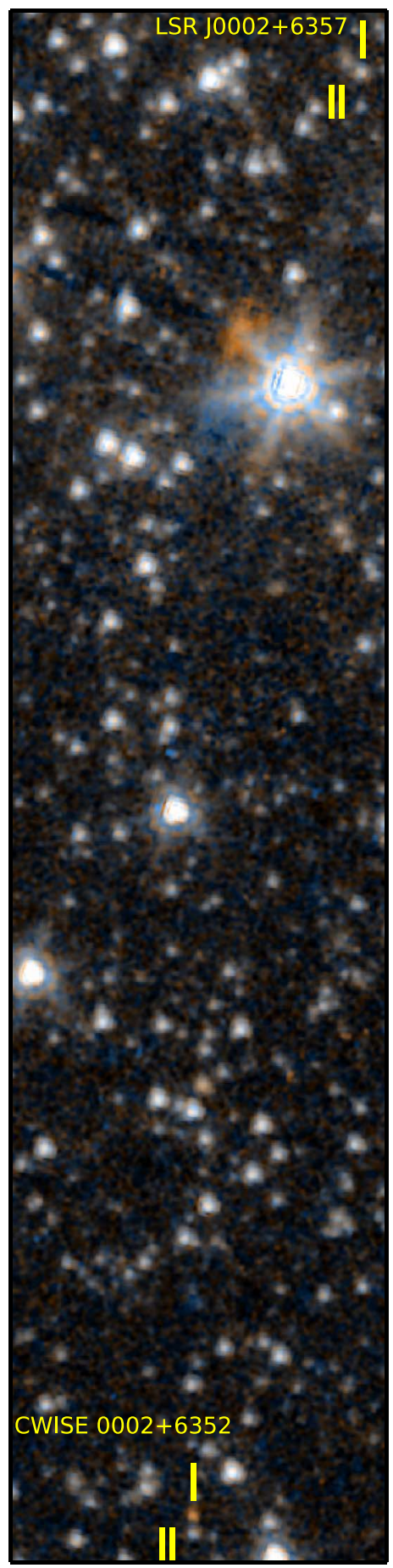

WISE; 2014.1-2015.6

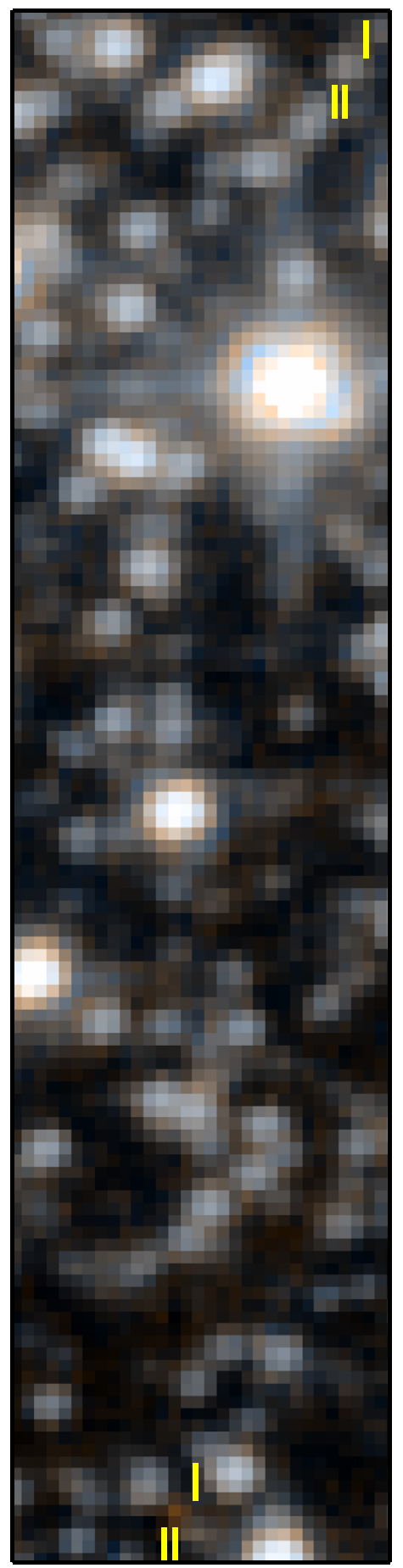

WISE; 2016.0-2017.6

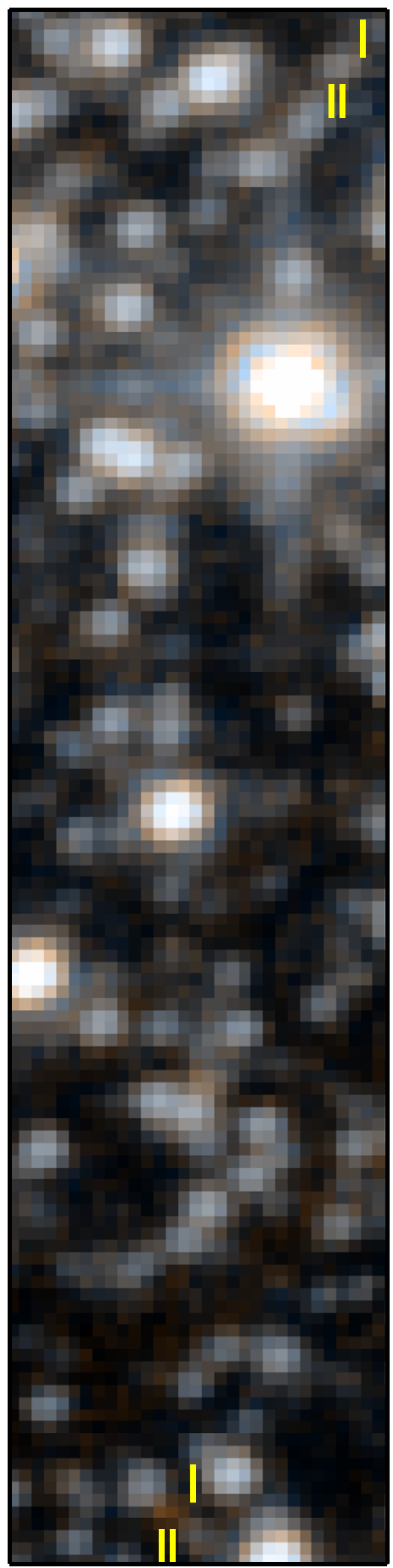

Figure 9. Time series of WISE and Spitzer images illustrating the similar proper motions of our late T discovery CWISE $0002+6352$ (bottom of each cutout) and the DC white dwarf LSR J0002+6357 (upper right of each cutout). Each panel is a two-band color-composite rendering. In each case W1 (ch1) is represented by the blue color channel and W2 (ch2) is represented by the red color channel. The Spitzer color composite at left is built from archival GLIMPSE360 imaging. WISE images (middle and right panels) are co-adds spanning different portions of the post-reactivation time period. CWISE $0002+6352$ appears distinctively orange in comparison to the relatively white/blue background source population that has $\mathrm{W} 1-\mathrm{W} 2 \approx \mathrm{ch} 1-\mathrm{ch} 2 \approx 0$. East is left and north is up. Yellow vertical lines track the west-to-east motion of this pair over the $\sim 2010-2018$ time period. Each postage stamp is $1 ! 6 \times 6 ! 2$ in angular extent. Follow-up NIR astrometry would help to conclusively determine whether CWISE $0002+6352$ and LSR J0002+6357 are indeed physically associated by refining the former's measured proper motion.

\subsubsection{WISEU J005559.88+594745.0}

Based on visual inspection, we recognized WISEU 0055 +5947 to share a very similar motion with the nearby DC white dwarf LSPM J0055+5948 (see Figure 10; Limoges et al. 2013). Our WISEU $0055+5947$ linear motion agrees with the
Gaia DR2 proper motion of LSPM J0055+5948 to within $2 \sigma$ in terms of both $\mu_{\alpha}$ and $\mu_{\delta}$. WISEU $0055+5947$ is 17 !" $6 \pm 0$ !. 1 distant from LSPM J0055+5948, comparing the Gaia epoch 2015.5 position of LSPM J0055+5948 to that predicted at the same epoch by our linear motion model for WISEU 

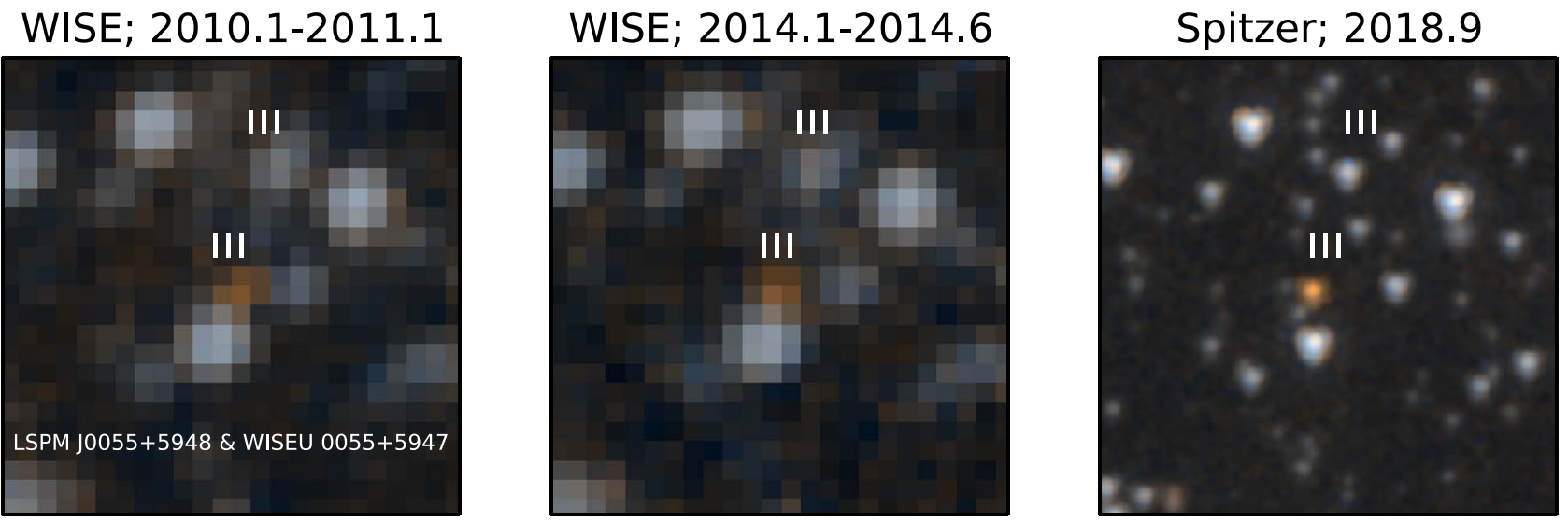

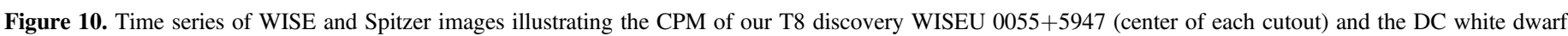

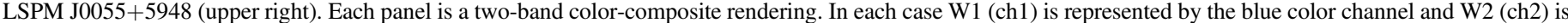

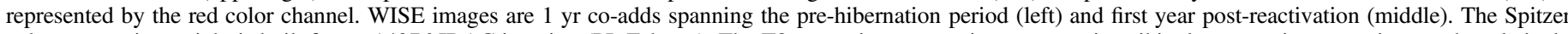

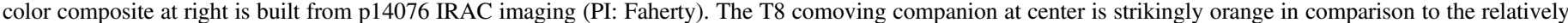

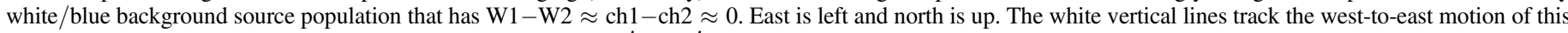
pair over the $\sim 2010-2019$ time period. Each postage stamp is $1 ! 1 \times 1 ! 1$ in angular extent.

0055+5947. Using the full-sky Gaia DR2 catalog, we find that there are only 351 Gaia sources that have both $\mu_{\alpha}$ and $\mu_{\delta}$ within $2 \sigma$ of the values listed for WISEU $0055+5947$ in Table 6 . This yields a chance alignment probability of just $6.4 \times 10^{-7}$.

The WISEU $0055+5947$ photometric distance estimate provides further evidence for physical association with LSPM J0055+5948. The white dwarf has a Gaia parallax of $43.78 \pm 0.07$ mas, corresponding to a distance of $22.8 \mathrm{pc}$. This agrees with our Spitzer-based photometric distance estimate for WISEU $0055+5947\left(d=22.2_{-2.9}^{+3.3} \mathrm{pc}\right.$; Table 9) to within $1 \sigma$. Adding further corroboration, our Keck/NIRES spectral type of T8 for the secondary (Section 8) agrees with our photometric estimate of T7 within the expected phototyping uncertainty (Section 11.1). The chance alignment probability drops further to $4.9 \times 10^{-8}$ when accounting for the consistency of the brown dwarf distance estimate with the white dwarf parallax in combination with the similarity of their proper motions. The projected physical separation between the brown dwarf and white dwarf is $402 \pm 3$ au using the white dwarf's Gaia parallax to convert from arcseconds of angular separation to projected au. We conclude that WISEU 0055 +5947 is indeed a comoving companion of LSPM J0055 +5948 . This makes WISEU $0055+5947$ an interesting target for further benchmarking studies given the potential for determining the system's age using that of the white dwarf primary.

LSPM J0055+5948 was identified as a DC white dwarf by Limoges et al. (2015) and is also in the Gentile Fusillo et al. (2019) Gaia DR2 white dwarf catalog. According to Gentile Fusillo et al. (2019), LSPM J0055 +5948 has $T_{\text {eff }}=4623 \pm$ $21 \mathrm{~K}, \log (g)=7.82 \pm 0.02$, and $M=0.474 \pm 0.010 M_{\odot}$ when modeled assuming a pure $\mathrm{H}$ atmosphere (Gentile Fusillo et al. 2019), as suggested by Limoges et al. (2013). To confirm these parameters, we also obtained the Pan-STARRS griz (Chambers et al. 2016), 2MASS $J H K_{s}$, and AllWISE W1,W2 photometry of LSPM J0055+5948 and determined $T_{\text {eff }}$ and $\log (g)$ using the photometric spectral energy distribution (SED) and Gaia DR2 parallax. For a DA composition and fitting to the Fontaine et al. (2001) cooling models, we obtain $T_{\text {eff }}=4734 \pm 81 \mathrm{~K}$ and $\log$ $(g)=7.87 \pm 0.07$, consistent within the uncertainties with the Gentile Fusillo et al. (2019) values.
Using the Gentile Fusillo et al. (2019) parameters and the cooling models from Fontaine et al. (2001) for DA white dwarfs, we determine a cooling time of $5.01_{-0.23}^{+0.24}$ Gyr. We then use the semiempirical initial mass-final mass relationship for white dwarfs from Cummings et al. (2018) to determine the initial mass of the white dwarf progenitor to be $1.38_{-0.21}^{+0.47} M_{\odot}$, a likely solar-type star. Combining the progenitor mass with the MIST isochrones (Paxton et al. 2011; Dotter 2016), we determine the total age of the system to be $10 \pm 3$ Gyr. The age we determine is consistent if we use the SED determined $T_{\text {eff }}$ and $\log (g)$.

It should be noted that while we have assumed that this DC white dwarf is likely to have a hydrogen-rich atmosphere, the Gentile Fusillo et al. (2019) modeling gives $T_{\text {eff }}=4654 \pm 17$ $\mathrm{K}, \log (g)=7.84 \pm 0.02$, and $M=0.473 \pm 0.010 M_{\odot}$ when modeled assuming a pure $\mathrm{He}$ atmosphere, which is also consistent with the values we have determined for the white dwarf, meaning that even if LSPM J0055+5948 is a helium atmosphere white dwarf, our estimate of the system age will not change. LSPM J0055 +5948 has ch1 $=14.91 \pm 0.01$ and ch2 $=14.90 \pm 0.02$ based on our Spitzer follow-up imaging.

Combining our total system age constraint and $T_{\text {eff }}$ estimate from Table 9 yields a mass range of $56 \pm 9 M_{\text {Jup }}$ for WISEU $0055+5947$ based on the Saumon \& Marley (2008) model grids.

The WISEU $0055+5947$ plus LSPM J0055+5948 pair is currently only the fourth such wide system to be discovered after LSPM 1459+0857AB (Day-Jones et al. 2011), WD 0806661 AB (Luhman et al. 2011), and COCONUTS-1AB (Zhang et al. 2020). The projected separation of $\sim 400$ au for WISEU $0055+5947$ is closer than the other three comoving systems, all of which have projected separations of at least $1000 \mathrm{au}$ (COCONUTS-1AB: $1280 \mathrm{au;}$ WD 0806-661AB: $2500 \mathrm{au}$; LSPM 1459+0857AB: 16500-26500 au). All four systems have $\mathrm{T}$ dwarf companions or later: both COCONUTS-1B and LSPM $1459+0857 \mathrm{~B}$ are T4/5 dwarfs, and WD $0806-661 \mathrm{~B}$ is a probable $\mathrm{Y}$ dwarf and is almost certainly of planetary mass (Luhman et al. 2014). All systems are old, with ages of at least 2 Gyr, and WISEU $0055+5947$ is probably the oldest, based on its low effective temperature and age estimate. The wide separation of the brown and white dwarfs means that the brown 
dwarf is unlikely to have been affected by the evolution of the white dwarf progenitor and also that the white dwarf evolution is unlikely to have been truncated during a common envelope phase. This is supported by the fact that none of the white dwarfs in these systems are of low enough mass to have formed via binary evolution (e.g., Marsh 1995). The fact that the components of these binaries are comoving but have not affected each other's evolution makes them ideal benchmark systems for determining spectroscopic parameters of old brown dwarfs.

\subsubsection{WISEA J075438.20+090044.9}

Based on WISE/NEOWISE imaging alone, the WISEA 0754 +0900 motion appears visually similar to that of the $780^{\prime \prime}$ distant M6 dwarf LP 483-66. The WISE-based proper motion of WISEA $0754+0900 \quad\left(\mu_{\alpha}=267 \pm 72\right.$ mas yr $^{-1}, \quad \mu_{\delta}=-179 \pm$ 90 mas yr $^{-1}$ according to the CatWISE 2020 catalog) is consistent with the Gaia DR2 proper motion of LP 483-66 $\left(\mu_{\alpha}=414\right.$ mas yr $^{-1}, \mu_{\delta}=-233$ mas yr $\left.^{-1}\right)$ to within $\sim 2 \sigma$ along both directions. Our photometric distance estimate for WISEA $0754+0900\left(26.6_{-3.5}^{+4.0} \mathrm{pc}\right.$; Table 9) is also consistent with the LP 483-66 parallax from Gaia (33.2 mas, corresponding to a distance of $30.1 \mathrm{pc}$ ). However, our WISE+Spitzer motion $\left(\mu_{\alpha}=166 \pm 52 \mathrm{mas} \mathrm{yr}^{-1}, \mu_{\delta}=-280 \pm 55 \mathrm{mas} \mathrm{yr}^{-1}\right.$; Table 6) differs substantially from the LP 483-66 Gaia proper motion, with a discrepancy of nearly $5 \sigma$ along the R.A. direction. No anomalies are evident in our WISEA 0754+0900 ch2 imaging/ astrometry, so we view our WISE+Spitzer linear motion as superior to estimates based solely on WISE. As a result, we consider it unlikely that WISEA 0754+0900 and LP 483-66 are associated given the available evidence.

\subsection{Notes on Individual Objects}

\subsubsection{WISEA J035410.03-572104.0}

WISEA $0354-5721$ has $\chi_{\text {motion }}^{2}=16.7$ and therefore is not motion confirmed by our WISE+Spitzer astrometry analysis. Nevertheless, this source appears to have a very faint/red counterpart moving southward when comparing $z$-band images from DECaLS DR8 (Dey et al. 2019) with those from Dark Energy Survey DR1 (Abbott et al. 2018). The $z$-band counterpart has $z=22.52 \pm 0.15(\mathrm{AB})$ according to DECaLS DR8. Assuming that this $z$-band source is indeed linked to our WISE/Spitzer brown dwarf candidate, then the object would be most consistent with a $\sim 77.5$ phototype and a distance of $\sim 33$ pc.

\subsubsection{WISEA J053535.43-644518.5}

WISEA 0535-6445 is unusual among our sample in that its ch1-ch2 $\approx 0$ color is not indicative of a mid-T or later brown dwarf, but rather is consistent with a broad range of earlier spectral types (Patten et al. 2006). Little archival photometry is available for this object, which lacks counterparts in Gaia and 2MASS while falling outside of the VHS footprint. The 2MASS nondetection implies a $J-\mathrm{W} 2 \gtrsim 1.9$ color limit. WISEA 0535 -6445 has $\mathrm{W} 1-\mathrm{W} 2=0.17 \pm 0.04$ according to the CatWISE Preliminary catalog, with slightly redder WISE colors of W1 $-\mathrm{W} 2=0.23 \pm 0.05$ and $\mathrm{W} 1-\mathrm{W} 2=0.29 \pm 0.02$ according to the AllWISE and unWISE Catalogs, respectively. The NOAO Source Catalog (Nidever et al. 2018) reports a DECam counterpart with $\mathrm{AB}$ magnitudes of $i=21.87 \pm 0.07$ and $z=20.36 \pm 0.06$. The $J-\mathrm{W} 2$ lower bound, $\mathrm{W} 1-\mathrm{W} 2$ color, and $i-z$ color indicate an early $\mathrm{L}$ type, and $i-\mathrm{W} 2=6$. $61 \pm 0.08$ suggests a spectral type of $\sim$ L3. The WISEA 0535 -6445 photometry is not consistent with an M subdwarf or $\mathrm{L}$ subdwarf scenario. If WISEA 0535-6445 is an L3 dwarf, then its photometric distance estimate would be $\sim 84$ pc, corresponding to a tangential velocity of $\sim 112 \mathrm{~km} \mathrm{~s}^{-1}$.

\subsubsection{CWISEP J135937.65-435226.9}

CWISEP 1359-4352 was inadvertently targeted as part of p14299 despite the fact that CatWISE p14034 had previously observed it (Meisner et al. 2020). Our p14299 observations of this object are deeper than those from p14034 and so provide a higher-S/N color estimate of ch1-ch2 = 2.25 $\pm 0.09 \mathrm{mag}$, corresponding to a best-fit spectral type estimate of T9.5. The $1 \sigma$ ch $1-\operatorname{ch} 2$ color intervals from p14034 and p14299 overlap for this source, although the central color value from Meisner et al. (2020) had scattered sufficiently redward to yield a phototype of Y0 rather than T9.5 as we have obtained here.

\section{Conclusion}

We have presented critical Spitzer photometric follow-up of 95 brown dwarf candidates discovered by the Backyard Worlds citizen science project. Our Spitzer astrometry allows us to verify the motions of most of these objects, certifying that these are new members of the solar neighborhood. Additionally, Keck/NIRES and Magellan/FIRE spectroscopy of 10 candidates confirms in all cases that the targets are brown dwarfs. Our Spitzer imaging also yields photometric spectral types and distances; these estimates are crucial for pinpointing the superlative objects among our sample, separating out the strong Y dwarf candidates from the late T dwarfs.

Among our most exciting discoveries are new candidate members of the $10 \mathrm{pc}$ sample, two objects moving faster than $2^{\prime \prime} \mathrm{yr}^{-1}$, three T-type subdwarf candidates, five $\mathrm{Y}$ dwarf candidates, and a new T8 plus white dwarf comoving system. Our Y dwarf candidates begin bridging the gap between the bulk of the Y dwarf population and the coldest known brown dwarf, making them potential targets for JWST spectroscopy. Backyard Worlds is actively pursuing additional ground-based follow-up of the discoveries presented in this study, especially spectroscopy where feasible. While this work's new brown dwarf candidates already demonstrate the power of citizen science for mapping the solar neighborhood, these objects make up only a small fraction of Backyard Worlds moving object discoveries to date. As NEOWISE continues scanning the sky, Backyard Worlds will endeavor to search all of its newly delivered data for yet more cold and close neighbors to the Sun.

We thank the anonymous referee for valuable comments. The Backyard Worlds: Planet 9 team would like to thank the many Zooniverse volunteers who have participated in this project, from providing feedback during the beta review stage to classifying flipbooks to contributing to the discussions on TALK. We would also like to thank the Zooniverse web development team for their work creating and maintaining the Zooniverse platform and the Project Builder tools. This research was supported by NASA grant 2017-ADAP17-0067. S.L.C. acknowledges the support of an STFC Ernest Rutherford Fellowship. Support for this work was provided by NASA through the NASA Hubble Fellowship grant HST-HF2-51447.001-A awarded by the Space Telescope Science 
Institute, which is operated by the Association of Universities for Research in Astronomy, Inc., for NASA, under contract NAS526555. This publication makes use of data products from the Wide-field Infrared Survey Explorer, which is a joint project of the University of California, Los Angeles, and the Jet Propulsion Laboratory/California Institute of Technology, funded by the National Aeronautics and Space Administration. This research has made use of the NASA/IPAC Infrared Science Archive, which is funded by the National Aeronautics and Space Administration and operated by the California Institute of Technology. This research has made use of the VizieR catalog access tool, CDS, Strasbourg, France (DOI :10.26093/cds/vizier). The original description of the VizieR service was published in Ochsenbein et al. (2000). This work has made use of the white dwarf cooling models hosted by Pierre Bergeron at http://www.astro.umontreal.ca/ -bergeron/ CoolingModels.

Facilities: Spitzer(IRAC), HST(WFC3), WISE/NEOWISE, UKIRT(WFCAM), VISTA(VIRCAM), IRSA, 2MASS, Gaia.

Software: MOPEX (Makovoz \& Khan 2005; Makovoz \& Marleau 2005), WiseView (Caselden et al. 2018), astrometry. net (Lang et al. 2010).

\section{ORCID iDs}

Aaron M. Meisner (10 https://orcid.org/0000-0002-1125-7384 Jacqueline K. Faherty (1) https://orcid.org/0000-0001-6251-0573 J. Davy Kirkpatrick (1) https://orcid.org/0000-0003-4269-260X Adam C. Schneider (ib https://orcid.org/0000-0002-6294-5937 Dan Caselden (1) https://orcid.org/0000-0001-7896-5791 Jonathan Gagné (1) https://orcid.org/0000-0002-2592-9612 Marc J. Kuchner (1) https://orcid.org/0000-0002-2387-5489 Adam J. Burgasser (1) https://orcid.org/0000-0002-6523-9536 Sarah L. Casewell (1) https://orcid.org/0000-0003-2478-0120 John H. Debes (1) https://orcid.org/0000-0002-1783-8817 Étienne Artigau (1) https://orcid.org/0000-0003-3506-5667 Daniella C. Bardalez Gagliuffi 1 h https://orcid.org/0000-00018170-7072

Sarah E. Logsdon (1) https://orcid.org/0000-0002-9632-9382

Rocio Kiman (10 https://orcid.org/0000-0003-2102-3159

Katelyn Allers (1) https://orcid.org/0000-0003-0580-7244 Chih-chun Hsu (i) https://orcid.org/0000-0002-5370-7494 John P. Wisniewski (1) https://orcid.org/0000-0001-9209-1808 Guillaume Colin (1) https://orcid.org/0000-0002-7630-1243 Hugo A. Durantini Luca (ํ) https://orcid.org/0000-00024143-2550

Sam Goodman (주 https://orcid.org/0000-0003-2236-2320 Léopold Gramaize (1) https://orcid.org/0000-0002-8960-4964 Leslie K. Hamlet (10) https://orcid.org/0000-0002-7389-2092 Ken Hinckley $\mathbb{1}$ (1) https://orcid.org/0000-0002-4733-4927 Frank Kiwy (i) https://orcid.org/0000-0001-8662-1622 Austin Rothermich (1) https://orcid.org/0000-0003-4083-9962 Arttu Sainio (1) https://orcid.org/0000-0003-4864-5484 Jörg Schümann (1) https://orcid.org/0000-0002-7587-7195 Christopher Tanner (1) https://orcid.org/0000-0002-9807-5435 Melina Thévenot (i) https://orcid.org/0000-0001-5284-9231

\section{References}

Abbott, T. M. C., Abdalla, F. B., Allam, S., et al. 2018, ApJS, 239, 18 Artigau, E., Doyon, R., Vallee, P., Riopel, M., \& Nadeau, D. 2004, Proc. SPIE, 5492, 1479

Bardalez Gagliuffi, D. C., Faherty, J. K., Schneider, A. C., et al. 2020, ApJ, 895,145

Batygin, K., \& Brown, M. E. 2016, AJ, 151, 22
Beichman, C., Gelino, C. R., Kirkpatrick, J. D., et al. 2013, ApJ, 764, 101 Bochanski, J. J., Hennawi, J. F., Simcoe, R. A., et al. 2009, PASP, 121, 1409 Burgasser, A. J., McElwain, M. W., Kirkpatrick, J. D., et al. 2004, AJ, 127, 2856

Caselden, D., Westin, P., III, Meisner, A., Kuchner, M., \& Colin, G. 2018, WiseView: Visualizing Motion and Variability of Faint WISE Sources, Astrophysics Source Code Library, ascl:1806.004

Chambers, K. C., Magnier, E. A., Metcalfe, N., et al. 2016, arXiv:1612.05560

Churchwell, E., Babler, B. L., Meade, M. R., et al. 2009, PASP, 121, 213

Cross, N. J. G., Collins, R. S., Mann, R. G., et al. 2012, A\&A, 548, A119

Cummings, J. D., Kalirai, J. S., Tremblay, P. E., Ramirez-Ruiz, E., \& Choi, J. 2018, ApJ, 866, 21

Cushing, M. C., Kirkpatrick, J. D., Gelino, C. R., et al. 2011, ApJ, 743, 50

Cushing, M. C., Vacca, W. D., \& Rayner, J. T. 2004, PASP, 116, 362

Cutri, R. M., Mainzer, A., Conrow, T., et al. 2015, Explanatory Supplement to the NEOWISE Data Release Products, Tech. Rep. 1

Cutri, R. M., Wright, E. L., Conrow, T., et al. 2012, Explanatory Supplement to the WISE All-Sky Data Release Products, Tech. Rep. 1

Cutri, R. M., Wright, E. L., Conrow, T., et al. 2013, Explanatory Supplement to the AllWISE Data Release Products, Tech. Rep. 1

Day-Jones, A. C., Pinfield, D. J., Ruiz, M. T., et al. 2011, MNRAS, 410, 705 Debes, J. H., Thévenot, M., Kuchner, M. J., et al. 2019, ApJL, 872, L25

Dey, A., Schlegel, D. J., Lang, D., et al. 2019, AJ, 157, 168

Dotter, A. 2016, ApJS, 222, 8

Dupuy, T. J., \& Liu, M. C. 2012, ApJS, 201, 19

Dye, S., Lawrence, A., Read, M. A., et al. 2018, MNRAS, 473, 5113 Edge, A., Sutherland, W., Kuijken, K., et al. 2013, Msngr, 154, 32

Eisenhardt, P. R. M., Marocco, F., Fowler, J. W., et al. 2020, ApJS, 247, 69 Faherty, J. K., Burgasser, A. J., Cruz, K. L., et al. 2009, AJ, 137, 1

Faherty, J. K., Goodman, S., Caselden, D., et al. 2020, ApJ, 889, 176

Fazio, G. G., Hora, J. L., Allen, L. E., et al. 2004, ApJS, 154, 10

Fitzpatrick, M. J., Olsen, K., Economou, F., et al. 2014, Proc. SPIE, 9149, $91491 \mathrm{~T}$

Fontaine, G., Brassard, P., \& Bergeron, P. 2001, PASP, 113, 409

Gaia Collaboration, Brown, A. G. A., Vallenari, A., et al. 2018, A\&A, 616, A1 Gaia Collaboration, Prusti, T., de Bruijne, J. H. J., et al. 2016, A\&A, 595, A1 Gardner, J. P., Mather, J. C., Clampin, M., et al. 2006, SSRv, 123, 485

Gentile Fusillo, N. P., Tremblay, P.-E., Gänsicke, B. T., et al. 2019, MNRAS, 482,4570

Greisen, E. W., \& Calabretta, M. R. 2002, A\&A, 395, 1061

Hambly, N. C., Collins, R. S., Cross, N. J. G., et al. 2008, MNRAS, 384, 637 Hawley, S. L., Covey, K. R., Knapp, G. R., et al. 2002, AJ, 123, 3409

Kirkpatrick, J. D., Cushing, M. C., Gelino, C. R., et al. 2011, ApJS, 197, 19 Kirkpatrick, J. D., Gelino, C. R., Cushing, M. C., et al. 2012, ApJ, 753, 156 Kirkpatrick, J. D., Kellogg, K., Schneider, A. C., et al. 2016, ApJS, 224, 36 Kirkpatrick, J. D., Martin, E. C., Smart, R. L., et al. 2019, ApJS, 240, 19 Kirkpatrick, J. D., Schneider, A., Fajardo-Acosta, S., et al. 2014, ApJ, 783, 122 Kuchner, M. J., Faherty, J. K., Schneider, A. C., et al. 2017, ApJL, 841, L19 Lang, D., Hogg, D. W., Mierle, K., Blanton, M., \& Roweis, S. 2010, AJ, 139,1782

Lawrence, A., Warren, S. J., Almaini, O., et al. 2007, MNRAS, 379, 1599 Limoges, M. M., Bergeron, P., \& Lépine, S. 2015, ApJS, 219, 19

Limoges, M. M., Lépine, S., \& Bergeron, P. 2013, AJ, 145, 136

Lindegren, L., Hernández, J., Bombrun, A., et al. 2018, A\&A, 616, A2

Luhman, K. L. 2013, ApJL, 767, L1

Luhman, K. L. 2014a, ApJ, 781, 4

Luhman, K. L. 2014b, ApJL, 786, L18

Luhman, K. L., Burgasser, A. J., \& Bochanski, J. J. 2011, ApJL, 730, L9

Luhman, K. L., Morley, C. V., Burgasser, A. J., Esplin, T. L., \& Bochanski, J. J. 2014, ApJ, 794, 16

Mace, G. N., Kirkpatrick, J. D., Cushing, M. C., et al. 2013, ApJ, 777, 36

Mainzer, A., Bauer, J., Cutri, R., et al. 2014, ApJ, 792, 30

Mainzer, A., Bauer, J., Grav, T., et al. 2011a, ApJ, 731, 53

Mainzer, A., Cushing, M. C., Skrutskie, M., et al. 2011b, ApJ, 726, 30

Makovoz, D., \& Khan, I. 2005, in ASP Conf. Ser. 347, Astronomical Data Analysis Software and Systems XIV, ed. P. Shopbell, M. Britton, \& R. Ebert (San Francisco, CA: ASP), 81

Makovoz, D., \& Marleau, F. R. 2005, PASP, 117, 1113

Marocco, F., Caselden, D., Meisner, A. M., et al. 2019, ApJ, 881, 17

Marocco, F., Kirkpatrick, J. D., Meisner, A. M., et al. 2020, ApJL, 888, L19

Marsh, T. R. 1995, MNRAS, 275, L1

Martin, E. C., Kirkpatrick, J. D., Beichman, C. A., et al. 2018, ApJ, 867, 109

Matese, J. J., Whitman, P. G., \& Whitmire, D. P. 1999, Icar, 141, 354

Matese, J. J., \& Whitmire, D. P. 2011, Icar, 211, 926

McMahon, R. G., Banerji, M., Gonzalez, E., et al. 2013, Msngr, 154, 35

Meisner, A., Bromley, B. C., Nugent, P. E., et al. 2017, AJ, 153, 65 
Meisner, A. M., Bromley, B. C., Kenyon, S. J., \& Anderson, T. E. 2018a, AJ, 155,166

Meisner, A. M., Caselden, D., Kirkpatrick, J. D., et al. 2020, ApJ, 889, 74

Meisner, A. M., Lang, D., Schlafly, E. F., \& Schlegel, D. J. 2019, PASP, 131, 124504

Meisner, A. M., Lang, D., \& Schlegel, D. J. 2018b, AJ, 156, 69

Meisner, A. M., Lang, D. A., \& Schlegel, D. J. 2018c, RNAAS, 2, 202

Morley, C. V., Skemer, A. J., Allers, K. N., et al. 2018, ApJ, 858, 97

Nidever, D. L., Dey, A., Olsen, K., et al. 2018, AJ, 156, 131

Ochsenbein, F., Bauer, P., \& Marcout, J. 2000, A\&AS, 143, 23

Patten, B. M., Stauffer, J. R., Burrows, A., et al. 2006, ApJ, 651, 502

Paxton, B., Bildsten, L., Dotter, A., et al. 2011, ApJS, 192, 3

Racine, R. 1978, JRASC, 72, 324

Saumon, D., \& Marley, M. S. 2008, ApJ, 689, 1327

Schlafly, E. F., Green, G. M., Lang, D., et al. 2018, ApJS, 234, 39

Schlafly, E. F., Meisner, A. M., \& Green, G. M. 2019, ApJS, 240, 30

Schneider, A. C., Cushing, M. C., Kirkpatrick, J. D., et al. 2015, ApJ, 804, 92

Schneider, A. C., Greco, J., Cushing, M. C., et al. 2016, ApJ, 817, 112
Scholz, R. D. 2014, A\&A, 561, A113

Simcoe, R. A., Burgasser, A. J., Schechter, P. L., et al. 2013, PASP, 125, 270

Simpson, R., Page, K. R., \& De Roure, D. 2014, in Proc. of the 23rd Int. Conf. on World Wide Web, WWW 14 Companion (New York: Association for Computing Machinery), 1049

Skemer, A. J., Morley, C. V., Allers, K. N., et al. 2016, ApJL, 826, L17

Skrutskie, M. F., Cutri, R. M., Stiening, R., et al. 2006, AJ, 131, 1163

Vacca, W. D., Cushing, M. C., \& Rayner, J. T. 2003, PASP, 115, 389

Wenger, M., Ochsenbein, F., Egret, D., et al. 2000, A\&AS, 143, 9

Werner, M. W., Roellig, T. L., Low, F. J., et al. 2004, ApJS, 154, 1

Wilson, J. C., Henderson, C. P., Herter, T. L., et al. 2004, Proc. SPIE, 5492, 1295

Wright, E., Eisenhardt, P., Mainzer, A., et al. 2010, AJ, 140, 1868

Wright, E. L., Mainzer, A., Kirkpatrick, J. D., et al. 2014, AJ, 148, 82

Zhang, Z., Liu, M. C., Hermes, J. J., et al. 2020, ApJ, 891, 171

Zhang, Z. H., Burgasser, A. J., Gálvez-Ortiz, M. C., et al. 2019, MNRAS, 486,1260 